Supporting Information

\title{
A Series of Tetravalent Actinide Amidinates: Structure Determination and Bonding Analysis.
}

Roger Kloditz ${ }^{1 \ddagger}$, Sebastian Fichter ${ }^{1 \ddagger}$, Sebastian Kaufmann ${ }^{2}$, Tobias S. Brunner ${ }^{2}$, Peter Kaden ${ }^{1}$, Michael Patzschke ${ }^{1}$, Thorsten Stumpf ${ }^{1}$, Peter W. Roesky ${ }^{2}$, Moritz Schmidt, ${ }^{1}$ and Juliane März ${ }^{*} 1$

${ }^{1}$ Helmholtz-Zentrum Dresden-Rossendorf, Institute of Resource Ecology, Bautzner Landstraße 400, 01328 Dresden, Germany.

${ }^{2}$ Karlsruhe Institute of Technology, Institute for Inorganic Chemistry, Engesserstraße 15, 76131 Karlsruhe, Germany.

(* Corresponding author: Juliane März, ${ }^{\star}$ Both authors contributed equally) 


\section{Table of contents}

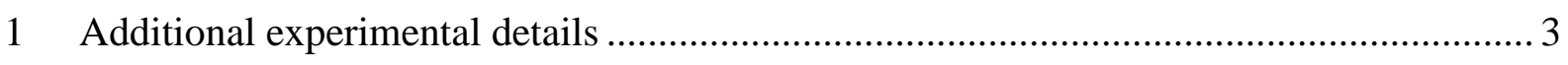

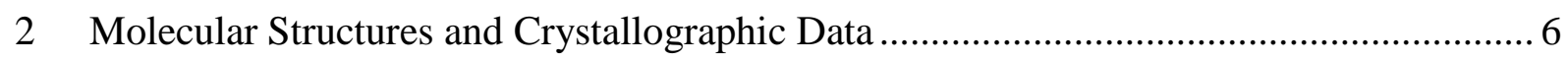

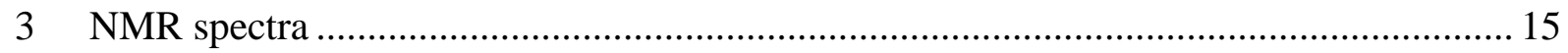

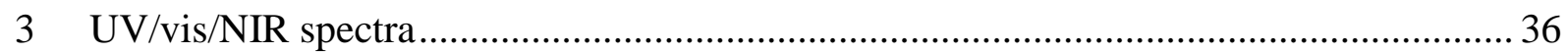

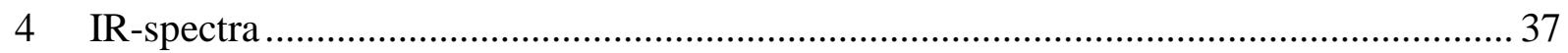

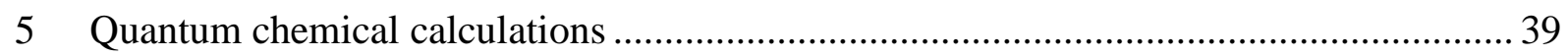

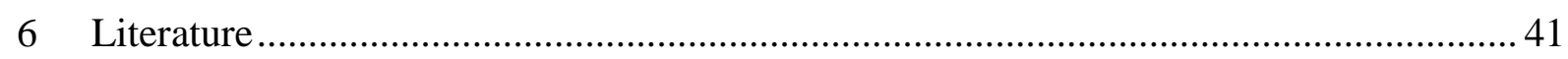




\section{Additional experimental details}

Attempted synthesis of Ce(IV) complex starting from [NEt $]_{2}\left[\mathrm{CeCl}_{6}\right]$

[NEt 4$]\left[\mathrm{CeCl}_{2}((\mathrm{~S})-\mathrm{PEBA})_{2}\right]$ A $62.4 \mathrm{mg}\left(0.102 \mathrm{mmol}, 1\right.$ eq.) $\left[\mathrm{NEt}_{4}\right]_{2}\left[\mathrm{CeCl}_{6}\right]$ were suspended in $1 \mathrm{~mL}$ of thf. A solution containing $66.5 \mathrm{mg}(0.202 \mathrm{mmol}, 2$ eq.) $(S)$-HPEBA and $47.5 \mathrm{mg}$ (0.284 mmol, 2.8 eq.) LiHMDS in $1 \mathrm{~mL}$ of thf was slowly added and the suspension was stirred for $16 \mathrm{~h}$. The supernatant was slowly evaporated after centrifugation yielding pale-yellow crystals.

Yield: $\quad 42.0 \mathrm{mg}$ pale-yellow crystals $(42 \%)$

$\mathrm{C}_{54} \mathrm{H}_{66} \mathrm{~N}_{5} \mathrm{Cl}_{2} \mathrm{Ce} \quad 996.14 \mathrm{~g} / \mathrm{mol}$

NMR:

${ }^{1} \mathrm{H}:(400 \mathrm{MHz}$, toluene-d8, 298K) $\delta[\mathrm{ppm}]: 11.47$ (s, 4H, NCH), 10.94 (s, $\left.4 \mathrm{H}, o-\mathrm{PhH}^{\mathrm{NCN}}\right), 8.73\left(\mathrm{~s}, 4 \mathrm{H}, m-\mathrm{PhH}^{\mathrm{NCN}}\right), 8.42\left(\mathrm{~s}, 2 \mathrm{H}, p-\mathrm{PhH}^{\mathrm{NCN}}\right), 6.06$ (s, 4H, $p-\mathrm{PhH}), 5.57$ (s, 8H, m-PhH), 2.93 (s, 8H, o-PhH), -0.69 (s, 12H, $\mathrm{Me})$.

${ }^{13} \mathrm{C}\left\{{ }^{1} \mathrm{H}\right\}:(100.58 \mathrm{MHz}$, toluene-d $8,298 \mathrm{~K}): 151.3(i-\mathrm{Ph}), 148.5\left(i-\mathrm{Ph}^{\mathrm{NCN}}\right)$, $132.0\left(o-\mathrm{Ph}^{\mathrm{NCN}}\right), 130.3\left(m-\mathrm{Ph}^{\mathrm{NCN}}\right), 129.6\left(p-\mathrm{Ph}^{\mathrm{NCN}}\right), 126.7(m-\mathrm{Ph}), 125.1$ $(o-\mathrm{Ph}), 124.35(p-\mathrm{Ph}), 68.0(\mathrm{NCH}), 26.7(\mathrm{Me})$, the signal of $\mathrm{NCN}$ could not be observed.

IR: $\quad 701(\mathrm{vs}), 740(\mathrm{~s}), 771(\mathrm{~s}), 786(\mathrm{~m}), 796(\mathrm{~s}), 796(\mathrm{~s}), 1004(\mathrm{~s}), 1022(\mathrm{~m})$, 1027 (m), 1066 (m), 1080 (m), 1090 (w), 1174 (m), 1184 (m), 1201 (w), 1301 (w), 1321 (m), 1331 (m), 1351 (m), 1393 (s), 1457 (vs), 1487 (m), $2964(w)$.

SC-XRD: $\quad$ CCDC 1936875; orthorhombic $P 2{ }_{1} 2_{1} 2_{1} ; \quad a=12.514(1) \AA$, $\mathrm{b}=17.275(1) \AA, \quad \mathrm{c}=23.085(2) \AA ; \quad \mathrm{V}=4990.6(5) \AA^{3} ; 100 \mathrm{~K} ; \mathrm{Z}=4$; $1.326 \mathrm{~g} / \mathrm{cm}^{3} ; 1.059 \mathrm{~mm}^{-1} ; \quad \theta_{\max }=26.02^{\circ} ; \mathrm{R} 1=0.032 ; \quad$ wR2 $=0.062 ;$ $\mathrm{R}_{\text {int }}=0.064 ; \mathrm{GooF}=1.045 ;$ Flack $\mathrm{x}=0.009(6)$. 


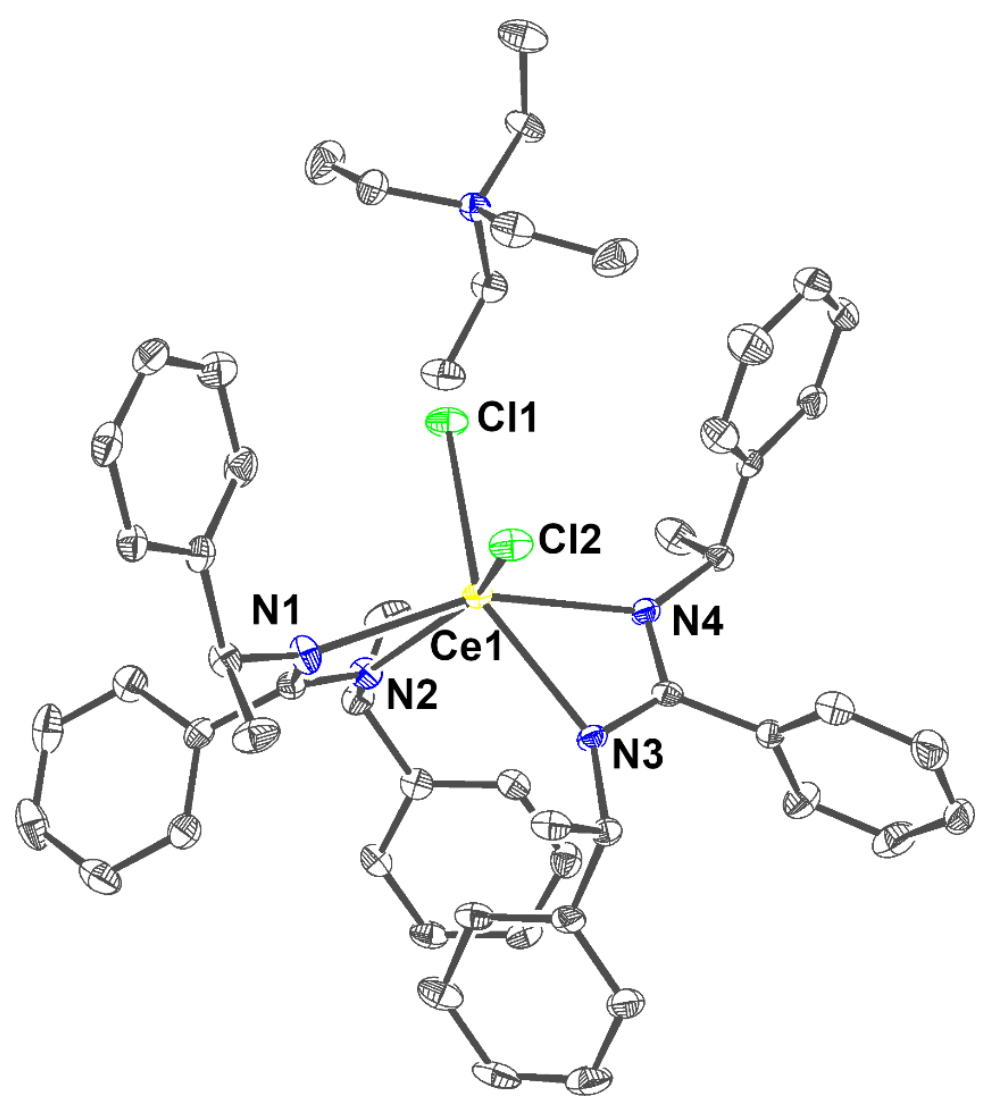

Figure S1. Molecular structure of $\left[\mathrm{NEt}_{4}\right]\left[\mathrm{CeCl}_{2}((S) \text {-PEBA })_{2}\right]$. Ellipsoids are drawn at $50 \%$ probability level. Hydrogen atoms are omitted for clarity. Colour code: carbon ( $\mathrm{C}$, dark gray), chlorine ( $\mathrm{Cl}$, light green), nitrogen $(\mathrm{N}$, blue), and cerium (Ce, yellow). 


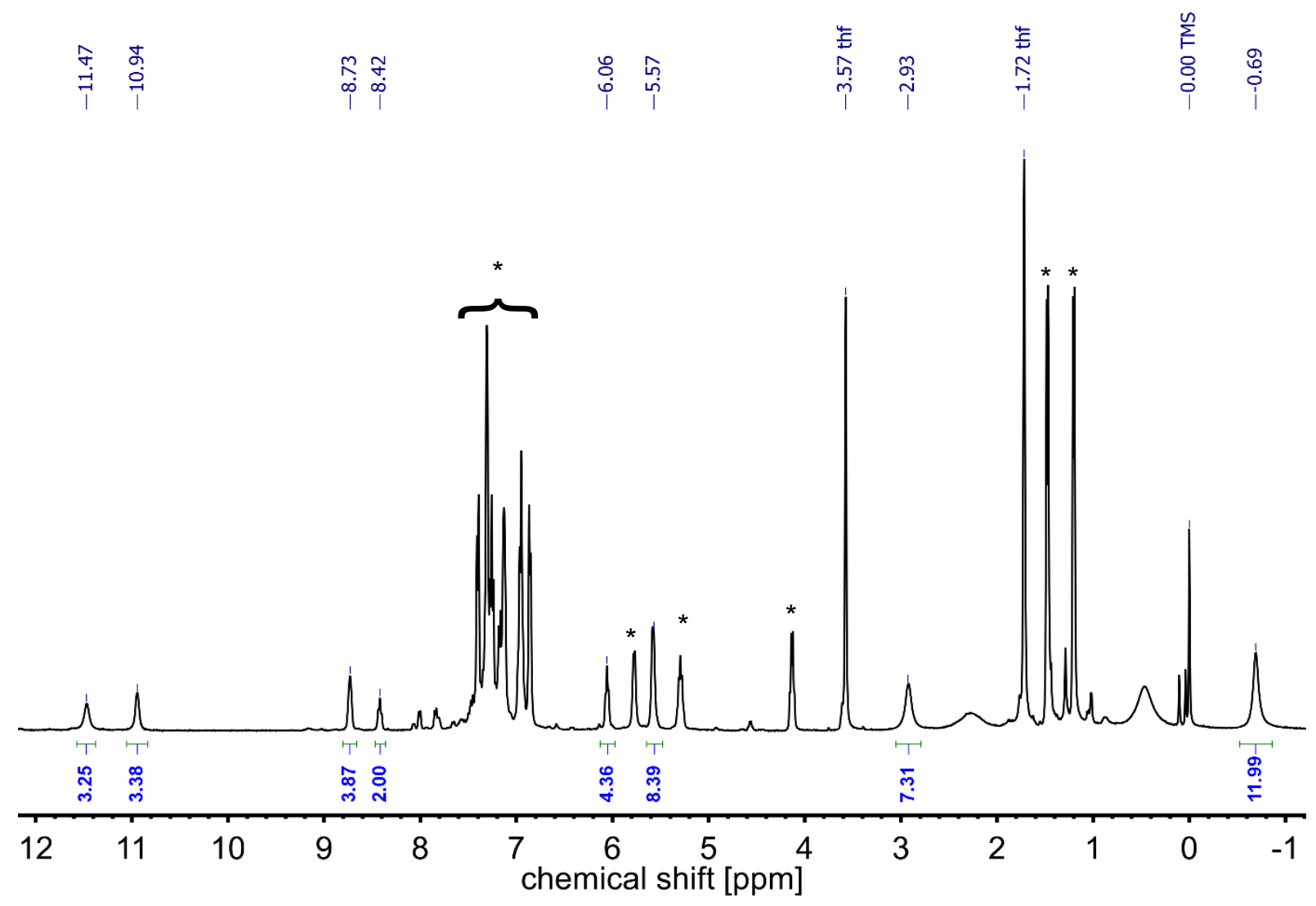

Figure S2. ${ }^{1} \mathrm{H}-\mathrm{NMR}$ spectrum of residue from attempted synthesis of $\mathrm{Ce}(\mathrm{IV})$ complex indicating a mixture of $\left[\mathrm{NEt}_{4}\right]\left[\mathrm{CeCl}_{2}((S)-\mathrm{PEBA})_{2}\right]$ (blue) and $(S)$-HPEBA (marked with asterisk) in thf-d $\mathrm{d}_{8}$ at $298 \mathrm{~K}$.

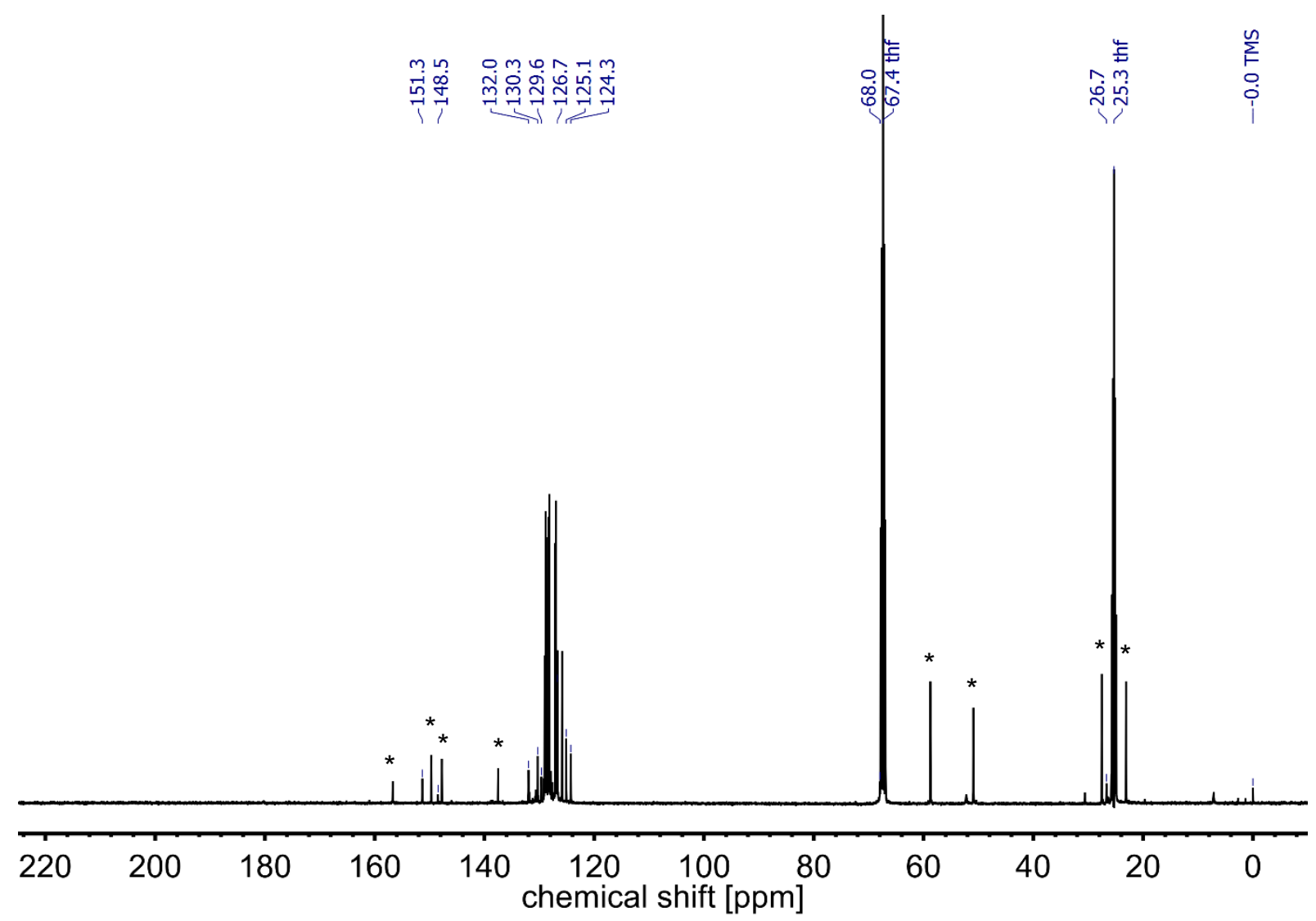

Figure S3. ${ }^{13} \mathrm{C}$-NMR spectrum of residue from attempted synthesis of $\mathrm{Ce}(\mathrm{IV})$ complex indicating a mixture of $\left[\mathrm{NEt}_{4}\right]\left[\mathrm{CeCl}_{2}((S)-\mathrm{PEBA})_{2}\right]$ (blue) and (S)-HPEBA (marked with asterisk) in thf-d $\mathrm{d}_{8}$ at $298 \mathrm{~K}$. 


\section{Molecular Structures and Crystallographic Data}

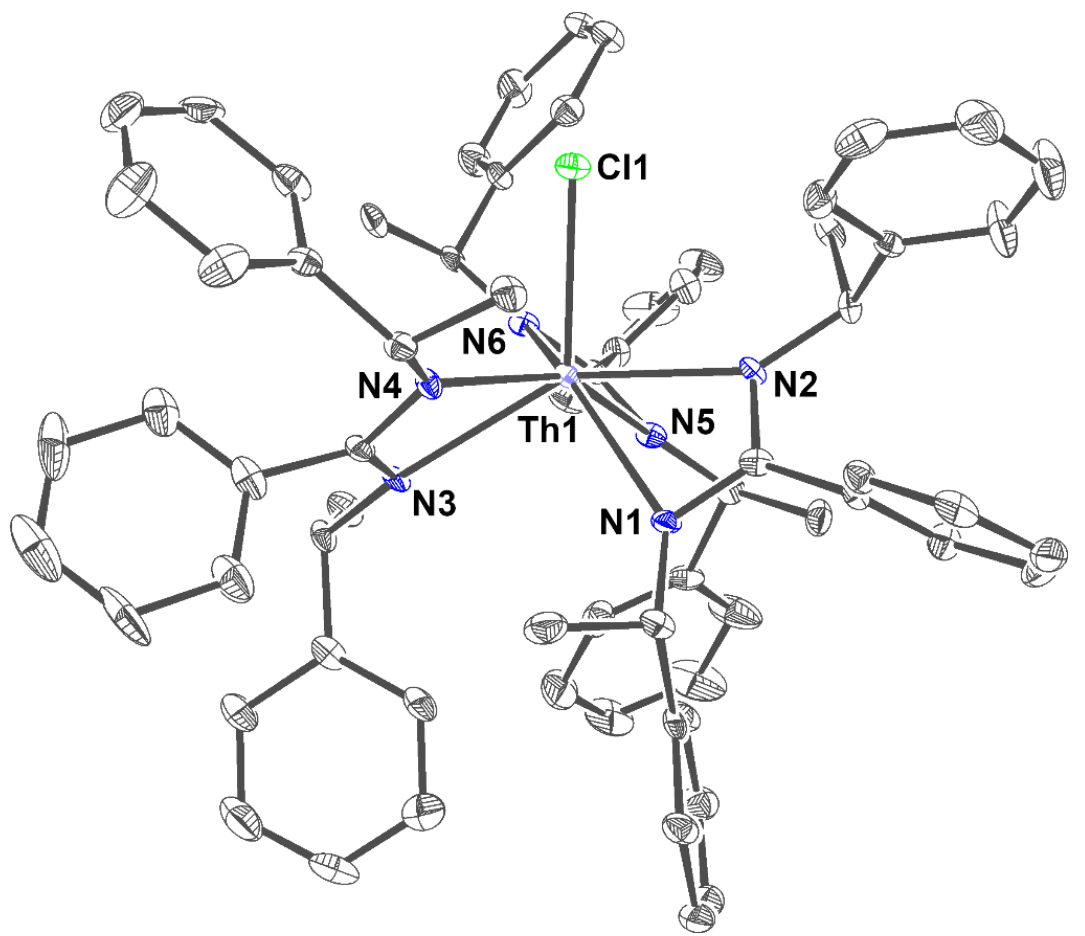

Figure S4. Molecular structure of $\left[\mathrm{ThCl}((S)-\mathrm{PEBA})_{3}\right](\mathbf{1})$. Ellipsoids are drawn at $50 \%$ probability level. Hydrogen atoms and solvent molecules are omitted for clarity. Colour code: carbon (C, dark gray), chlorine $(\mathrm{Cl}$, light green), nitrogen $(\mathrm{N}$, blue), and thorium (Th, pale blue).

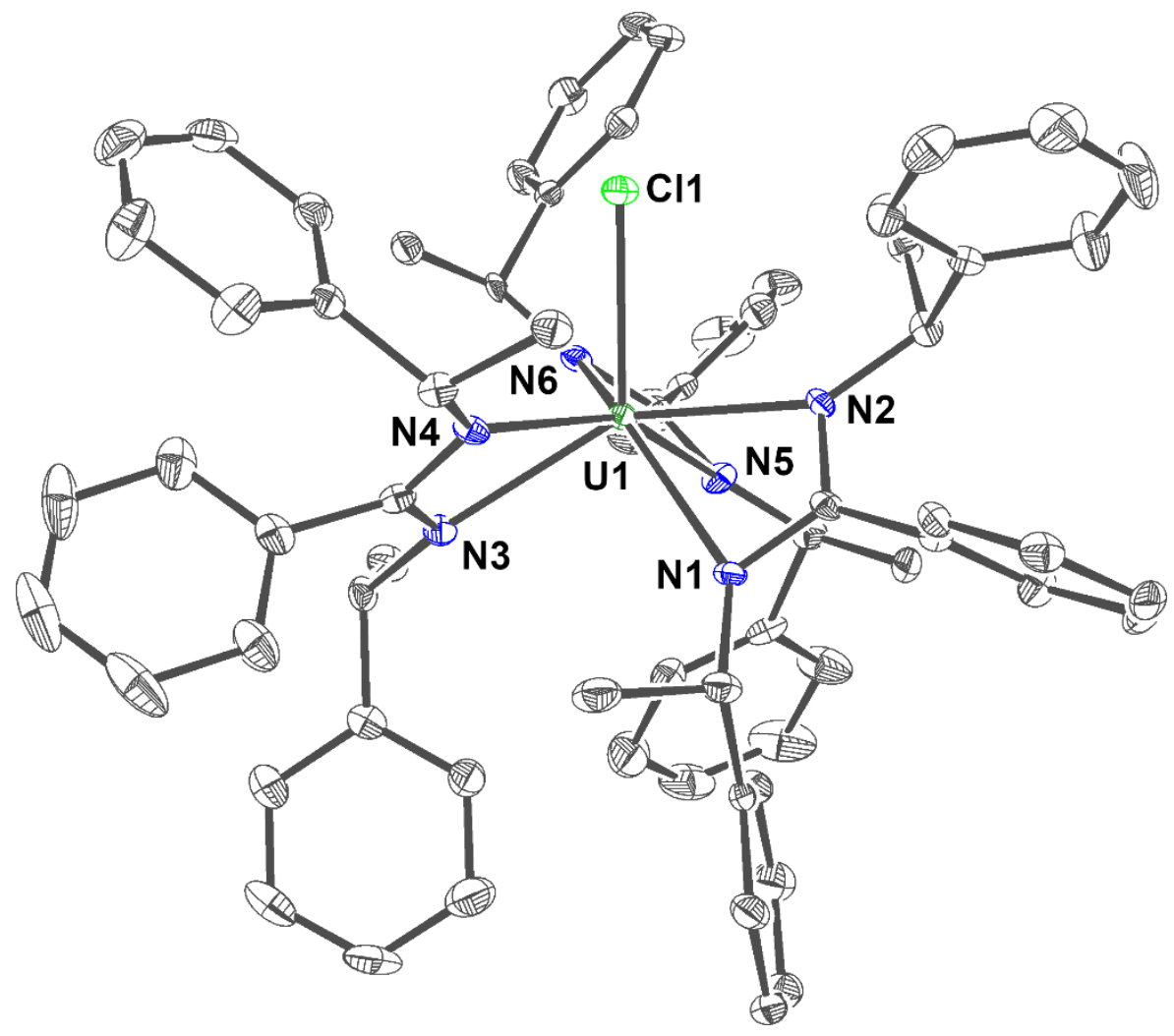

Figure S5. Molecular structure of $\left[\mathrm{UCl}((S)-\mathrm{PEBA})_{3}\right](2)$. Ellipsoids are drawn at $50 \%$ probability level. Hydrogen atoms and solvent molecules are omitted for clarity. Colour code: carbon $(\mathrm{C}$, dark gray), chlorine $(\mathrm{Cl}$, light green), nitrogen (N, blue), and uranium (U, dark green). 


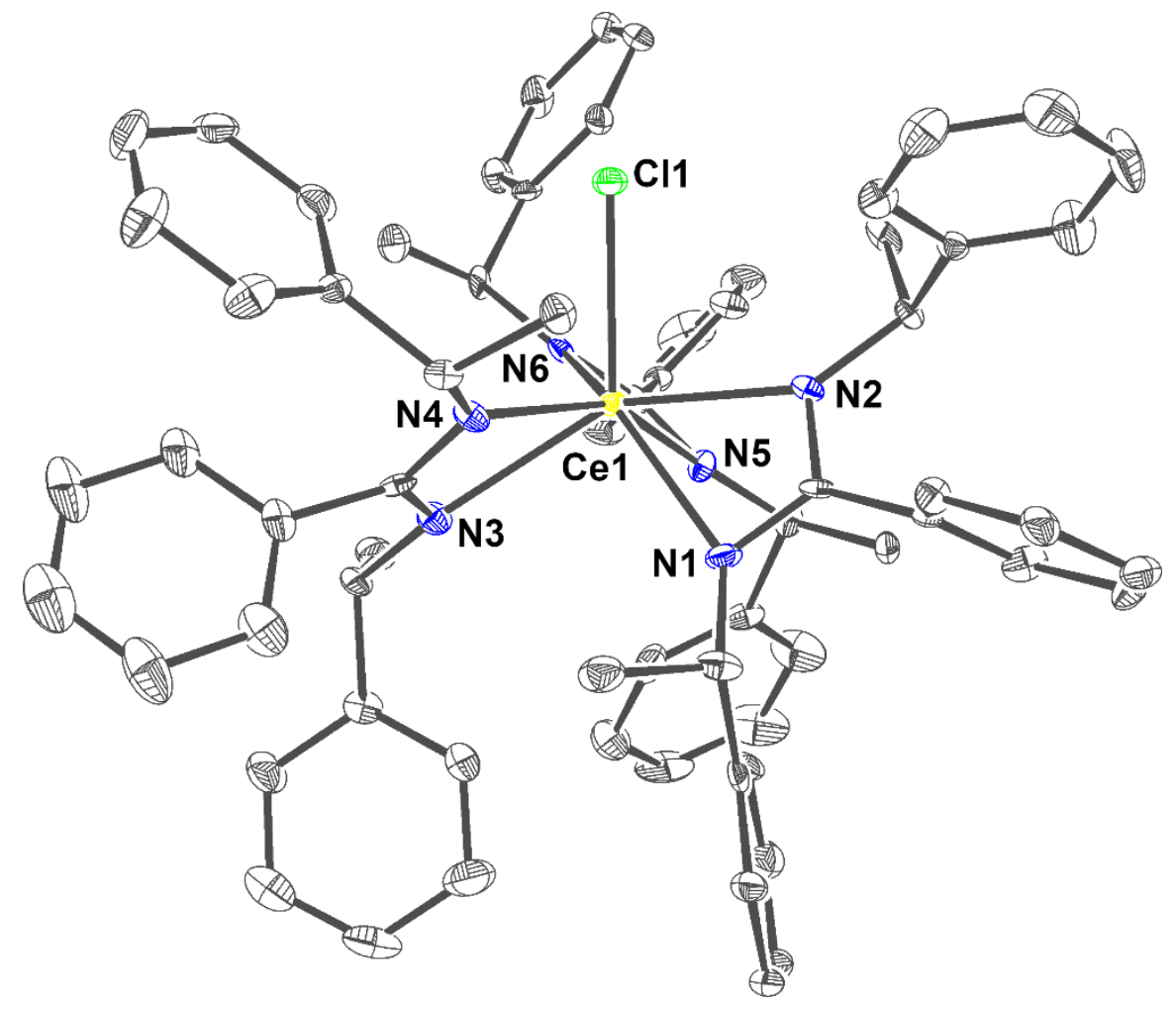

Figure S6. Molecular structure of $\left[\mathrm{CeCl}((S)-\mathrm{PEBA})_{3}\right](4)$. Ellipsoids are drawn at $50 \%$ probability level. Hydrogen atoms and solvent molecules are omitted for clarity. Colour code: carbon (C, dark gray), chlorine $(\mathrm{Cl}$, light green), nitrogen $(\mathrm{N}$, blue $)$, and cerium ( $\mathrm{Ce}$, yellow).

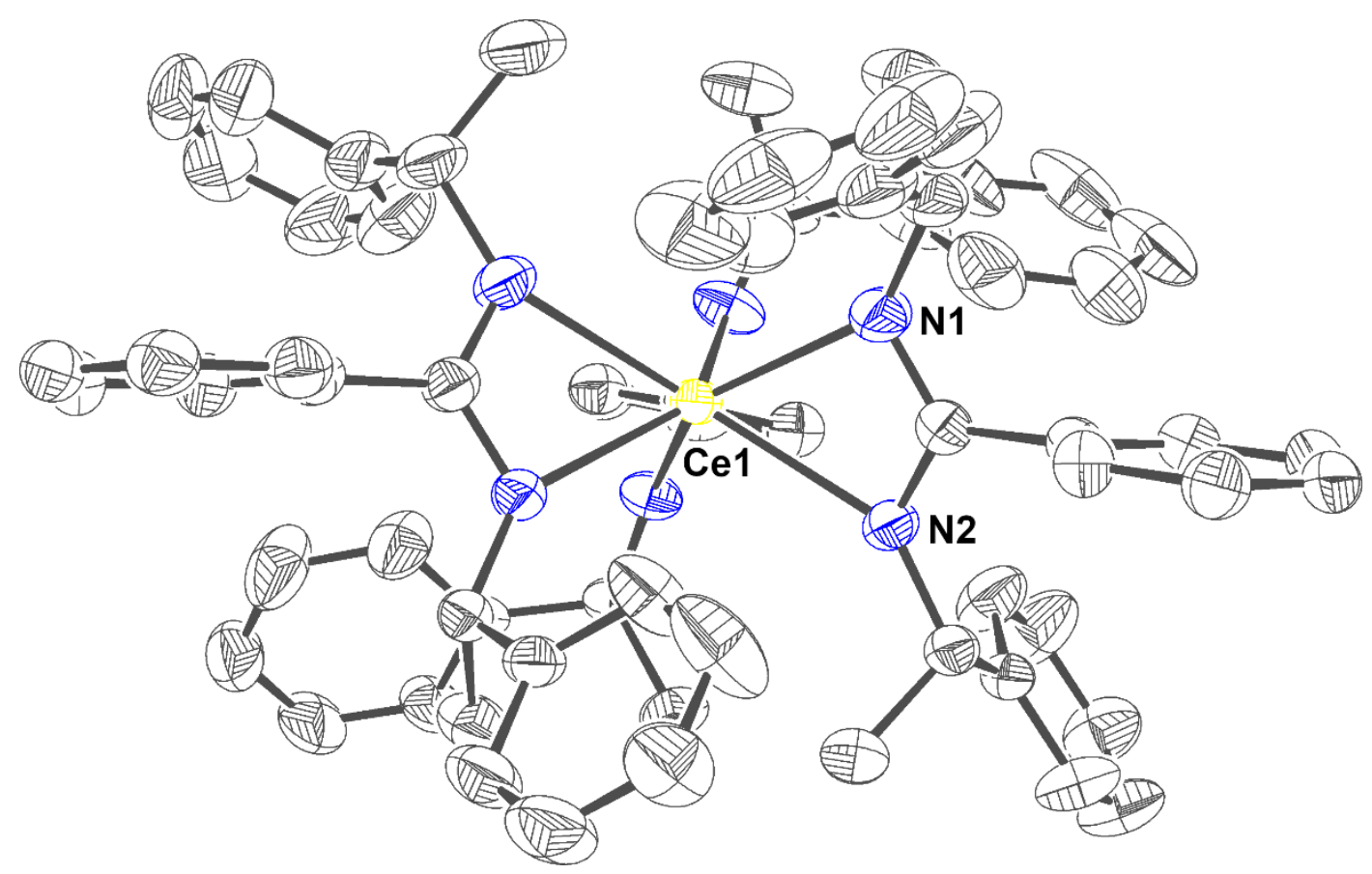

Figure S7. Molecular structure of $\left[\mathrm{Ce}((S)-\mathrm{PEBA})_{3}\right](5)$. Ellipsoids are drawn at $50 \%$ probability level. Hydrogen atoms are omitted for clarity. Colour code: carbon $(\mathrm{C}$, dark gray), chlorine ( $\mathrm{Cl}$, light green), nitrogen (N, blue), and cerium (Ce, yellow). 


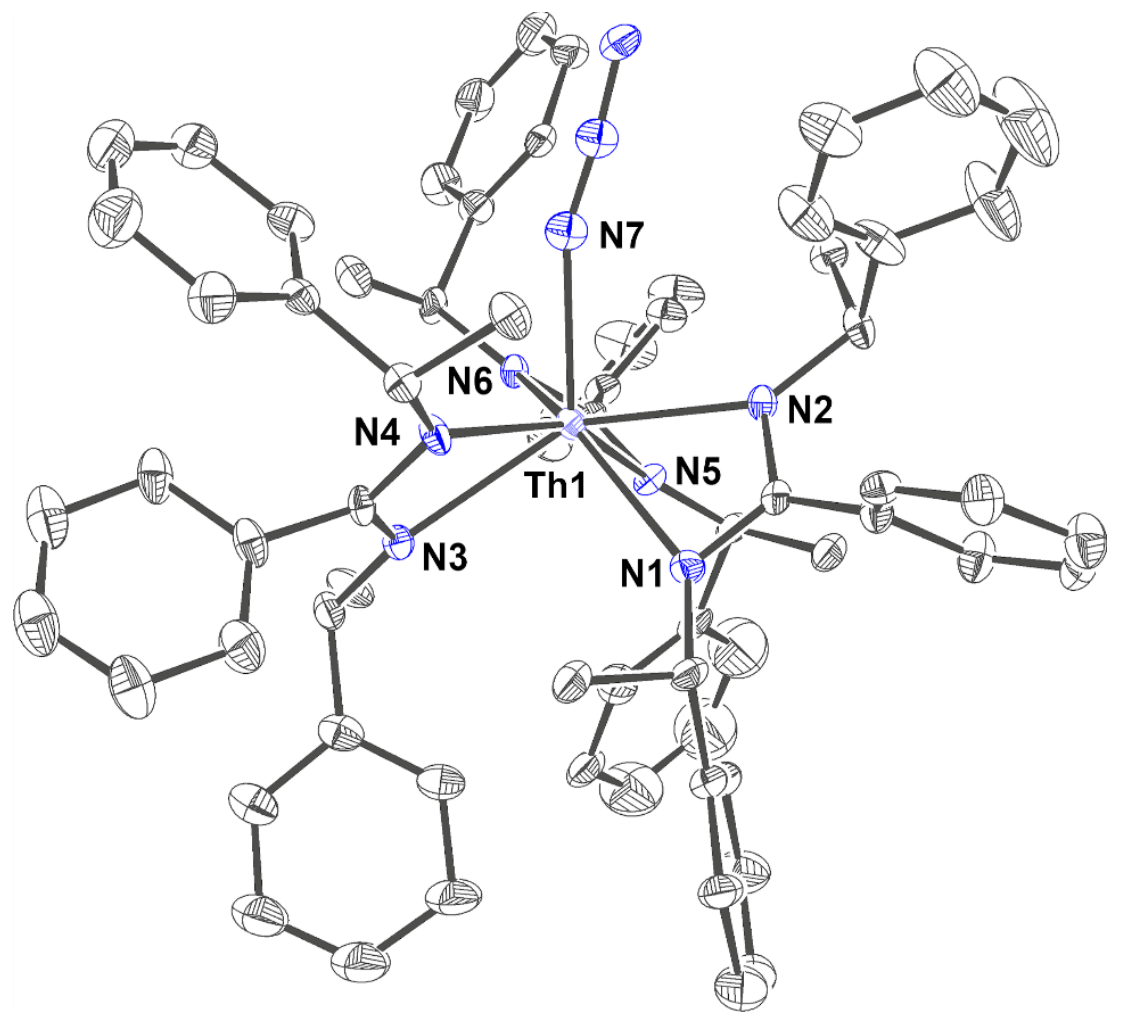

Figure S8. Molecular structure of $\left[\mathrm{ThN}_{3}((S) \text {-PEBA })_{3}\right](\mathbf{6})$. Ellipsoids are drawn at $50 \%$ probability level. Hydrogen atoms and solvent molecules are omitted for clarity. Colour code: carbon (C, dark gray), nitrogen ( $\mathrm{N}$, blue), and thorium (Th, pale blue).

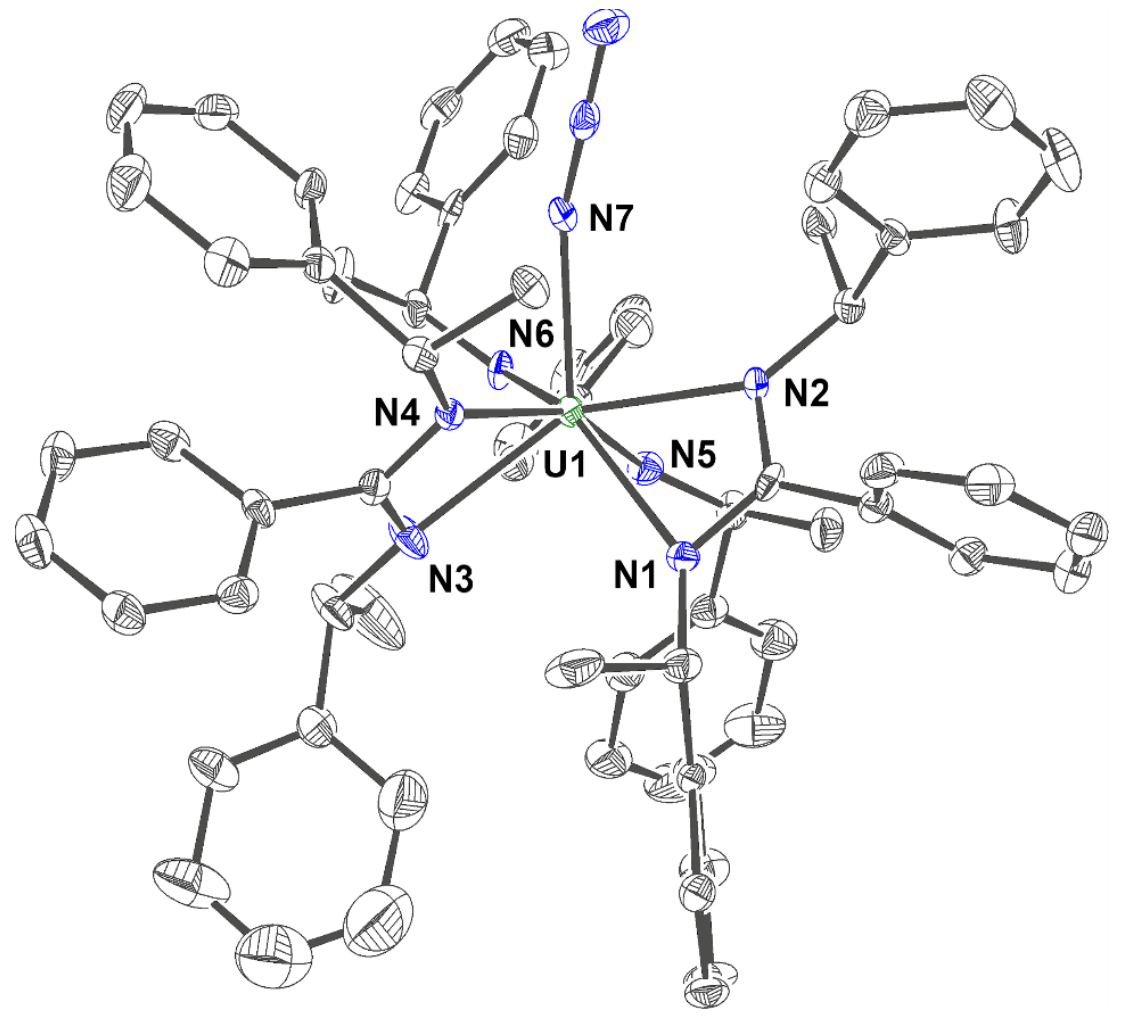

Figure S9. Molecular structure of $\left[\mathrm{UN}_{3}((S)-\mathrm{PEBA})_{3}\right](7)$. Ellipsoids are drawn at $50 \%$ probability level. Hydrogen atoms and solvent molecules are omitted for clarity. Colour code: carbon $(\mathrm{C}$, dark gray), nitrogen $(\mathrm{N}$, blue), and uranium (U, green). 
Table S1. Intramolecular distances between metal centre $\mathrm{M}$ and coordinating atoms $\mathrm{X}$ in isostructural $\left[\mathrm{MCl}((S)-\mathrm{PEBA})_{3}\right]$ complexes $\mathbf{1}, \mathbf{2}$, and $\mathbf{4}$.

\begin{tabular}{llll}
\hline $\mathrm{d}(\mathrm{M}-\mathrm{X})[\AA]$ & $\mathbf{1}(\mathrm{M}=\mathrm{Th})$ & $\mathbf{2}(\mathrm{M}=\mathrm{U})$ & $\mathbf{4}(\mathrm{M}=\mathrm{Ce})$ \\
\hline $\mathrm{C} 11$ & $2.701(2)$ & $2.641(1)$ & $2.644(2)$ \\
$\mathrm{N} 1$ & $2.548(5)$ & $2.495(4)$ & $2.507(5)$ \\
$\mathrm{N} 2$ & $2.439(5)$ & $2.372(4)$ & $2.362(5)$ \\
$\mathrm{N} 3$ & $2.555(5)$ & $2.497(4)$ & $2.508(6)$ \\
$\mathrm{N} 4$ & $2.456(5)$ & $2.406(4)$ & $2.392(5)$ \\
$\mathrm{N} 5$ & $2.577(6)$ & $2.505(4)$ & $2.521(6)$ \\
$\mathrm{N} 6$ & $2.423(6)$ & $2.360(4)$ & $2.341(5)$ \\
\hline
\end{tabular}

Table S2. Intramolecular distances between metal centre $M$ and coordinating nitrogen atoms N1-N7 in isostructural $\left[\mathrm{AnN}_{3}((S) \text {-PEBA })_{3}\right]$ complexes 6 and 7.

\begin{tabular}{lll}
\hline $\mathrm{d}(\mathrm{M}-\mathrm{X})[\AA]$ & $\mathbf{6}(\mathrm{M}=\mathrm{Th})$ & $\mathbf{7}(\mathrm{M}=\mathrm{U})$ \\
\hline $\mathrm{N} 1$ & $2.556(15)$ & $2.483(6)$ \\
$\mathrm{N} 2$ & $2.427(15)$ & $2.375(6)$ \\
$\mathrm{N} 3$ & $2.566(17)$ & $2.488(6)$ \\
$\mathrm{N} 4$ & $2.456(16)$ & $2.394(5)$ \\
$\mathrm{N} 5$ & $2.559(17)$ & $2.511(7)$ \\
$\mathrm{N} 6$ & $2.407(16)$ & $2.397(6)$ \\
$\mathrm{N} 7$ & $2.342(16)$ & $2.293(6)$ \\
\hline
\end{tabular}

Table S3. Angles $\xi$ of plane normals of N1-M1-N2 (A), N3-M1-N4 (B) and N5-M1-N6 (C) against M1-Cl1 bond. See Figure S13.

\begin{tabular}{llll}
\hline & $\mathbf{1}(\mathrm{M}=\mathrm{Th})$ & $\mathbf{2}(\mathrm{M}=\mathrm{U})$ & $\mathbf{4}(\mathrm{M}=\mathrm{Ce})$ \\
\hline A & $53.0^{\circ}$ & $53.3^{\circ}$ & $53.4^{\circ}$ \\
B & $47.9^{\circ}$ & $46.5^{\circ}$ & 46.6 \\
C & $40.1^{\circ}$ & $40.7^{\circ}$ & 40.9 \\
\hline & $\mathbf{6}(\mathrm{M}=\mathrm{Th})$ & $\mathbf{7}(\mathrm{M}=\mathrm{U})$ & \\
\hline A & $52.7^{\circ}$ & $57.0^{\circ}$ & \\
B & $47.5^{\circ}$ & $47.7^{\circ}$ & \\
C & $40.2^{\circ}$ & $35.9^{\circ}$ & \\
\hline
\end{tabular}

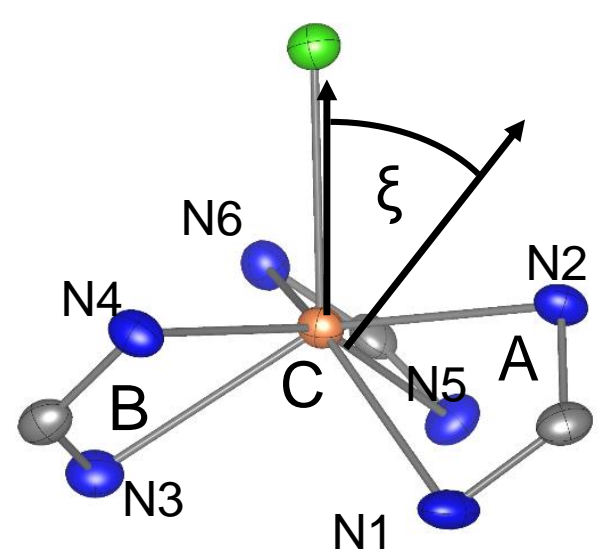

Figure S10. Graphical representation of angle between plane normal c (N5-M1-N6) against M1-Cl1 bond. 
Table S4. Intramolecular distances within amidinate moieties in isostructural $\left[\mathrm{MCl}((S)-\mathrm{PEBA})_{3}\right]$ complexes $\mathbf{1}, \mathbf{2}$, and 4.

\begin{tabular}{llll}
\hline $\mathrm{d}[\AA]$ & $\mathbf{1}(\mathrm{M}=\mathrm{Th})$ & $\mathbf{2}(\mathrm{M}=\mathrm{U})$ & $\mathbf{4}(\mathrm{M}=\mathrm{Ce})$ \\
\hline $\mathrm{N} 1-\mathrm{C} 9$ & $1.345(8)$ & $1.335(6)$ & $1.337(8)$ \\
$\mathrm{N} 2-\mathrm{C} 9$ & $1.338(8)$ & $1.351(6)$ & $1.358(8)$ \\
$\mathrm{N} 3-\mathrm{C} 32$ & $1.329(9)$ & $1.328(7)$ & $1.333(9)$ \\
$\mathrm{N} 4-\mathrm{C} 32$ & $1.360(8)$ & $1.345(7)$ & $1.341(9)$ \\
$\mathrm{N} 5-\mathrm{C} 55$ & $1.341(9)$ & $1.341(6)$ & $1.337(8)$ \\
$\mathrm{N} 6-\mathrm{C} 55$ & $1.321(9)$ & $1.330(6)$ & $1.331(8)$ \\
\hline
\end{tabular}

Table S5. Intramolecular distances within amidinate moieties in isostructural $\left[\mathrm{MN}_{3}((S)-\mathrm{PEBA})_{3}\right]$ complexes 6 and 7.

\begin{tabular}{lll}
\hline $\mathrm{d}[\AA]$ & $\mathbf{6}(\mathrm{M}=\mathrm{Th})$ & $\mathbf{7}(\mathrm{M}=\mathrm{U})$ \\
\hline $\mathrm{N} 1-\mathrm{C} 9$ & $1.34(3)$ & $1.343(10)$ \\
$\mathrm{N} 2-\mathrm{C} 9$ & $1.36(3)$ & $1.343(9)$ \\
$\mathrm{N} 3-\mathrm{C} 32$ & $1.35(3)$ & $1.330(10)$ \\
$\mathrm{N} 4-\mathrm{C} 32$ & $1.36(3)$ & $1.345(10)$ \\
$\mathrm{N} 5-\mathrm{C} 55$ & $1.29(3)$ & $1.332(10)$ \\
$\mathrm{N} 6-\mathrm{C} 55$ & $1.33(3)$ & $1.327(10)$ \\
\hline
\end{tabular}

Table S6. Comparison of intramolecular distances of compounds $\mathbf{1}$ and $\mathbf{2}$ with literature data of tetravalent actinide tris(amidinate) complexes. Bond lengths $\mathrm{M}-\mathrm{N} 1, \mathrm{M}-\mathrm{N} 3, \mathrm{M}-\mathrm{N} 5$ were averaged to $\mathrm{M}-\mathrm{N}_{\mathrm{a}}$ and distances $\mathrm{M}-\mathrm{N} 2, \mathrm{M}-\mathrm{N} 4, \mathrm{M}-\mathrm{N} 6$ to $\mathrm{M}-\mathrm{N}_{\mathrm{b}}$.

\begin{tabular}{llll}
\hline $\mathrm{d}(\mathrm{M}-\mathrm{X})[\AA]$ & $\mathrm{M}-\mathrm{Cl}$ & $\mathrm{M}-\mathrm{N}_{\mathrm{a}}$ & $\mathrm{M}-\mathrm{N}_{\mathrm{b}}$ \\
\hline$\left.[\mathrm{ThCl}((S)-\mathrm{PEBA}))_{3}\right](\mathbf{1})$ & $2.701(2)$ & $2.560(6)$ & $2.439(6)$ \\
{$\left[\mathrm{ThCl}\left(\mathrm{L}^{1}\right)_{3}\right]^{1}$} & $2.736(2)$ & $2.519(6)$ & $2.439(5)$ \\
{$\left[\mathrm{ThCl}\left(\mathrm{L}^{2}\right)_{3}\right]^{2}$} & $2.718(3)$ & $2.548(6)$ & $2.454(6)$ \\
\hline$\left[\mathrm{UCl}((S)-\mathrm{PEBA})_{3}\right](\mathbf{2})$ & $2.641(1)$ & $2.499(4)$ & $2.379(4)$ \\
{$\left[\mathrm{UCl}\left(\mathrm{L}^{3}\right)_{3}\right]^{3}$} & $2.678(1)$ & $2.457(3)$ & $2.395(3)$ \\
{$\left[\mathrm{UCl}\left(\mathrm{L}^{4}\right)_{3}\right]^{4}$} & $2.662(1)$ & $2.473(3)$ & $2.413(3)$ \\
{$\left[\mathrm{UCl}\left(\mathrm{L}^{5}\right)_{3}\right]^{5}$} & 2.659 & 2.448 & 2.414 \\
{$\left[\mathrm{UCl}\left(\mathrm{L}^{6}\right)_{3}\right]^{5}$} & 2.634 & 2.485 & 2.408 \\
\hline
\end{tabular}

$* \mathrm{~L}^{1}=N, N^{\prime}$-bis(isopropyl)methylamidinate; $\mathrm{L}^{2}=N, N^{\prime}$-bis(trimethylsilyl)-2-pyridylamidinate;

$\mathrm{L}^{3}=N, N^{\prime}$-bis(cyclohexyl)methylamidinate; $\mathrm{L}^{4}=N, N, N^{\prime}, N^{\prime \prime}$-tetra(isopropyl)guanidinate;

$\mathrm{L}^{5}=N, N^{\prime}$-bis(trimethylsilyl)benzamidinate; $\mathrm{L}^{6}=N, N^{\prime}$-bis(trimethylsilyl)-4-trifluormethylbenzamidinate; 
Table S7. Comparison of intramolecular distances of compound $\mathbf{4}$ with literature data of tetravalent cerium amidinate complexes. Bond lengths $\mathrm{M}-\mathrm{N} 1, \mathrm{M}-\mathrm{N} 3, \mathrm{M}-\mathrm{N} 5$ were averaged to $\mathrm{M}-\mathrm{N}_{\mathrm{a}}$ and distances $\mathrm{M}-$ N2, M-N4, M-N6 to M-N .

\begin{tabular}{llll}
\hline $\mathrm{d}(\mathrm{M}-\mathrm{X})[\AA]$ & $\mathrm{M}-\mathrm{Cl}$ & $\mathrm{M}-\mathrm{N}_{\mathrm{a}}$ & $\mathrm{M}-\mathrm{N}_{\mathrm{b}}$ \\
\hline$\left[\mathrm{CeCl}((S)-\mathrm{PEBA})_{3}\right](4)$ & $2.644(2)$ & $2.512(6)$ & $2.365(5)$ \\
{$\left[\mathrm{CeCl}\left(\mathrm{L}^{7}\right)_{3}\right]^{6}$} & $2.655(1)$ & $2.470(2)$ & $2.393(2)$ \\
{$\left[\mathrm{Ce}\left(\mathrm{L}^{8}\right)_{3}\right]\left[\mathrm{BAr}{ }_{4}\right]^{7}$} & & $2.526(4)$ & $2.527(4)$ \\
{$\left[\mathrm{CeCl}\left(\mathrm{L}^{8}\right)\left(\mathrm{N}\left(\mathrm{SiMe}_{3}\right)_{2}\right)_{2}\right]^{8}$} & & $2.439(2)$ & $2.405(2)$ \\
{$\left[\mathrm{CeCl}\left(\mathrm{L}^{9}\right)\left(\mathrm{N}\left(\mathrm{SiMe}_{3}\right)_{2}\right)_{2}\right]^{8}$} & & $2.434(2)$ & $2.402(2)$ \\
{$\left[\mathrm{Ce}\left(\mathrm{L}^{10}\right)_{4}\right]^{9}$} & & $2.464(2)$ & $2.430(2)$ \\
\hline
\end{tabular}

$* \mathrm{~L}^{7}=N, N^{\prime}$-bis(trimethylsilyl)-4-methoxy-benzamidinate;

$\mathrm{L}^{8}=N, N^{\prime}$-di(isopropyl)- $N^{\prime \prime}, N^{\prime \prime}$-bis(trimethylsilyl)guanidinate;

$\mathrm{Ar}^{\mathrm{F}}=3,5$-bis(trifluormethyl)phenyl;

$\mathrm{L}^{9}=N, N^{\prime}$-di(cyclohexyl)- $N^{\prime \prime}, N^{\prime \prime}$-bis(trimethylsilyl)guanidinate;

$\mathrm{L}^{10}=N, N^{\prime}$-bis(p-tolyl)formamidinate

Table S8. Comparison of intramolecular distance $\mathrm{M}-\mathrm{N}_{\mathrm{az}}$ and angle $\mathrm{M}-\mathrm{N}-\mathrm{N}$ of azido compounds 6 and 7 with literature data of terminal tetravalent actinide monoazido compounds.

\begin{tabular}{lll}
\hline & $\mathrm{M}-\mathrm{N}_{\mathrm{az}}[\AA]$ & $\angle \mathrm{M}-\mathrm{N}-\mathrm{N}\left[^{\circ}\right]$ \\
\hline$\left[\mathrm{ThN}_{3}((\mathrm{~S})-\mathrm{PEBA})_{3}\right](6)$ & $2.342(16)$ & $160.1(15)$ \\
{$\left[\mathrm{ThN}_{3} \mathrm{Cp}^{*}{ }_{2}\left(\mathrm{~N}(\mathrm{SiMe})_{2}\right)\right]^{10}$} & $2.301(8)$ & $166.1(9)$ \\
$\left.\mathrm{ThN}_{3} \mathrm{Cp}^{\prime \prime \prime}{ }_{2}(\mathrm{NPMes})\right]^{11}$ & $2.294(7)$ & $166.2(7)$ \\
\hline$\left[\mathrm{UN}_{3}((S)-\mathrm{PEBA})_{3}\right](7)$ & $2.306(9)$ & $172.9(8)$ \\
{$\left[\mathrm{UN}_{3}\left(\mathrm{~L}^{4}\right)_{3}\right]^{4}$} & $2.293(6)$ & $155.7(5)$ \\
{$\left[\mathrm{UN}_{3} \mathrm{~L}^{11}\right]^{12}$} & $2.326(2)$ & $167.8(2)$ \\
{$\left[\mathrm{UN}_{3} \mathrm{~L}^{12}(\mathrm{dme})\right]^{13}$} & $2.372(3)$ & $175.6(3)$ \\
{$\left[\mathrm{UN}_{3} \mathrm{~L}^{13}\right]^{14}$} & $2.422(4)$ & $151.8(3)$ \\
{$\left[\mathrm{UN}_{3}\left(\mathrm{~L}^{14}\right)\right]^{15}$} & $2.260(7)$ & $168.1(5)$ \\
{$\left[\mathrm{UN}_{3} \mathrm{Cp}^{*}{ }_{2}\left(\mathrm{~N}(\mathrm{TMS})_{2}\right)\right]^{16}$} & $2.305(3)$ & $176.0(3)$ \\
{$\left[\mathrm{UN}_{3} \mathrm{Cp}^{*}{ }_{2}(\mathrm{O}-2,6-i \mathrm{Pr}-\mathrm{Ph})\right]^{16}$} & $2.30(2)$ & $164(2)$ \\
{$\left[\mathrm{UN}_{3} \mathrm{Cp}_{2}{ }_{2} \mathrm{~L}^{15}\right]^{17}$} & $2.26(2)$ & $163.5(17)$ \\
\hline
\end{tabular}

$* \mathrm{Cp}^{*}=\eta^{5}-\mathrm{C}_{5} \mathrm{Me}_{5}$;

$\mathrm{Cp}^{\prime \prime \prime}=\eta^{5}-1,2,4-\left(\mathrm{Me}_{3} \mathrm{C}\right)_{3} \mathrm{C}_{5} \mathrm{H}_{2}$;

Mes* = 2,4,6-( $\left.\mathrm{Me}_{3} \mathrm{C}\right)_{3} \mathrm{C}_{6} \mathrm{H}_{2}$;

$\mathrm{L}^{4}=N, N, N^{\prime}, N^{\prime \prime}$-tetra(isopropyl)guanidinate;

$\mathrm{L}^{11}=$ 1,4,7-tris(3-adamantyl-5-tert-butyl-2-hydroxybenzyl)-1,4,7-triazacyclononane;

$\mathrm{L}^{12}=$ Tris(2-hydroxy-3-adamantyl-5-methylbenzyl)amine;

$\mathrm{L}^{13}=N, N^{\prime}, N^{\prime \prime}$-tris-((tris-phenyl)silyl)amidotriethylamine;

$\mathrm{L}^{14}=N, N^{\prime}, N^{\prime \prime}$-tris-((tris-isopropyl)silyl)-amidotriethylamine;

$\mathrm{L}^{15}=N, N^{\prime}-1,3,4,6,7,8$-hexahydro-2H-pyrimido(1,2-a)pyrimidinate 
Table S9. Averaged bond lengths (experimental from SC-XRD and calculated from geometry optimization) in reported tris(amidinate) $\left[\mathrm{MX}(\mathrm{L})_{3}\right]\left(\mathrm{M}=\mathrm{Th}, \mathrm{Pa}, \mathrm{U}, \mathrm{Np}, \mathrm{Ce}, \mathrm{Pu} ; \mathrm{X}=\mathrm{Cl}, \mathrm{N}_{3} ; \mathrm{L}=(S)\right.$-PEBA) compounds. Values in brackets correspond to standard deviation of average for $\mathrm{N}_{\mathrm{a}}\left(\mathrm{M}-\mathrm{N} 1, \mathrm{~N} 3\right.$ and N5) and $\mathrm{N}_{\mathrm{b}}(\mathrm{M}-$ $\mathrm{N} 2$, N4 and N6) but indicate crystallographic error for M-X.

\begin{tabular}{lccccccc}
\hline & \multirow{2}{*}{ IR $[\AA]$ SC-XRD } & \multicolumn{3}{c}{ Optimization } \\
\cline { 3 - 8 } & & $\mathrm{M}-\mathrm{X}$ & $\mathrm{M}-\mathrm{N}_{\mathrm{a}}$ & $\mathrm{M}-\mathrm{N}_{\mathrm{b}}$ & $\mathrm{M}-\mathrm{X}$ & $\mathrm{M}-\mathrm{N}_{\mathrm{a}}$ & $\mathrm{M}-\mathrm{N}_{\mathrm{b}}$ \\
\hline$\left[\mathrm{ThCl}(\mathrm{L})_{3}\right](\mathbf{1})$ & 1.00 & $2.701(1)$ & $2.560(12)$ & $2.439(13)$ & 2.726 & $2.547(1)$ & $2.449(1)$ \\
{$\left[\mathrm{PaCl}(\mathrm{L})_{3}\right]$} & 0.96 & - & - & - & 2.696 & $2.496(2)$ & $2.416(1)$ \\
{$\left[\mathrm{UCl}(\mathrm{L})_{3}\right](\mathbf{2})$} & 0.95 & $2.641(1)$ & $2.499(4)$ & $2.379(19)$ & 2.673 & $2.488(17)$ & $2.392(11)$ \\
{$\left[\mathrm{NpCl}(\mathrm{L})_{3}\right](\mathbf{3})^{18}$} & 0.93 & $2.630(1)$ & $2.497(4)$ & $2.368(15)$ & 2.664 & $2.479(5)$ & $2.390(6)$ \\
{$\left[\mathrm{CeCl}(\mathrm{L})_{3}\right](\mathbf{4})$} & 0.92 & $2.644(2)$ & $2.512(6)$ & $2.365(20)$ & 2.705 & $2.513(8)$ & $2.420(1)$ \\
{$\left[\mathrm{PuCl}(\mathrm{L})_{3}\right]$} & 0.91 & - & - & - & 2.705 & $2.428(3)$ & $2.426(2)$ \\
\hline$\left[\mathrm{ThN}_{3}(\mathrm{~L})_{3}\right](\mathbf{6})$ & 1.00 & $2.342(16)$ & $2.560(4)$ & $2.430(20)$ & 2.376 & $2.540(23)$ & $2.459(8)$ \\
{$\left[\mathrm{PaN}_{3}(\mathrm{~L})_{3}\right]$} & 0.96 & - & - & - & 2.340 & $2.503(7)$ & $2.414(1)$ \\
{$\left[\mathrm{UN}_{3}(\mathrm{~L})_{3}\right](\mathbf{7})$} & 0.95 & $2.293(6)$ & $2.494(12)$ & $2.389(10)$ & 2.320 & $2.490(30)$ & $2.396(12)$ \\
{$\left[\mathrm{NpN}_{3}(\mathrm{~L})_{3}\right](\mathbf{8})^{18}$} & 0.93 & $2.230(30)$ & $2.490(36)$ & $2.380(26)$ & 2.304 & $2.492(6)$ & $2.381(7)$ \\
{$\left[\mathrm{CeN}_{3}(\mathrm{~L})_{3}\right]$} & 0.92 & - & - & - & 2.355 & $2.516(1)$ & $2.414(1)$ \\
{$\left[\mathrm{PuN}_{3}(\mathrm{~L})_{3}\right]$} & 0.91 & - & - & - & 2.335 & $2.436(4)$ & $2.420(2)$ \\
\hline
\end{tabular}


Table S10. Crystallographic data of 1-4.

\begin{tabular}{|c|c|c|c|c|}
\hline & 1 & 2 & $3^{18}$ & 4 \\
\hline Empirical formula & {$\left[\mathrm{C}_{69} \mathrm{H}_{69} \mathrm{ClN}_{6} \mathrm{Th}\right] \mathrm{C}_{7} \mathrm{H}_{8}$} & {$\left[\mathrm{C}_{69} \mathrm{H}_{69} \mathrm{ClN}_{6} \mathrm{U}\right] \mathrm{C}_{7} \mathrm{H}_{8}$} & {$\left[\mathrm{C}_{69} \mathrm{H}_{69} \mathrm{ClN}_{6} \mathrm{~Np}\right] \mathrm{C}_{7} \mathrm{H}_{8}$} & {$\left[\mathrm{C}_{69} \mathrm{H}_{69} \mathrm{ClN}_{6} \mathrm{Ce}\right] \mathrm{C}_{7} \mathrm{H}_{8}$} \\
\hline CCDC number & 1884772 & 1884773 & 1884774 & 1884776 \\
\hline M (g/mol) & 1341.93 & 1347.92 & 1041.42 & 1250.01 \\
\hline Crystal system & Orthorhombic & Orthorhombic & Orthorhombic & Orthorhombic \\
\hline Space group & $P 2{ }_{1} 2_{1} 2_{1}$ & $P 2_{1} 2_{1} 2_{1}$ & $P 2_{1} 2_{1} 2_{1}$ & $P 2{ }_{1} 2_{1} 2_{1}$ \\
\hline$a(\AA)$ & $11.352(2)$ & $11.239(1)$ & $11.274(2)$ & $11.271(1)$ \\
\hline$b(\AA)$ & $15.435(2)$ & $15.366(2)$ & $15.356(2)$ & $15.390(2)$ \\
\hline$c(\AA)$ & $36.744(6)$ & $36.704(3)$ & $36.654(5)$ & $36.744(3)$ \\
\hline$V\left(\AA^{3}\right)$ & $6438.2(17)$ & $6338.5(10)$ & $6345.8(15)$ & $6373.6(10)$ \\
\hline$T(\mathbf{K})$ & 100 & 100 & 100 & 100 \\
\hline$Z$ & 4 & 4 & 4 & 4 \\
\hline$\rho_{\text {calcd }}\left(\mathrm{Mg} / \mathrm{m}^{3}\right)$ & 1.385 & 1.413 & 1.410 & 1.303 \\
\hline $\operatorname{abs} \operatorname{coeff}\left(\mathrm{mm}^{-1}\right)$ & 2.405 & 2.652 & 1.728 & 0.804 \\
\hline$\Theta_{\max }\left({ }^{\circ}\right)$ & 25.05 & 26.02 & 25.04 & 25.03 \\
\hline$R[I>2 \sigma(I)]$ & 0.036 & 0.029 & 0.031 & 0.048 \\
\hline wR2(int) & 0.068 & 0.052 & 0.101 & 0.081 \\
\hline$w$ scheme $d, e$ & $0.0207,0$ & $0.0150,6.0873$ & $0.0343,8.9548$ & $0.0142,13.9475$ \\
\hline Data/Param & $11382 / 717$ & $12459 / 759$ & $11206 / 752$ & $11238 / 746$ \\
\hline res. Dens $\left(\mathrm{e}^{-3}\right)$ & $1.12,-1.75$ & $0.72,-0.92$ & $1.39,-2.01$ & $0.77,-0.61$ \\
\hline $\mathbf{R}_{\text {int }}$ & 0.069 & 0.083 & 0.043 & 0.1211 \\
\hline GooF & 1.029 & 1.022 & 1.137 & 1.037 \\
\hline Flack x & $0.032(4)$ & $0.016(2)$ & $0.014(4)$ & $0.022(6)$ \\
\hline
\end{tabular}


Table S11. Crystallographic data of 5-8.

\begin{tabular}{|c|c|c|c|c|}
\hline & 5 & 6 & 7 & $\mathbf{8}^{18}$ \\
\hline Empirical formula & {$\left[\mathrm{C}_{69} \mathrm{H}_{69} \mathrm{~N}_{6} \mathrm{Ce}\right]$} & {$\left[\mathrm{C}_{69} \mathrm{H}_{69} \mathrm{~N}_{9} \mathrm{Th}\right] 0.5 \mathrm{C}_{7} \mathrm{H}_{8}$} & {$\left[\mathrm{C}_{69} \mathrm{H}_{69} \mathrm{~N}_{9} \mathrm{U}\right] \mathrm{C}_{7} \mathrm{H}_{8}$} & {$\left[\mathrm{C}_{69} \mathrm{H}_{69} \mathrm{~N}_{9} \mathrm{~Np}\right] \mathrm{C}_{7} \mathrm{H}_{8}$} \\
\hline CCDC number & 1884775 & 1936872 & 1936873 & 1936874 \\
\hline M (g/mol) & 936.23 & 1302.43 & 1354.50 & 1353.46 \\
\hline Crystal system & Cubic & Orthorhombic & Orthorhombic & Orthorhombic \\
\hline Space group & $P 2_{1} 3$ & $P 2_{1} 2_{1} 2_{1}$ & $P 2_{1} 2_{1} 2_{1}$ & $P 2_{1} 2_{1} 2_{1}$ \\
\hline$a(\AA)$ & $18.5568(5)$ & $11.443(3)$ & $11.406(2)$ & $11.454(3)$ \\
\hline$b(\AA)$ & $18.5568(5)$ & $15.396(3)$ & $15.961(3)$ & $15.975(4)$ \\
\hline$c(\AA)$ & $18.5568(5)$ & $37.274(8)$ & $36.196(6)$ & $36.264(9)$ \\
\hline$V\left(\AA^{3}\right)$ & $6390.1(5)$ & $6567(3)$ & $6590(2)$ & $6636(3)$ \\
\hline$T(\mathbf{K})$ & 100 & 100 & 100 & 100 \\
\hline$Z$ & 4 & 4 & 4 & 4 \\
\hline$\rho_{\text {calcd }}\left(\mathrm{Mg} / \mathrm{m}^{3}\right)$ & 1.167 & 1.317 & 1.365 & 1.355 \\
\hline $\operatorname{abs} \operatorname{coeff}\left(\mathrm{mm}^{-1}\right)$ & 0.755 & 2.318 & 2.513 & 1.615 \\
\hline$\Theta_{\max }\left({ }^{\circ}\right)$ & 28.28 & 25.03 & 26.02 & 19.780 \\
\hline$R[I>2 \sigma(I)]$ & 0.038 & 0.073 & 0.036 & 0.0904 \\
\hline wR2(int) & 0.110 & 0.205 & 0.086 & 0.2448 \\
\hline$w$ scheme $d, e$ & $0.0754,2.0138$ & $0,181.0673$ & $0.0068 / 21.0648$ & $0,365.3019$ \\
\hline Data/Param & $5291 / 231$ & $11565 / 692$ & $12879 / 782$ & $5548 / 631$ \\
\hline res. Dens $\left(\mathbf{e}^{-3}\right)$ & $1.54,-0.47$ & $4.267 /-7.528$ & $1.68 /-2.80$ & $3.145 /-1.684$ \\
\hline $\mathbf{R}_{\text {int }}$ & 0.029 & 0.0451 & 0.047 & 0.0914 \\
\hline GooF & 1.099 & 1.372 & 1.248 & 1.147 \\
\hline Flack $x$ & $0.000(5)$ & $0.11(2)$ & $0.072(2)$ & $0.033(17)$ \\
\hline
\end{tabular}




\section{NMR spectra}

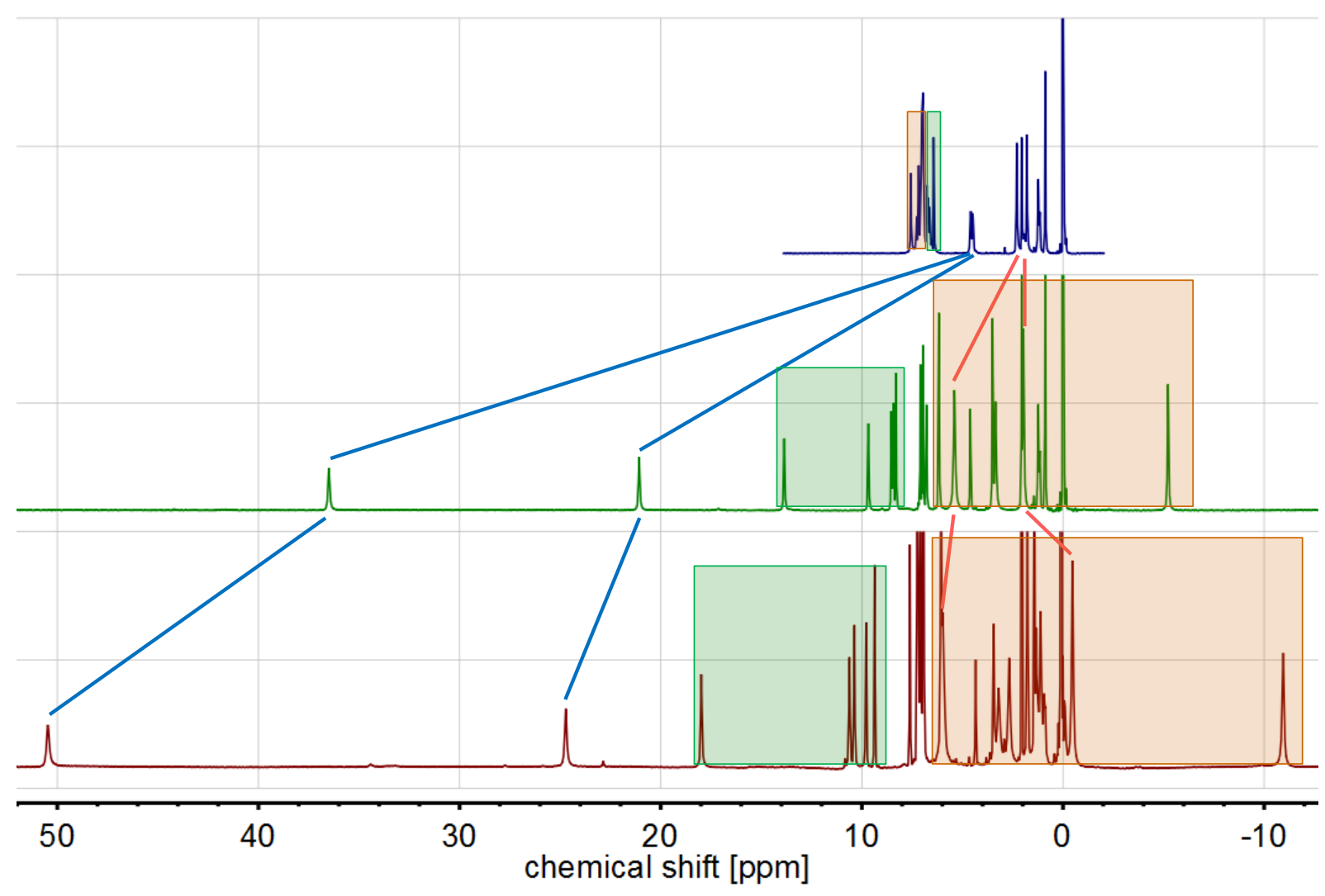

Figure S11. ${ }^{1} \mathrm{H}-\mathrm{NMR}$ spectra of $\left[\mathrm{ThCl}((S)-\mathrm{PEBA})_{3}\right](\mathbf{1}$, top $),\left[\mathrm{UCl}((S)-\mathrm{PEBA})_{3}\right](\mathbf{2}$, middle $),\left[\mathrm{NpCl}((S)-\mathrm{PEBA})_{3}\right]$ $\left(3\right.$, bottom) measured at $243 \mathrm{~K}$ in toluene- $\mathrm{d}_{8}$. Blue lines indicate signal shift for $\mathrm{NCH}$, whereas red lines correspond to methyl groups. Green and brown boxes are dedicated to signals of central (former) and terminal phenyl groups (latter). 




Figure S12. ${ }^{1} \mathrm{H}-\mathrm{NMR}$ spectrum of $\left[\mathrm{ThCl}((S)-\mathrm{PEBA})_{3}\right](\mathbf{1})$ in toluene- $\mathrm{d}_{8}$ at $243 \mathrm{~K}$ with signal assignments. Dagger $(\dagger)$ indicates impurity of $n$-pentane from washing procedure.

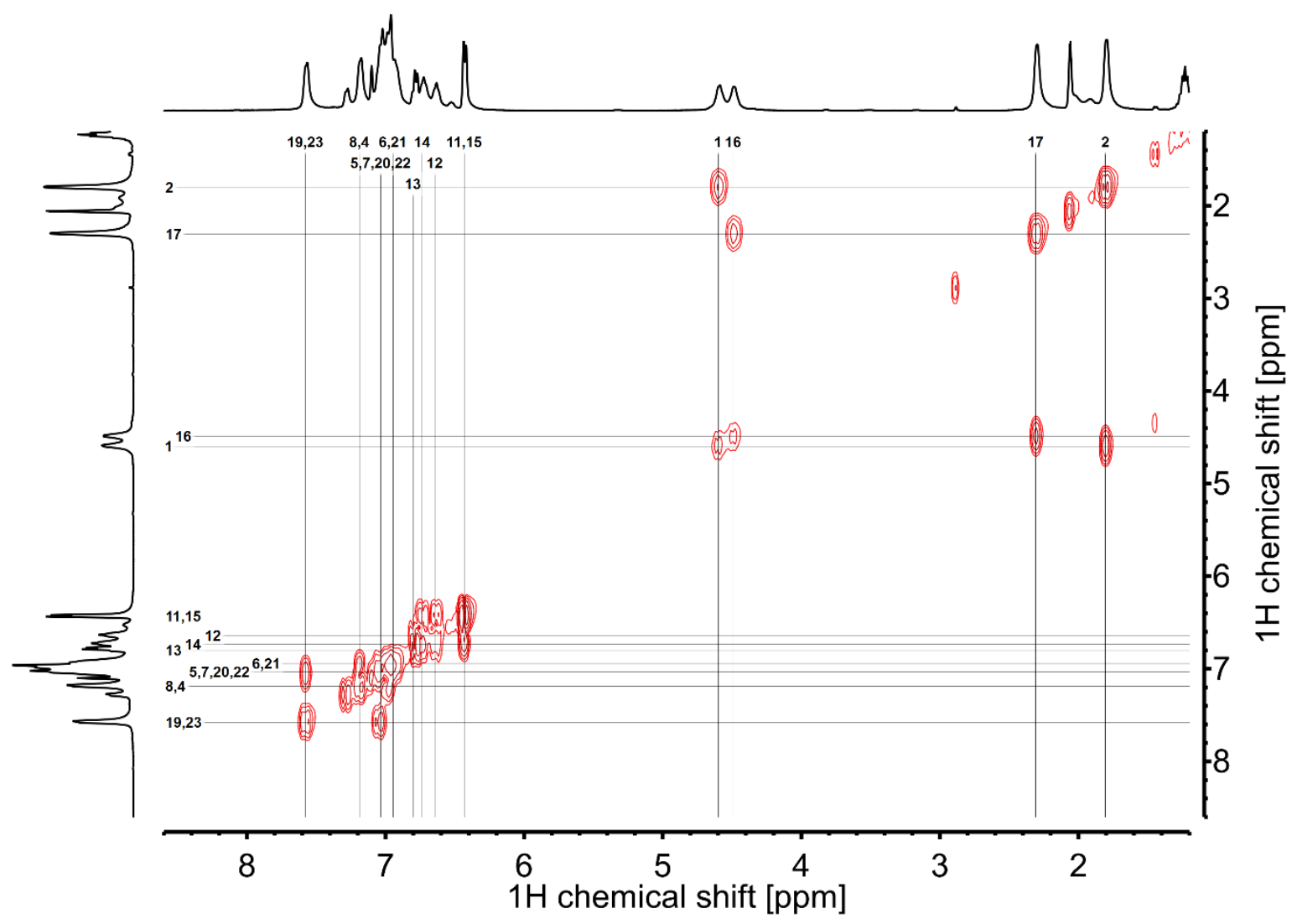

Figure S13. ${ }^{1} \mathrm{H}-{ }^{1} \mathrm{H}-\mathrm{COSY}$ spectrum of $\left[\mathrm{ThCl}((S)-\mathrm{PEBA})_{3}\right](\mathbf{1})$ in toluene- $\mathrm{d}_{8}$ at $243 \mathrm{~K}$ with signal assignments. 


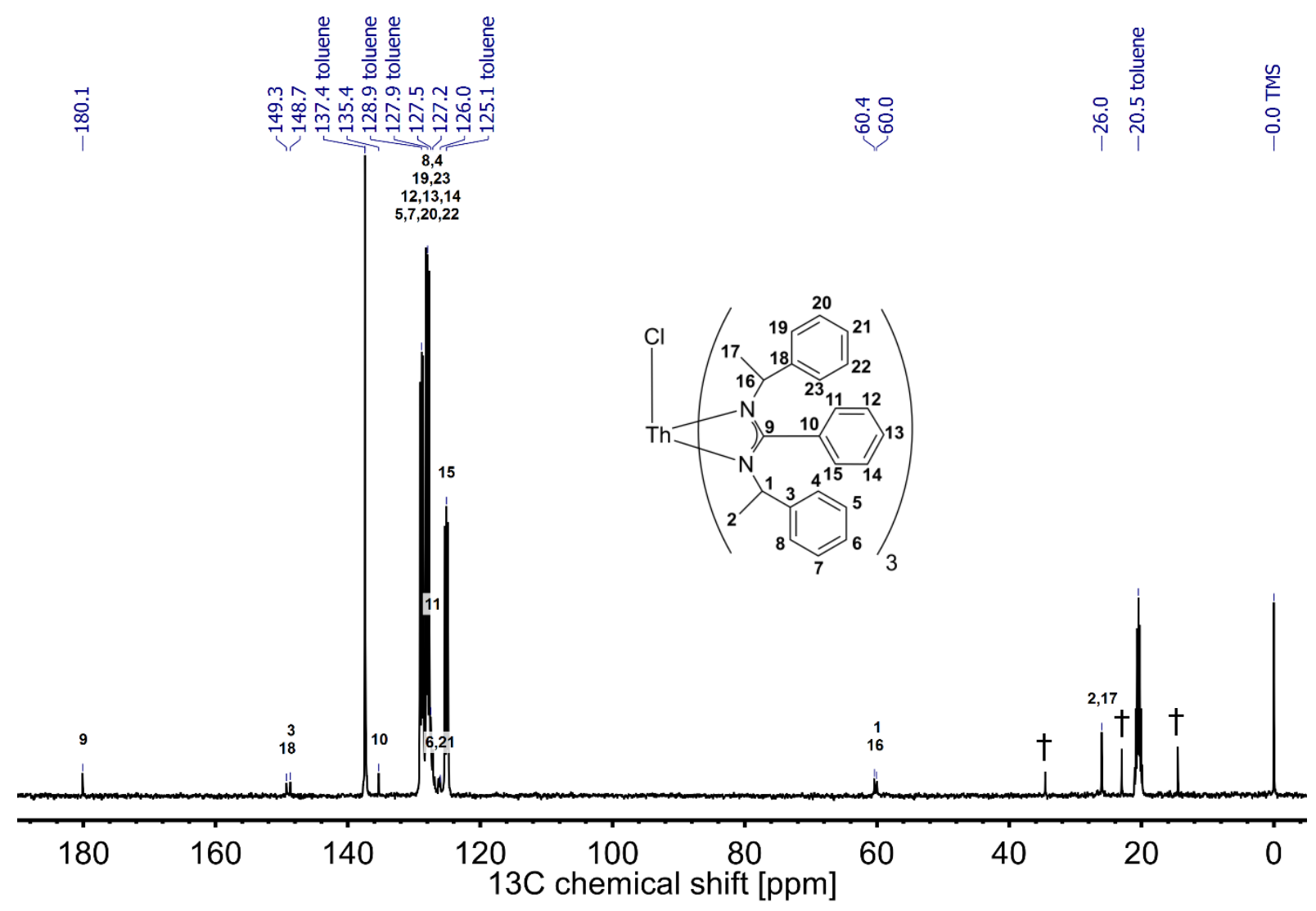

Figure S14. ${ }^{13} \mathrm{C}-\mathrm{NMR}$ spectrum of $\left[\mathrm{ThCl}((S)-\mathrm{PEBA})_{3}\right](\mathbf{1})$ in toluene- $\mathrm{d}_{8}$ at $243 \mathrm{~K}$. Dagger $(\dagger)$ indicates impurity of $n$-pentane from washing procedure.

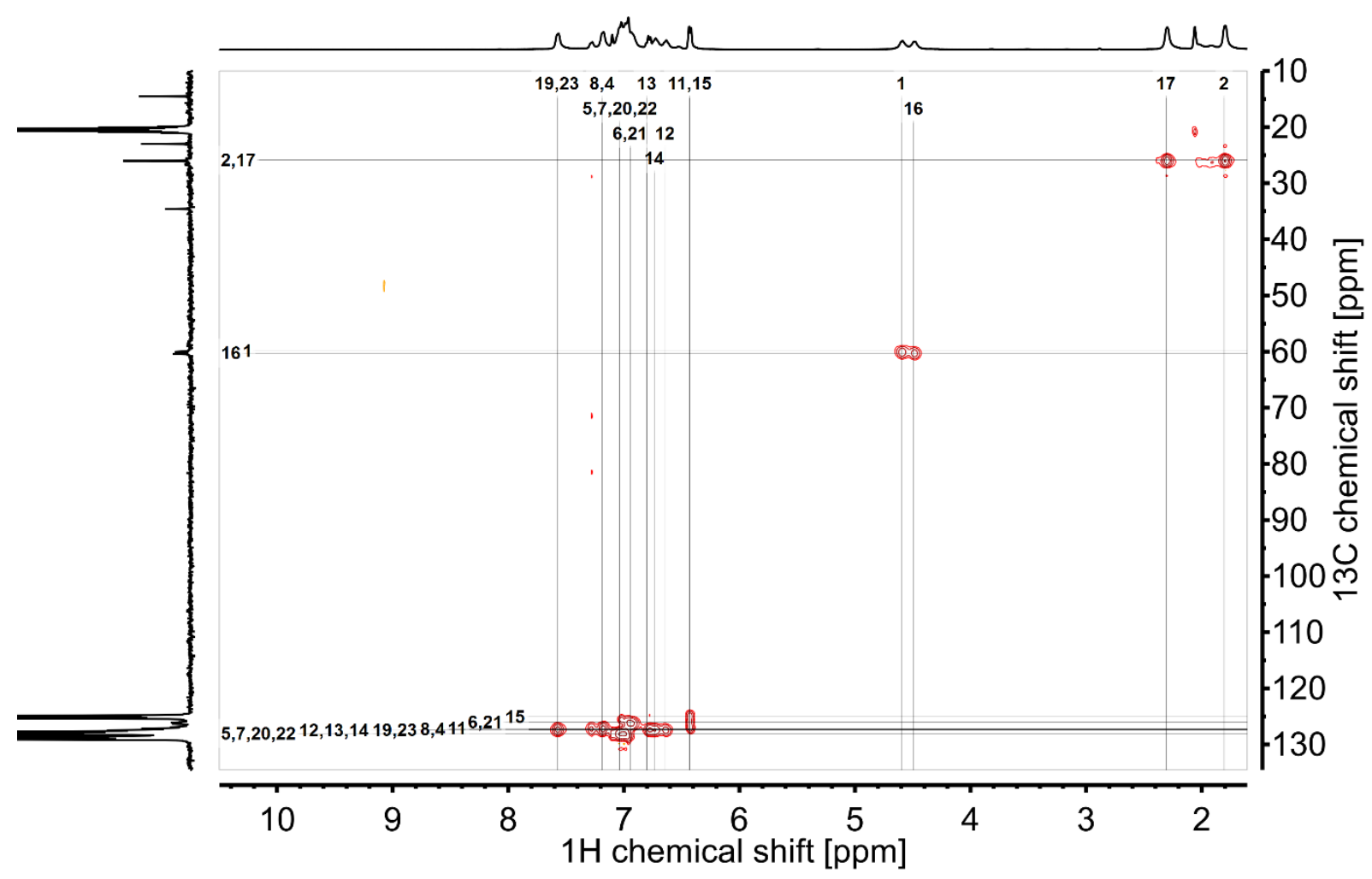

Figure S15. ${ }^{1} \mathrm{H}^{13} \mathrm{C}-\mathrm{HSQC}$ spectrum of $\left[\mathrm{ThCl}((S)-\mathrm{PEBA})_{3}\right](\mathbf{1})$ in toluene-d $\mathrm{d}_{8}$ at $243 \mathrm{~K}$ with signal assignments. 


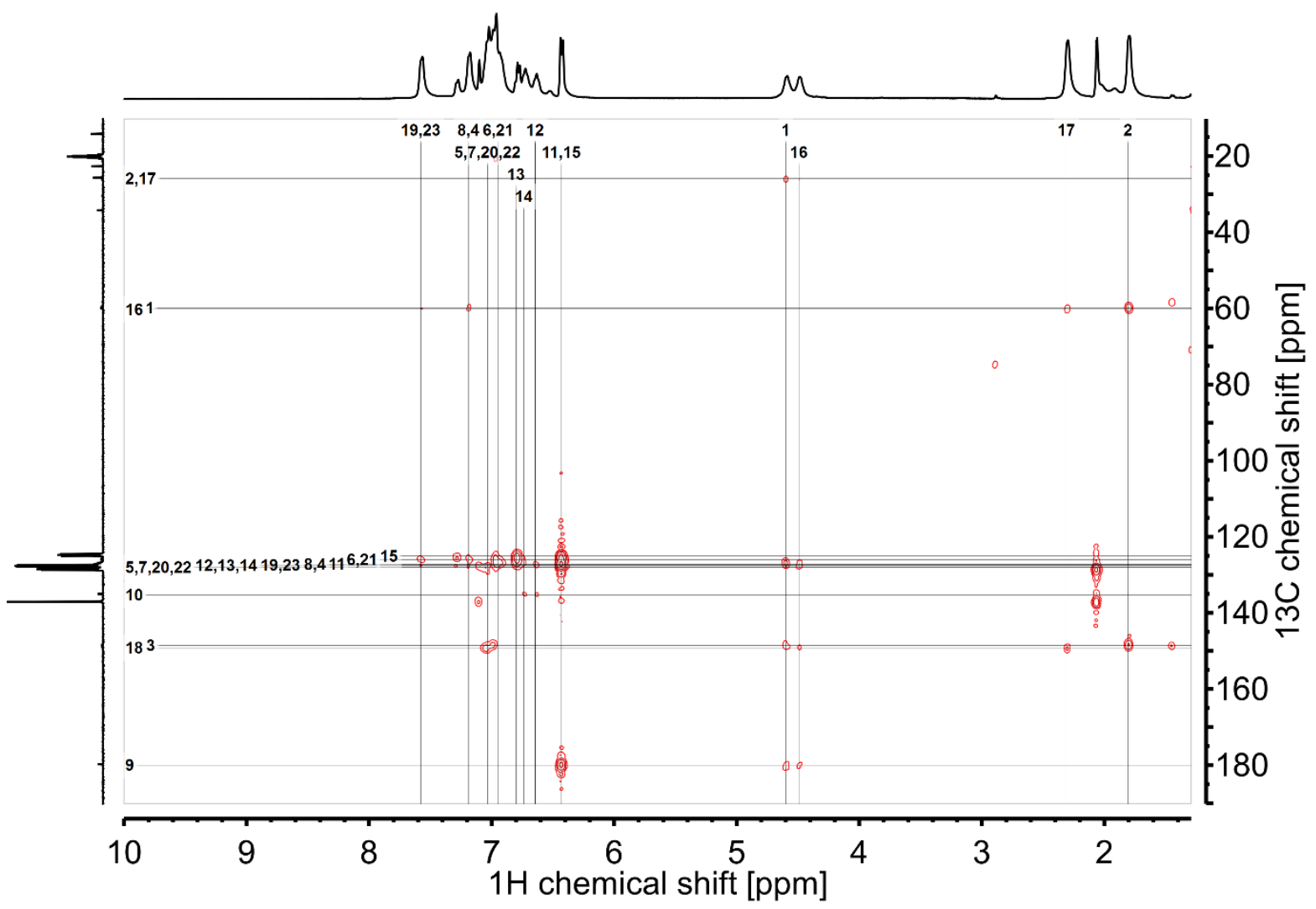

Figure S16. ${ }^{1} \mathrm{H}-{ }^{13} \mathrm{C}-\mathrm{HMBC}$ spectrum of $\left[\mathrm{ThCl}((S)-\mathrm{PEBA})_{3}\right](\mathbf{1})$ in toluene- $\mathrm{d}_{8}$ at $243 \mathrm{~K}$ with signal assignments. 

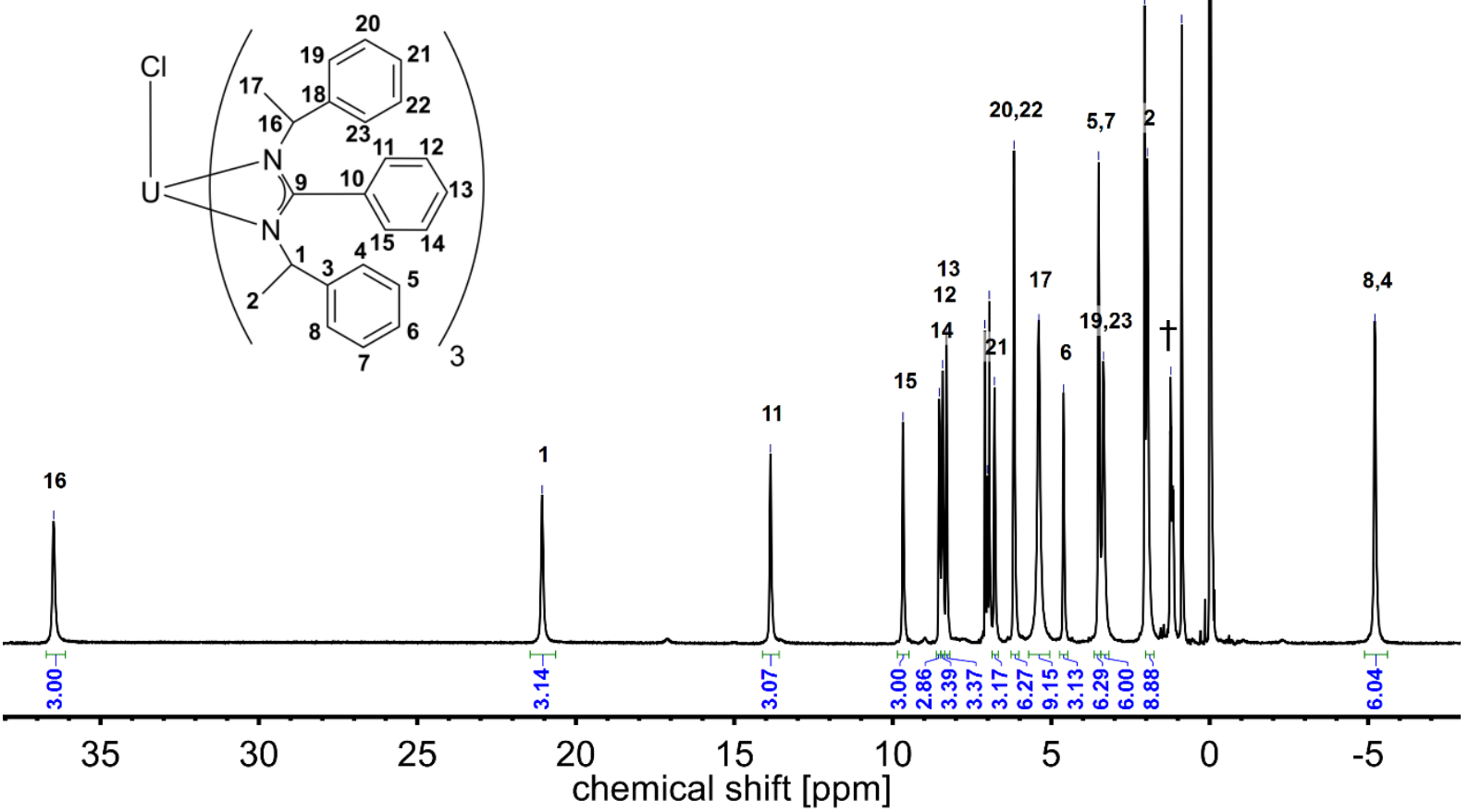

Figure S17. ${ }^{1} \mathrm{H}-\mathrm{NMR}$ spectrum of $\left[\mathrm{UCl}((S)-\mathrm{PEBA})_{3}\right](2)$ in toluene- $\mathrm{d}_{8}$ at $243 \mathrm{~K}$ with signal assignments. Dagger $(\dagger)$ indicates impurity of $n$-pentane from washing procedure.

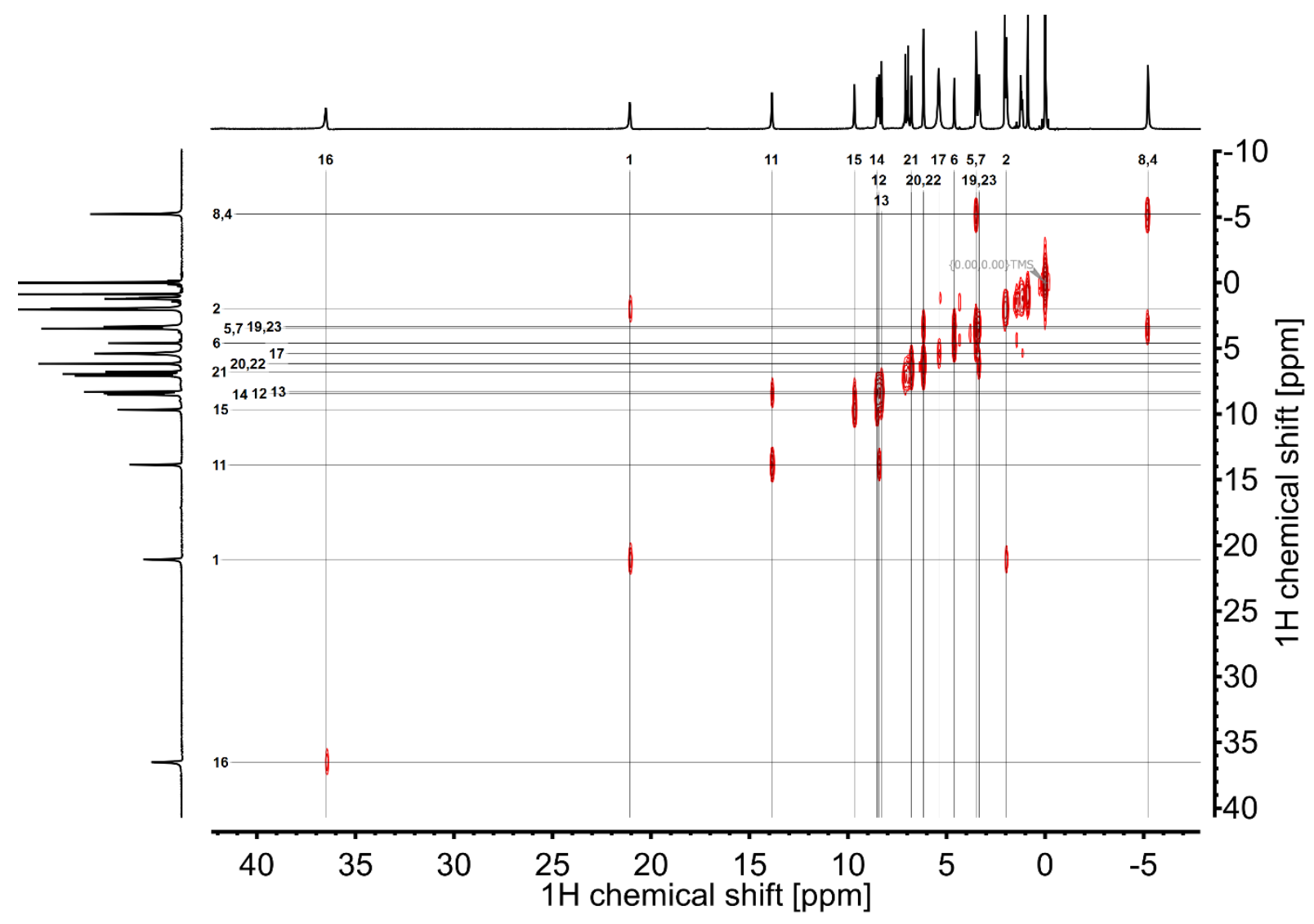

Figure S18. ${ }^{1} \mathrm{H}-{ }^{1} \mathrm{H}-\mathrm{COSY}$ spectrum of $\left[\mathrm{UCl}((S)-\mathrm{PEBA})_{3}\right](2)$ in toluene- $\mathrm{d}_{8}$ at $243 \mathrm{~K}$ with signal assignments. 


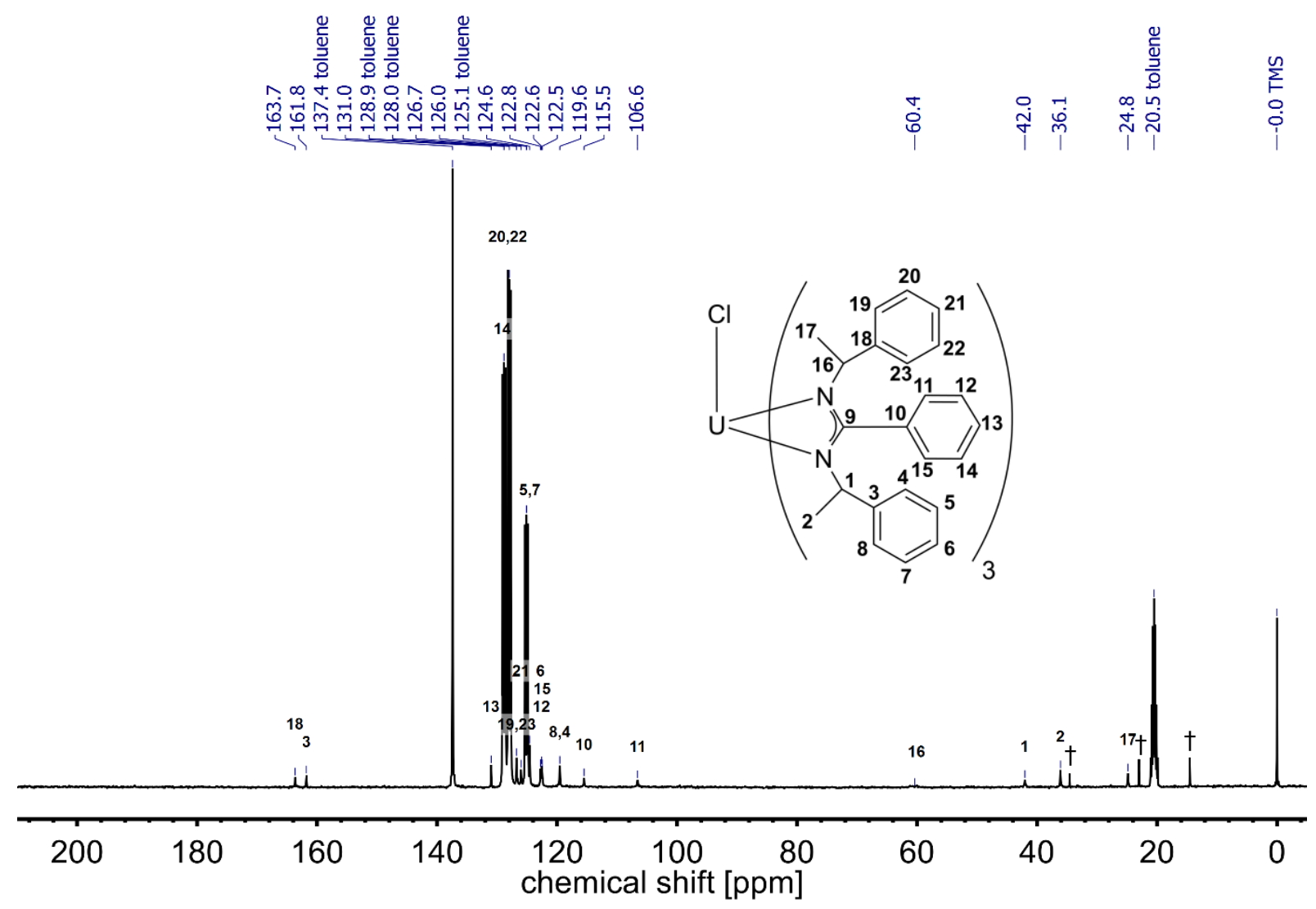

Figure S19. ${ }^{13} \mathrm{C}-\mathrm{NMR}$ spectrum of $\left[\mathrm{UCl}((S)-\mathrm{PEBA})_{3}\right](2)$ in toluene- $\mathrm{d}_{8}$ at $243 \mathrm{~K}$ with signal assignments. Dagger $(\dagger)$ indicates impurity of $n$-pentane from washing procedure.

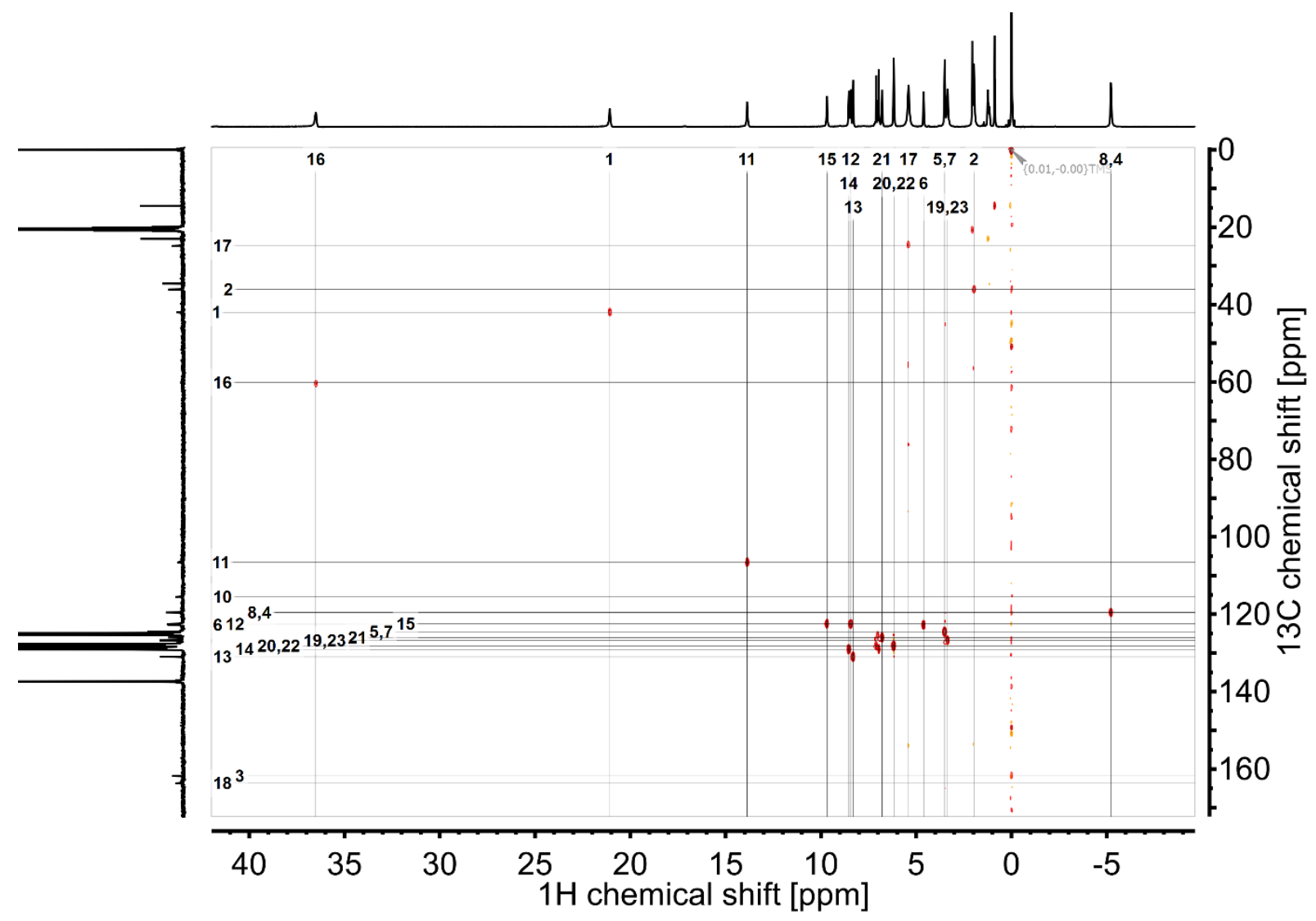

Figure S20. ${ }^{1} \mathrm{H}_{-}{ }^{13} \mathrm{C}-\mathrm{HSQC}$ spectrum of $\left[\mathrm{UCl}((S)-\mathrm{PEBA})_{3}\right](\mathbf{2})$ in toluene-d $\mathrm{d}_{8}$ at $243 \mathrm{~K}$ with signal assignments. 


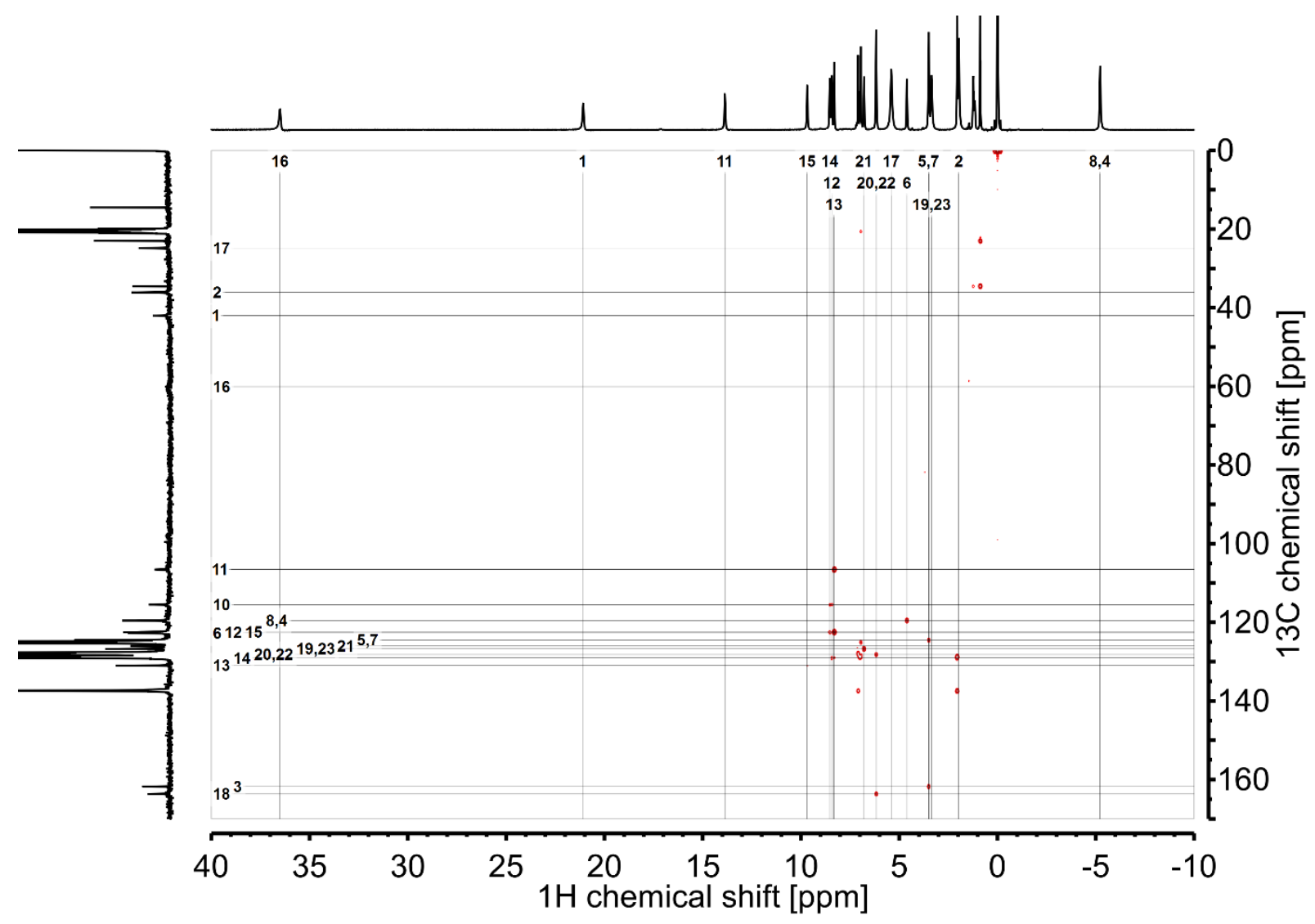

Figure S21. ${ }^{1} \mathrm{H}-{ }^{13} \mathrm{C}-\mathrm{HMBC}$ spectrum of $\left[\mathrm{ThCl}((S)-\mathrm{PEBA})_{3}\right](\mathbf{1})$ in toluene- $\mathrm{d}_{8}$ at $243 \mathrm{~K}$ with signal assignments. 


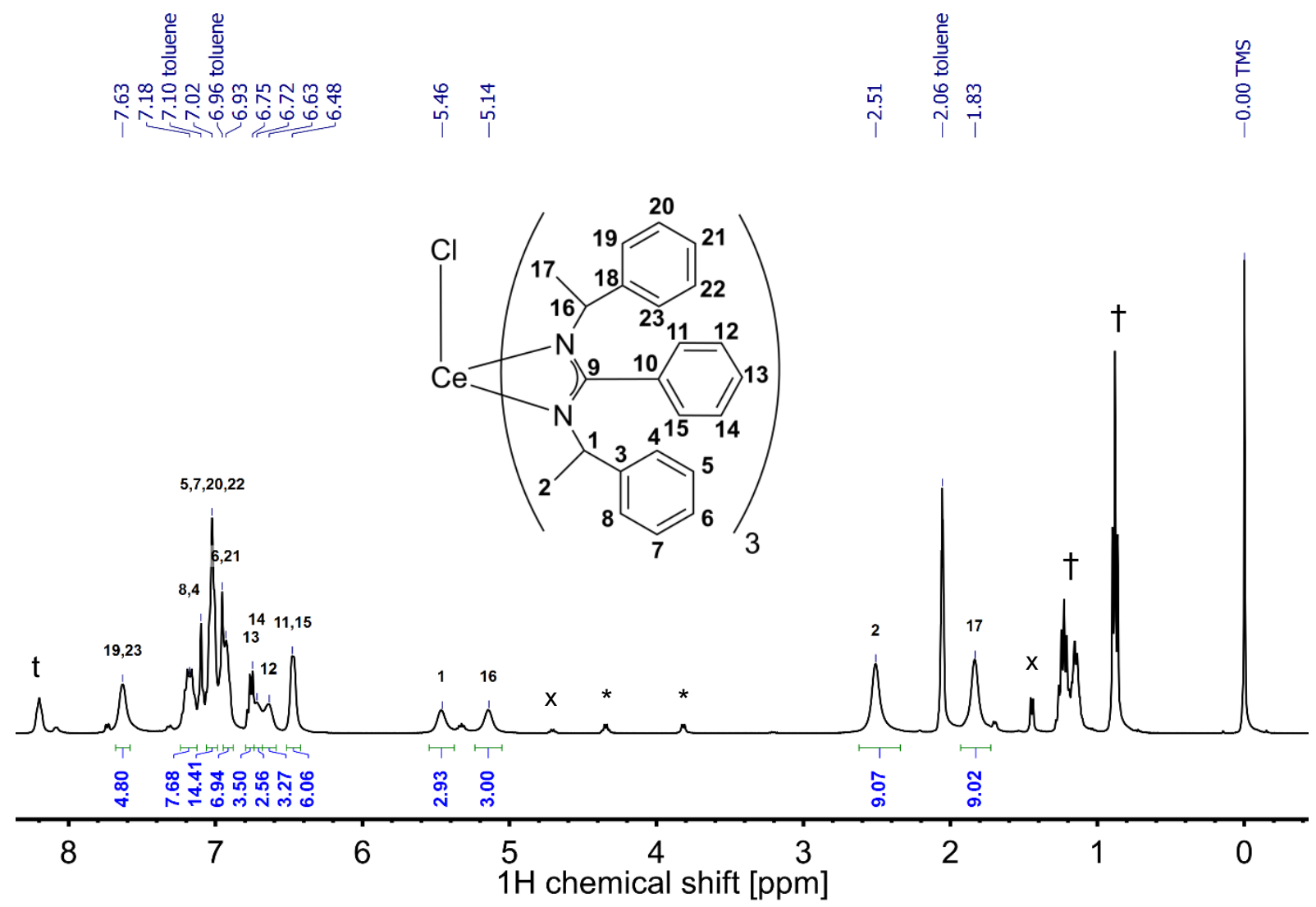

Figure S22. ${ }^{1} \mathrm{H}-\mathrm{NMR}$ spectrum of dark blue product after oxidation of $\left[\mathrm{Ce}((S)-\mathrm{PEBA})_{3}\right](5)$ with $\mathrm{PhICl}_{2}$ evidencing the presence of $\left[\mathrm{CeCl}((S)-\mathrm{PEBA})_{3}\right](4)$ in toluene- $\mathrm{d}_{8}$ at $243 \mathrm{~K}$ with assignments. Impurities are marked: $\uparrow-n$-pentane; $*-(S)$-HPEBA; $\mathrm{x}-(S)$-LiPEBA; $\mathrm{t}-\left[\mathrm{Ce}((\mathrm{S}) \text {-PEBA })_{3}\right](\mathbf{5})$.

The ${ }^{1} \mathrm{H}-\mathrm{NMR}$ spectrum of the blue product after the oxidation of $\left[\mathrm{Ce}((S) \text {-PEBA })_{3}\right](5)$ evidenced the formation of the tetravalent $\left[\mathrm{CeCl}((S) \text {-PEBA })_{3}\right](4)$ complex which has also been proven by analysis of the SC-XRD structure. However, the spectrum of $\mathbf{4}$ contains several impurities which are mainly due to the extreme sensitivity of $\mathbf{4}$ towards any kind of protic impurity or redoxactive compound. Thus, it cannot be determined whether the presence of the trivalent cerium complex $\mathbf{5}$ is due to the already happened reduction of $\mathbf{4}$ or due to the incomplete oxidation of $\mathbf{5}$ to $\mathbf{4}$. Further work-up of $\mathbf{4}$ was hampered by the similar solubility of 4, 5, $(S)$-HPEBA, and $(S)$-LiPEBA in aprotic solvents. 


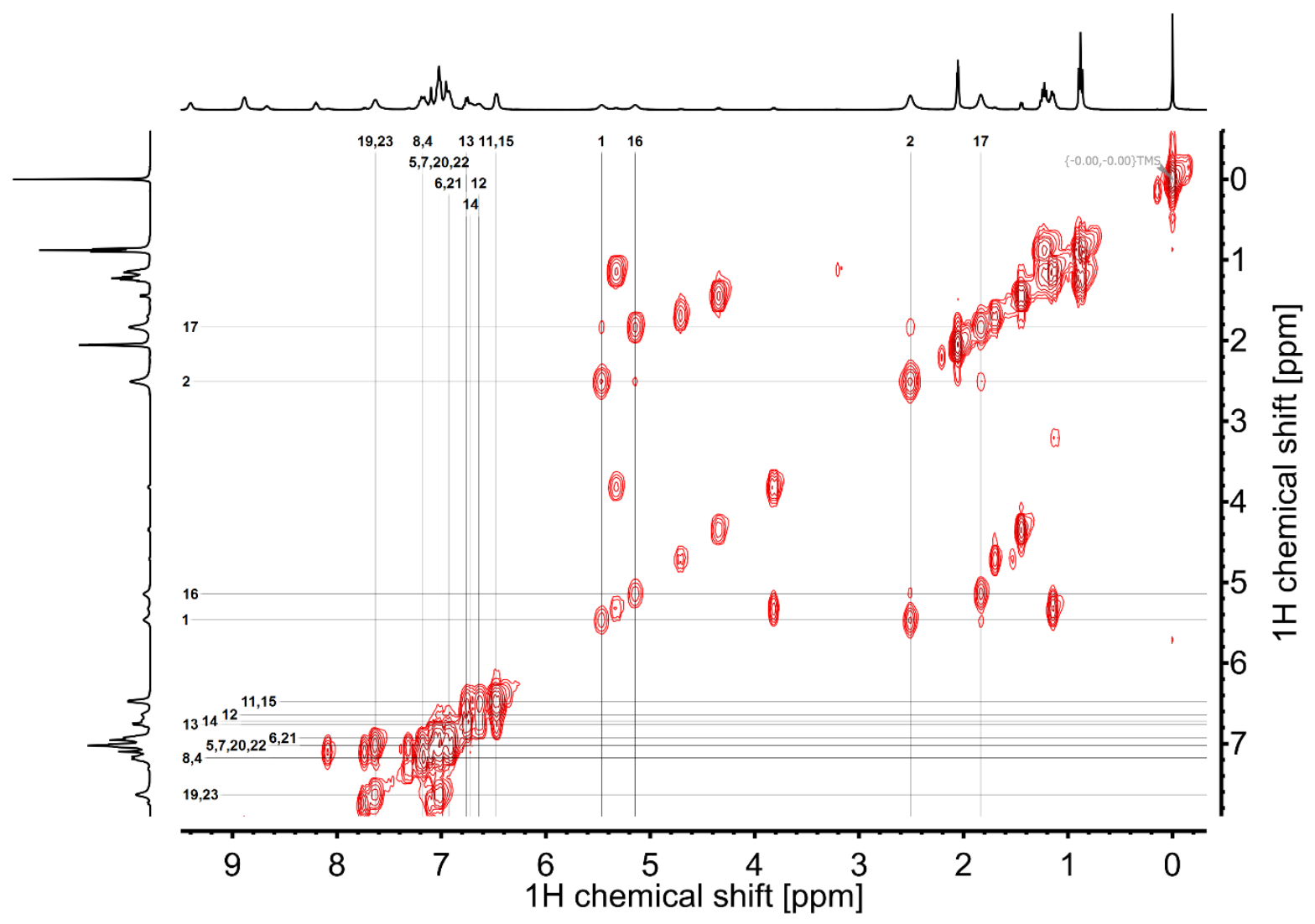

Figure S23. ${ }^{1} \mathrm{H}-{ }^{1} \mathrm{H}-\mathrm{COSY}$ spectrum of $\left[\mathrm{CeCl}((S)-\mathrm{PEBA})_{3}\right](4)$ in toluene- $\mathrm{d}_{8}$ at $243 \mathrm{~K}$ with signal assignments. Impurities of $(S)$-HPEBA and $(S)$-LiPEBA also show intense signals.

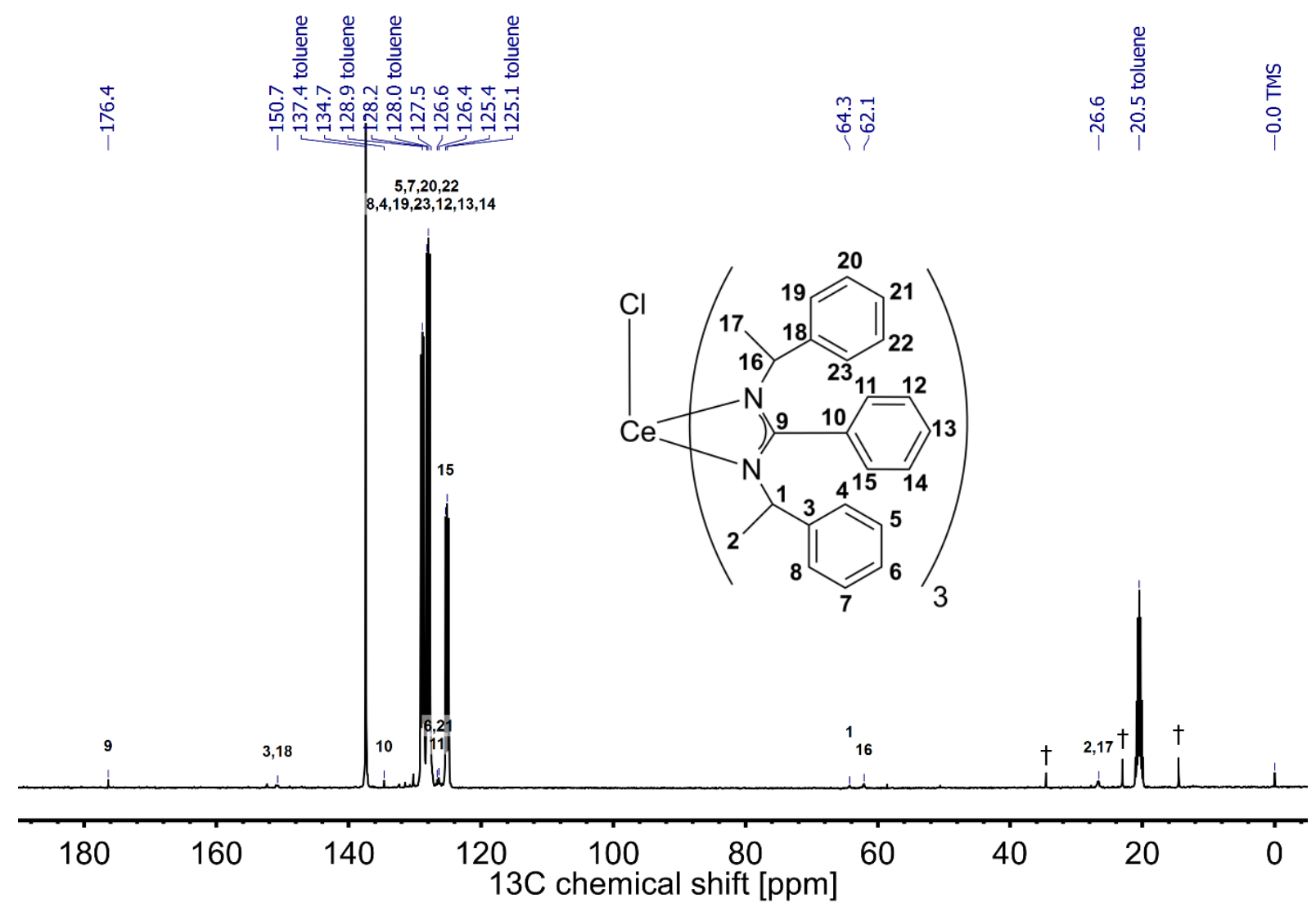

Figure S24. ${ }^{13} \mathrm{C}-\mathrm{NMR}$ spectrum of $\left[\mathrm{CeCl}((S) \text {-PEBA })_{3}\right](4)$ in toluene- $\mathrm{d}_{8}$ at $243 \mathrm{~K}$ with signal assignments. Dagger $(\dagger)$ indicates impurity of $n$-pentane from washing procedure. 


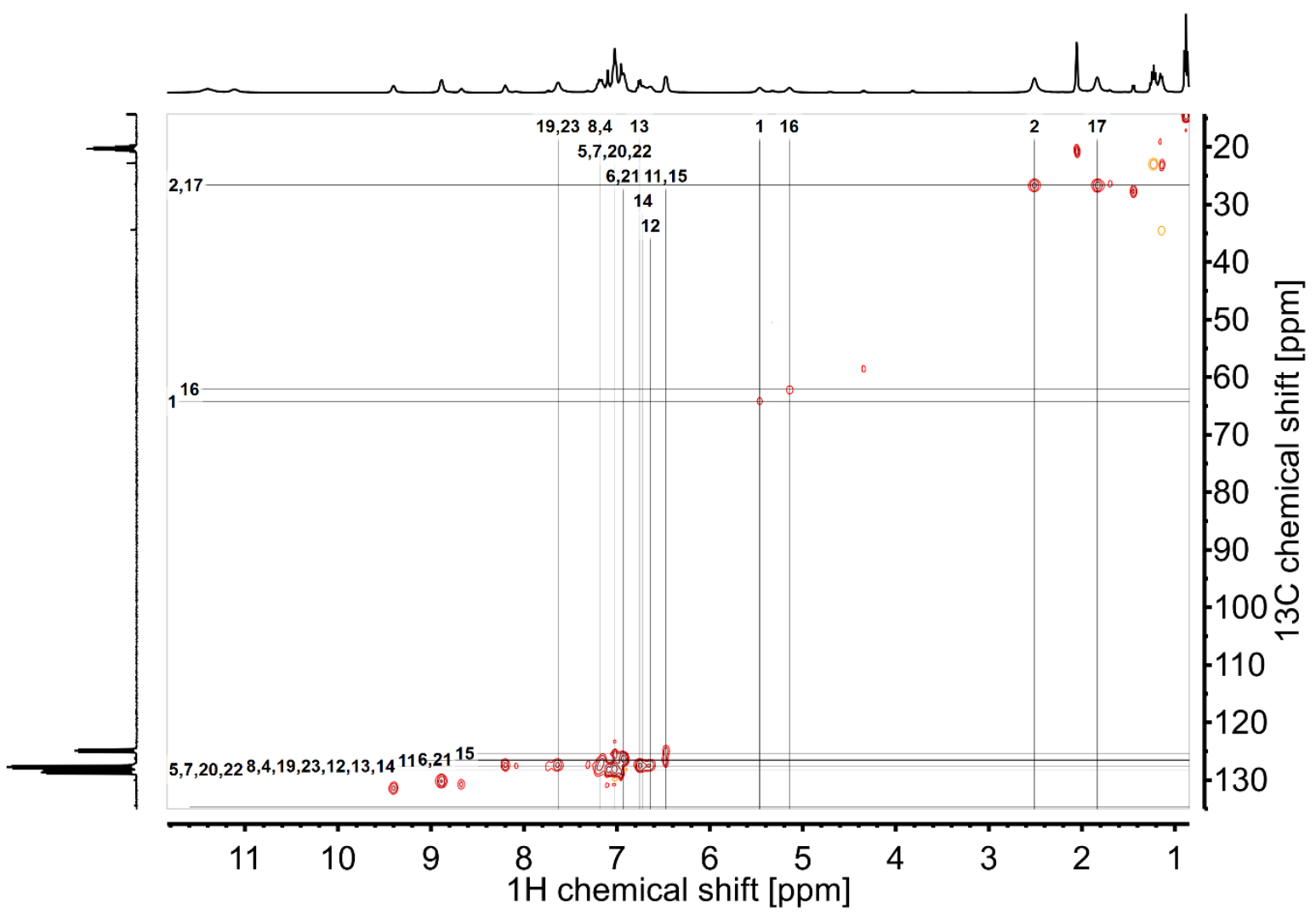

Figure S25. ${ }^{1} \mathrm{H}_{-}{ }^{13} \mathrm{C}-\mathrm{HSQC}$ spectrum of $\left[\mathrm{CeCl}((S)-\mathrm{PEBA})_{3}\right](4)$ in toluene-d $\mathrm{d}_{8}$ at $243 \mathrm{~K}$ with signal assignments.

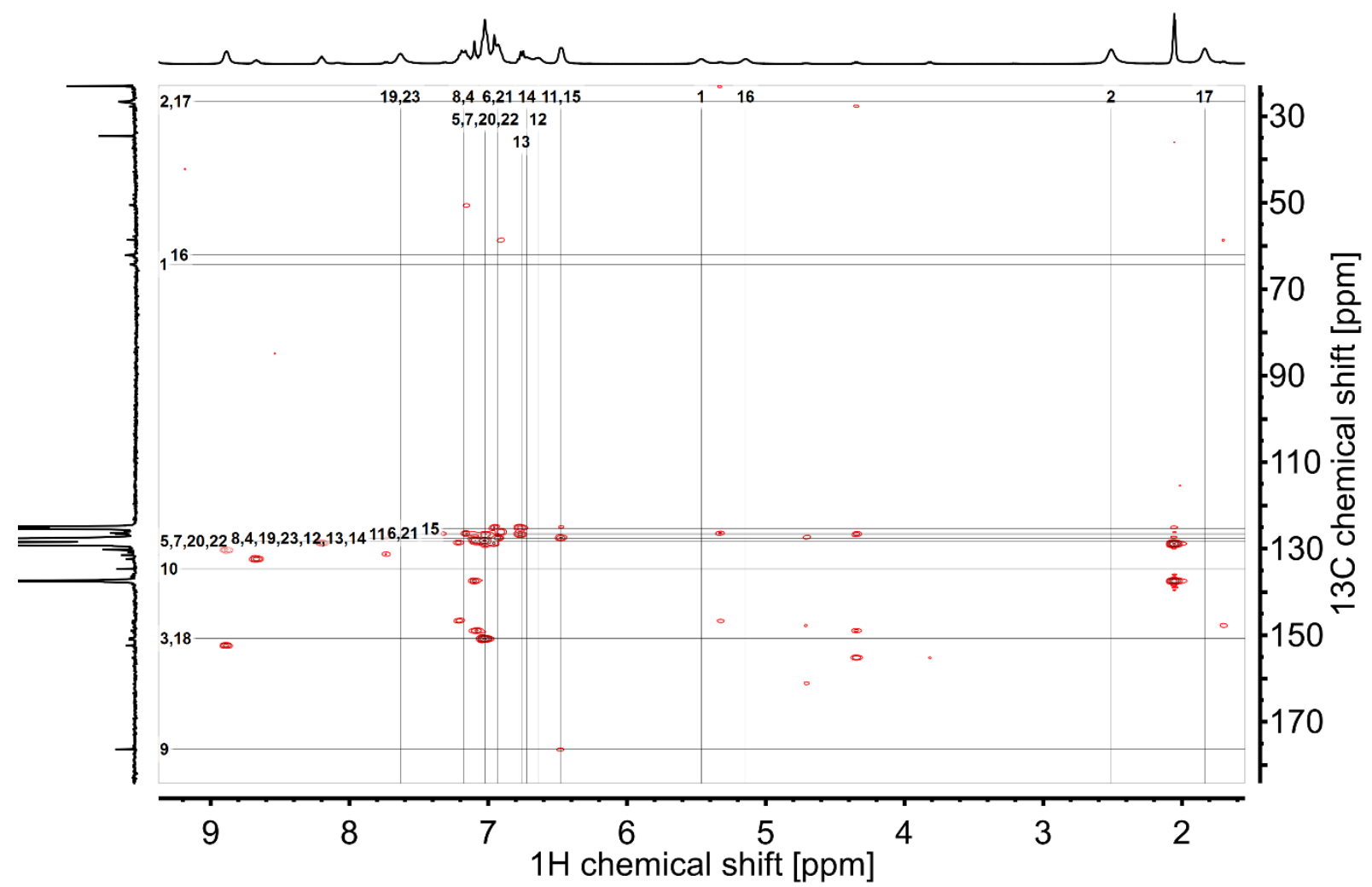

Figure S26. ${ }^{1} \mathrm{H}_{-}{ }^{13} \mathrm{C}-\mathrm{HMBC}$ spectrum of $\left[\mathrm{CeCl}((S)-\mathrm{PEBA})_{3}\right](4)$ in toluene-d $\mathrm{d}_{8}$ at $243 \mathrm{~K}$ with signal assignments. 




Figure S27. ${ }^{1} \mathrm{H}-\mathrm{NMR}$ spectrum of $\left[\mathrm{Ce}((S)-\mathrm{PEBA})_{3}\right](\mathbf{5})$ in toluene- $\mathrm{d}_{8}$ at $243 \mathrm{~K}$ with signal assignments. Dagger $(\dagger)$ indicates impurities of $n$-pentane and grease from synthesis procedure.

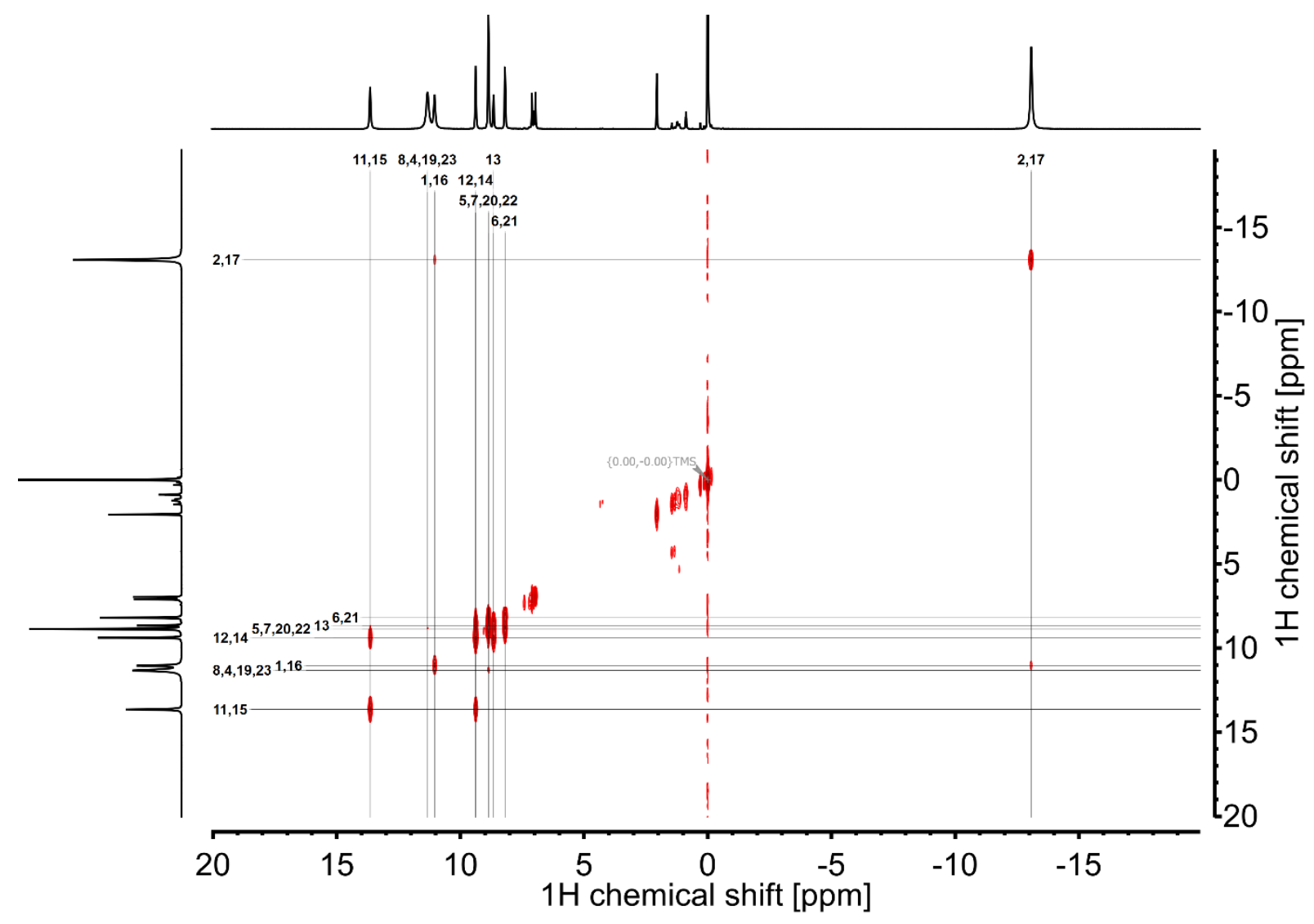

Figure S28. ${ }^{1} \mathrm{H}-{ }^{1} \mathrm{H}-\mathrm{COSY}$ spectrum of $\left[\mathrm{Ce}((S)-\mathrm{PEBA})_{3}\right](5)$ in toluene- $\mathrm{d}_{8}$ at $243 \mathrm{~K}$ with signal assignments. 


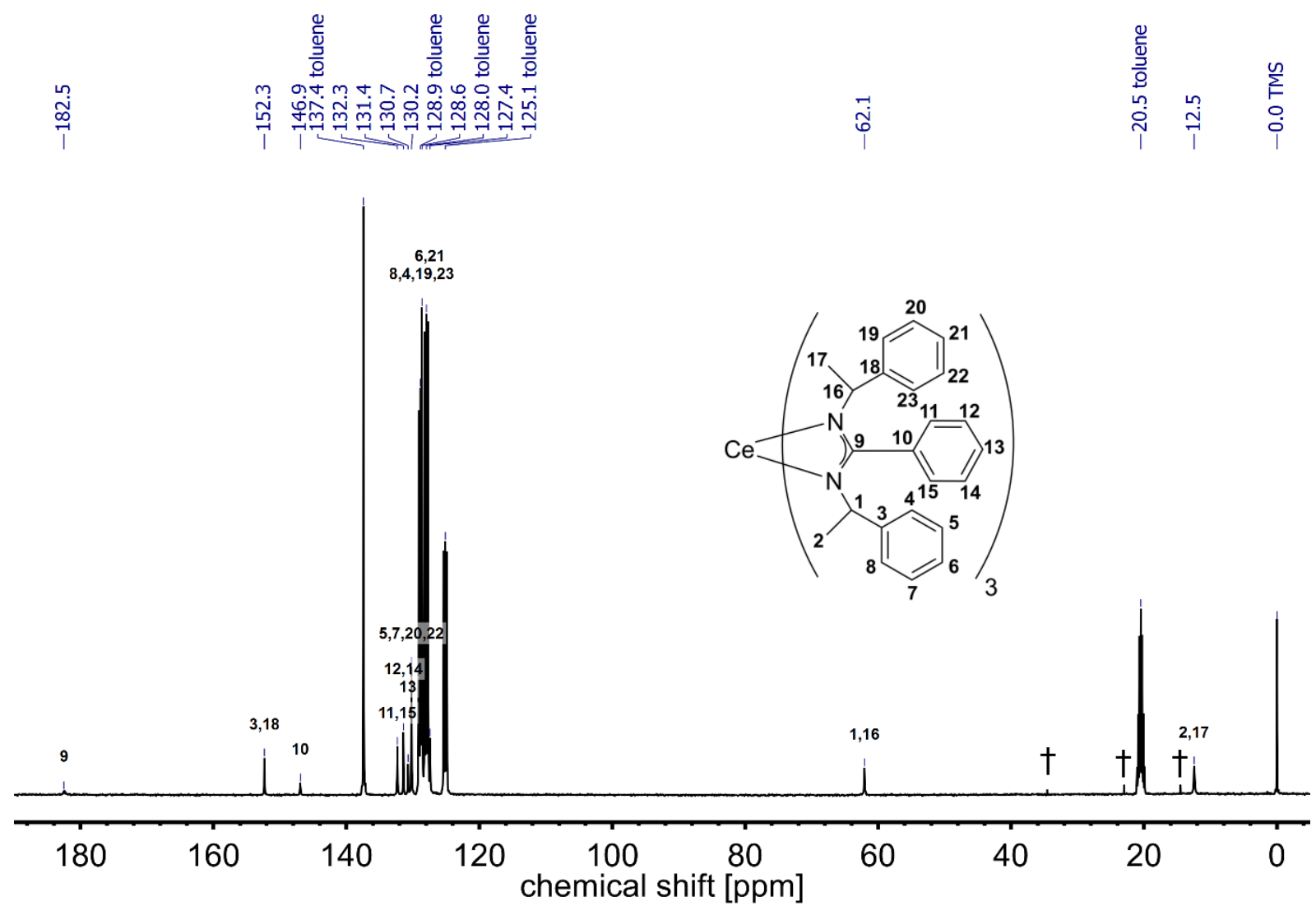

Figure S29. ${ }^{13} \mathrm{C}-\mathrm{NMR}$ spectrum of $\left[\mathrm{Ce}((S) \text {-PEBA })_{3}\right](\mathbf{5})$ in toluene- $\mathrm{d}_{8}$ at $243 \mathrm{~K}$ with signal assignments. Dagger $(\dagger)$ indicates impurity of $n$-pentane from washing procedure.

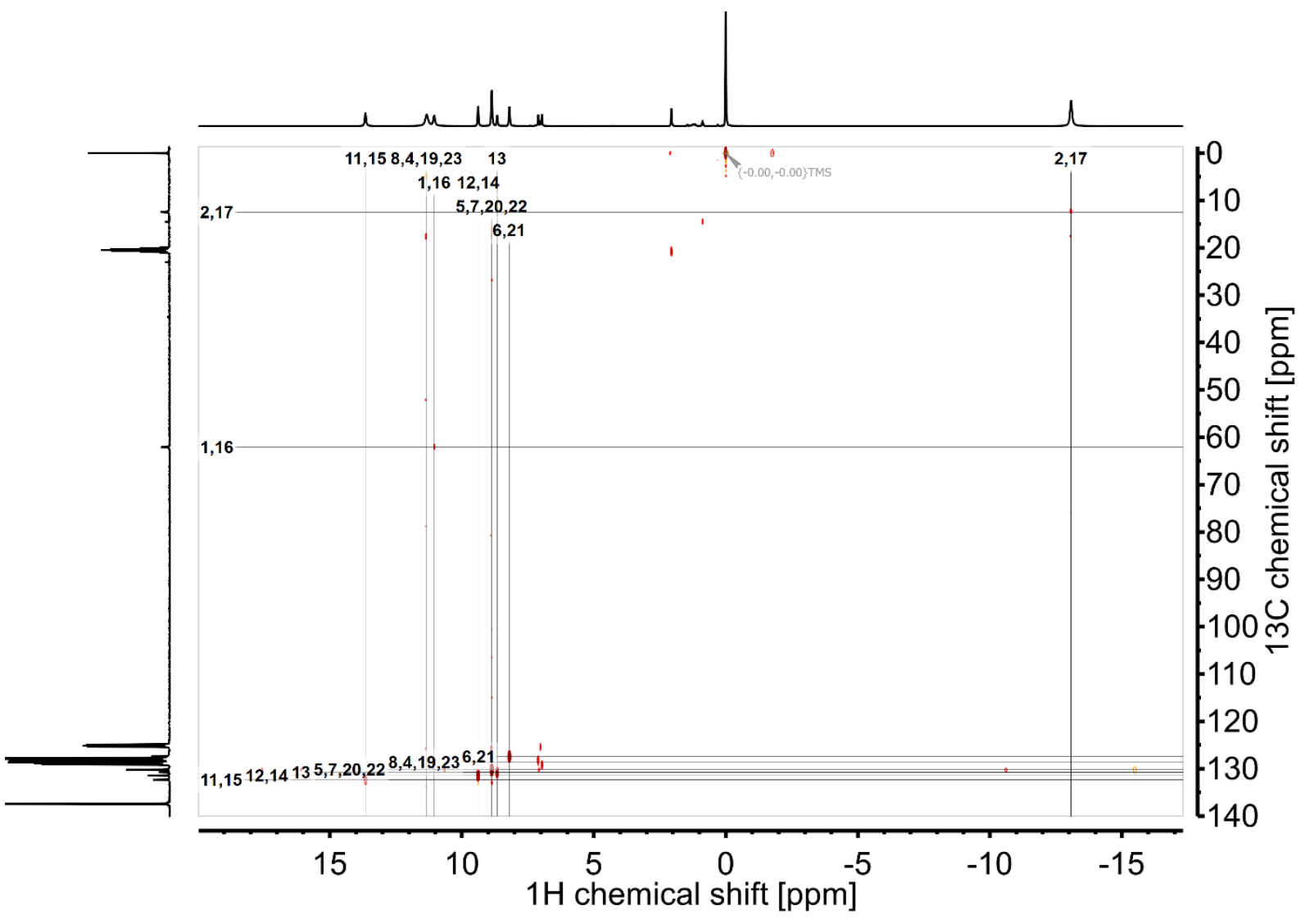

Figure S30. ${ }^{1} \mathrm{H}_{-}{ }^{13} \mathrm{C}-\mathrm{HSQC}$ spectrum of $\left[\mathrm{Ce}((S)-\mathrm{PEBA})_{3}\right](5)$ in toluene-d $\mathrm{d}_{8}$ at $243 \mathrm{~K}$ with signal assignments. 


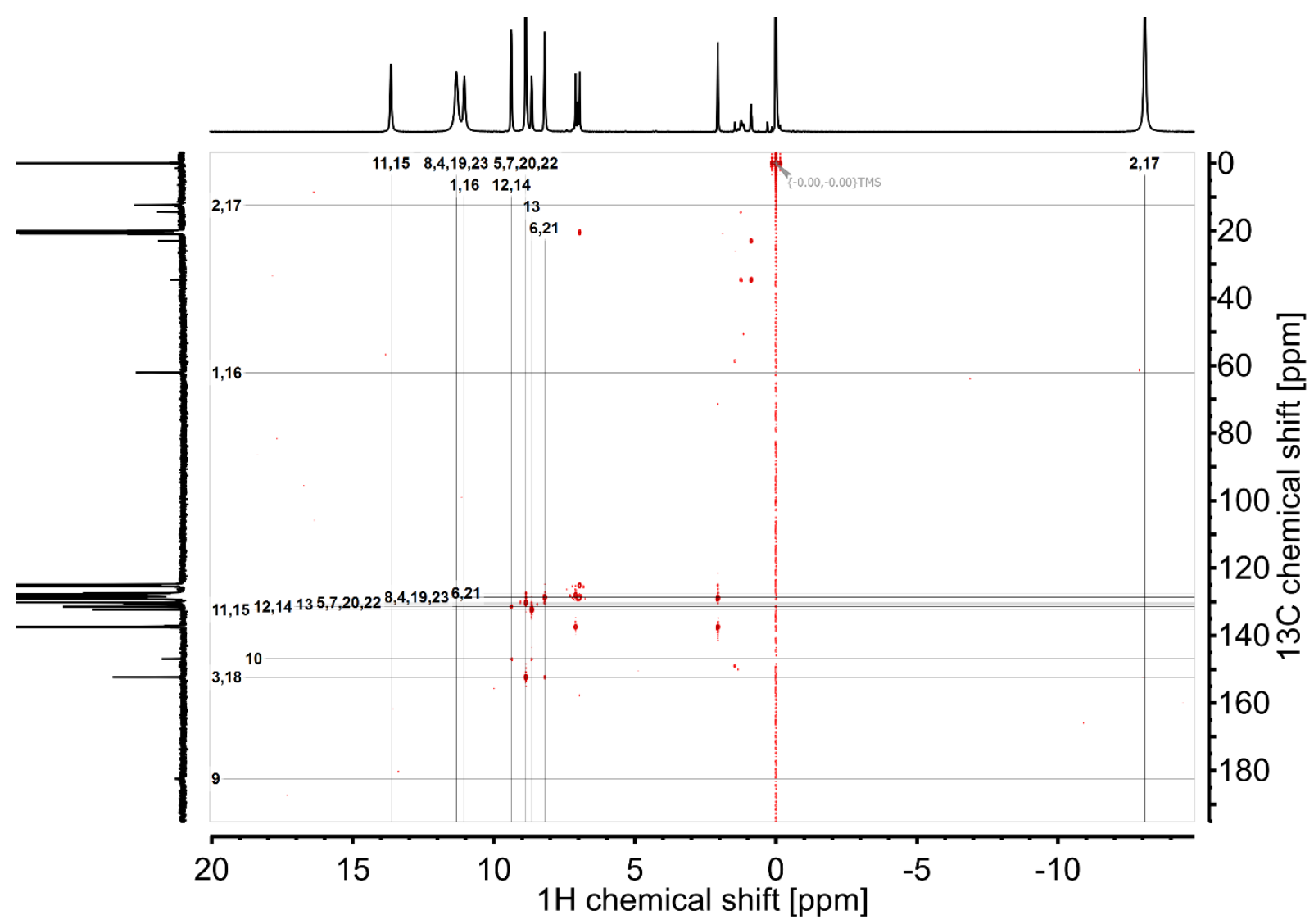

Figure S31. ${ }^{1} \mathrm{H}^{-13} \mathrm{C}-\mathrm{HMBC}$ spectrum of $\left[\mathrm{Ce}((S)-\mathrm{PEBA})_{3}\right](5)$ in toluene- $\mathrm{d}_{8}$ at $243 \mathrm{~K}$ with signal assignments. 


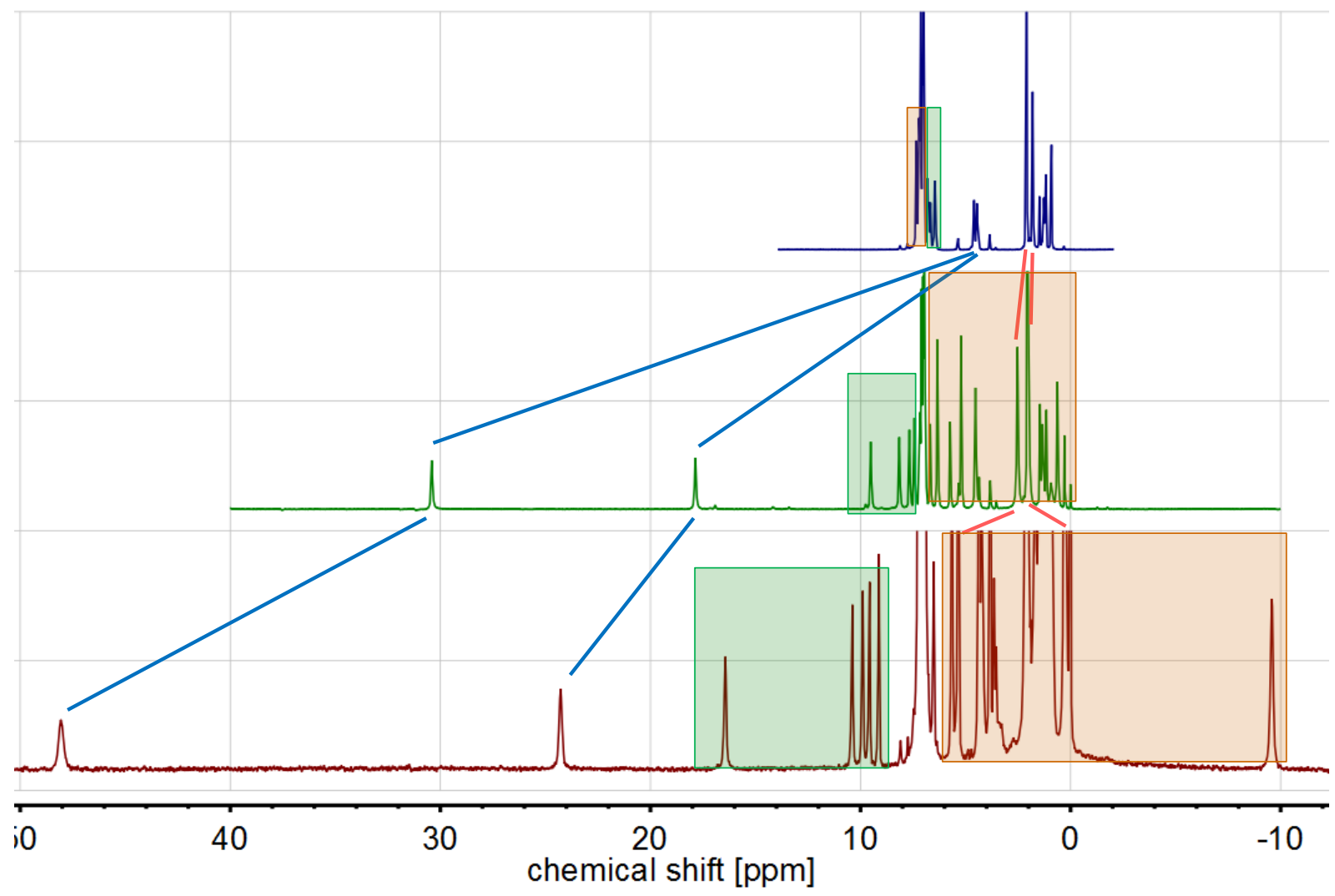

Figure S32. ${ }^{1} \mathrm{H}-\mathrm{NMR}$ spectra of $\left[\mathrm{ThN}_{3}((S)-\mathrm{PEBA})_{3}\right](\mathbf{6}$, top $),\left[\mathrm{UN}_{3}((S)-\mathrm{PEBA})_{3}\right](\mathbf{7}$, middle $),\left[\mathrm{NpN}_{3}((S)-\mathrm{PEBA})_{3}\right]$ $\left(8\right.$, bottom) measured at $243 \mathrm{~K}$ in toluene- $\mathrm{d}_{8}$. Blue lines indicate signal shift for $\mathrm{NCH}$, whereas red lines correspond to methyl groups. Green and brown boxes are dedicated to signals of central (former) and terminal phenyl groups (latter). 


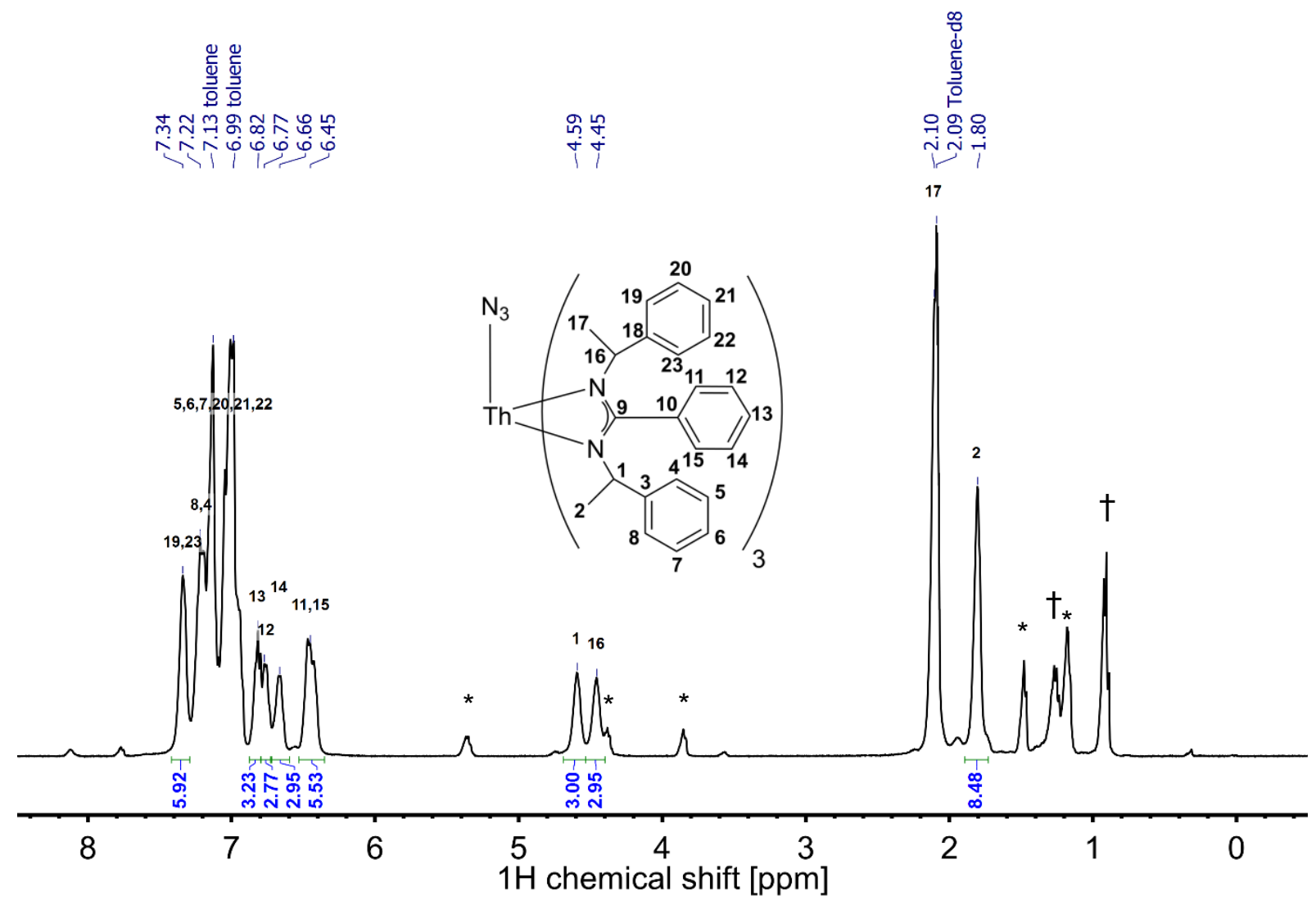

Figure S33. ${ }^{1} \mathrm{H}-\mathrm{NMR}$ spectrum of $\left[\mathrm{ThN}_{3}((S) \text {-PEBA })_{3}\right](6)$ in toluene- $\mathrm{d}_{8}$ at $243 \mathrm{~K}$ with signal assignment. Dagger $(\dagger)$ indicates impurity of $n$-pentane from washing procedure and asterisk $\left(^{*}\right)$ signals of $(S)$-HPEBA as small impurity.

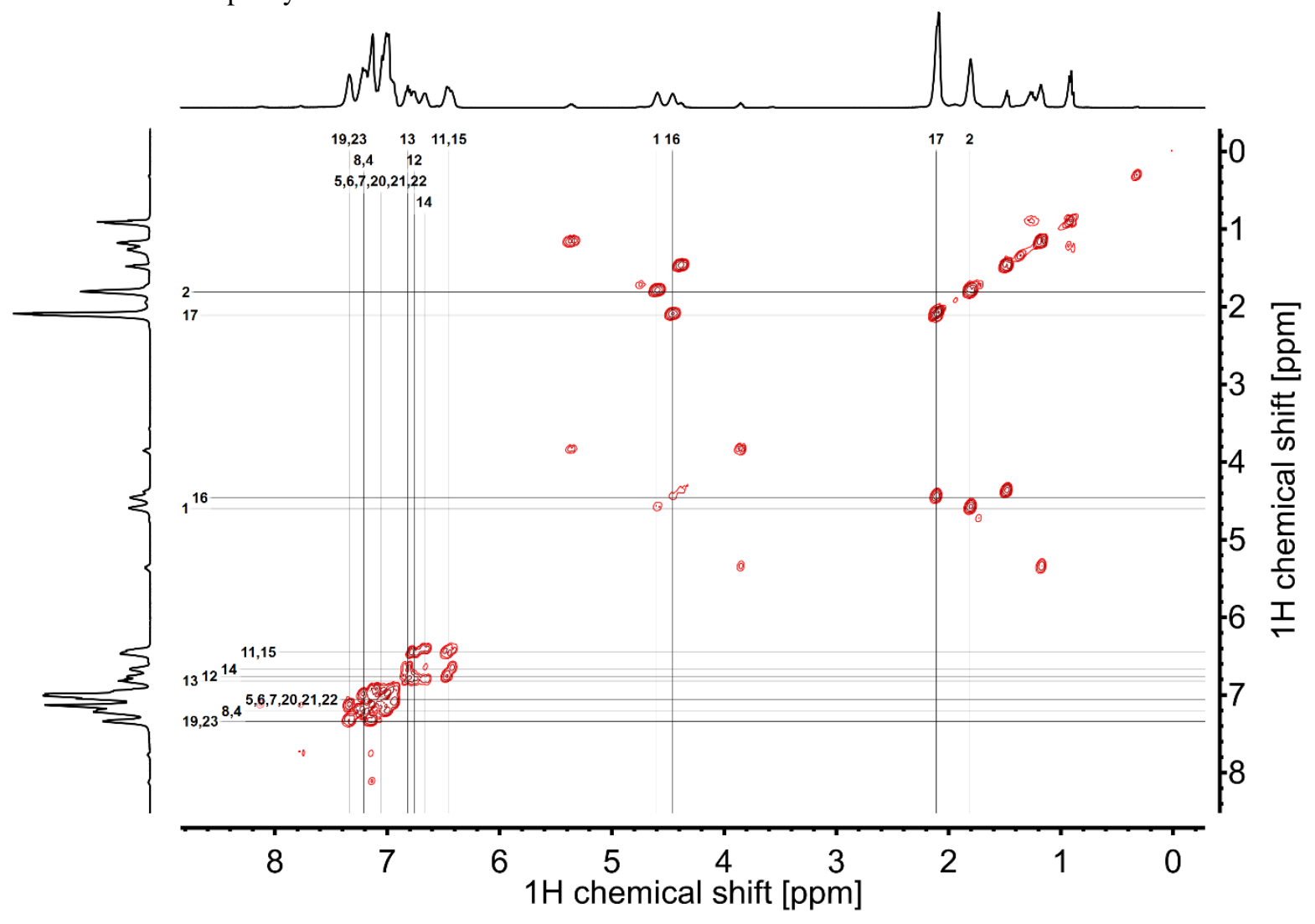

Figure S34. ${ }^{1} \mathrm{H}-{ }^{1} \mathrm{H}-\mathrm{COSY}$ spectrum of $\left[\mathrm{ThN}_{3}((S) \text {-PEBA })_{3}\right](\mathbf{6})$ in toluene- $\mathrm{d}_{8}$ at $243 \mathrm{~K}$ with signal assignments. Impurity of $(S)$-HPEBA also show intense signals. 


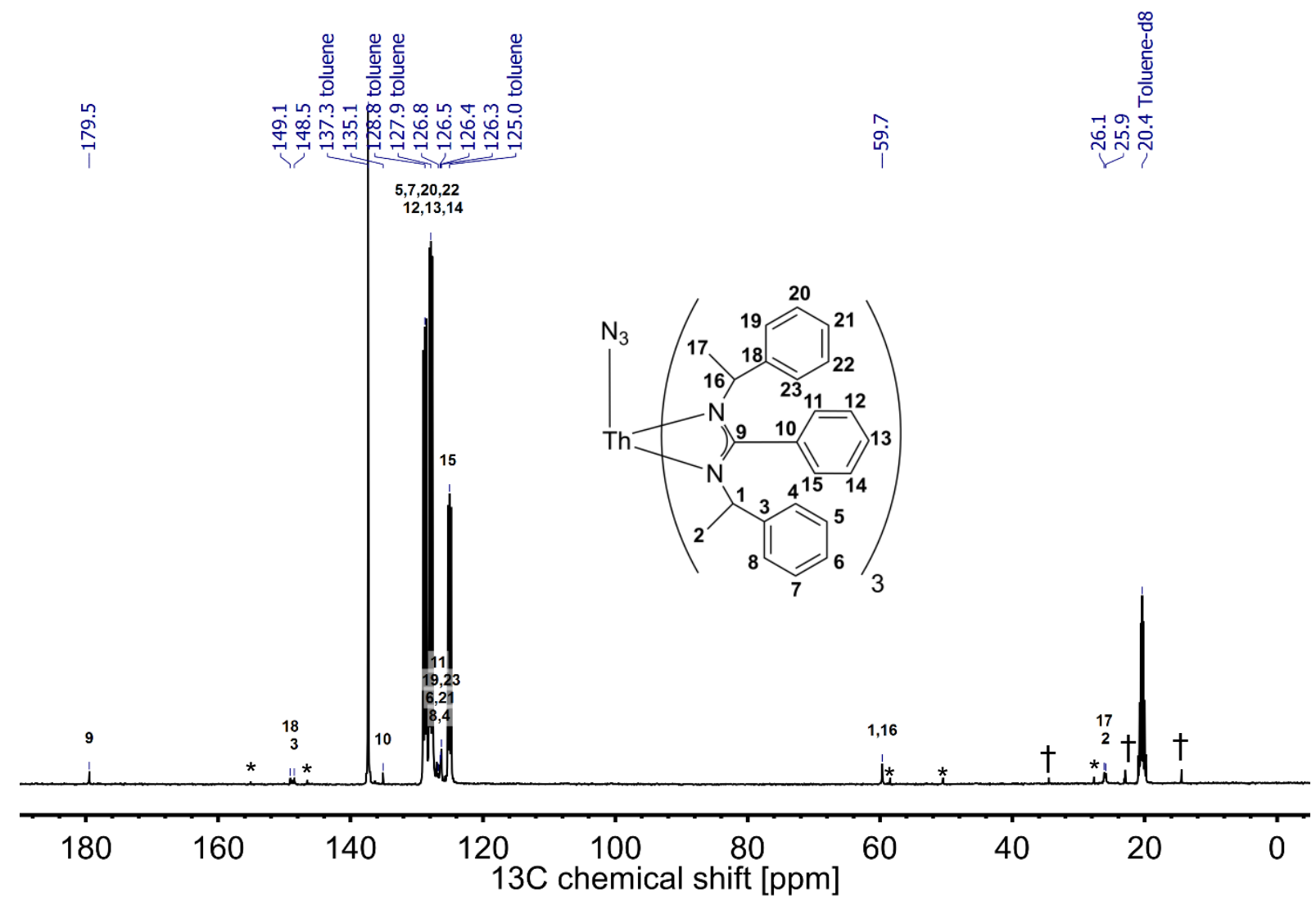

Figure S35. ${ }^{13} \mathrm{C}-\mathrm{NMR}$ spectrum of $\left[\mathrm{ThN}_{3}((S)-\mathrm{PEBA})_{3}\right](\mathbf{6})$ in toluene- $\mathrm{d}_{8}$ at $243 \mathrm{~K}$ with signal assignments. Dagger $(\dagger)$ indicates impurity of $n$-pentane from washing procedure and asterisk $(*)$ signals of $(S)$-HPEBA as small impurity.

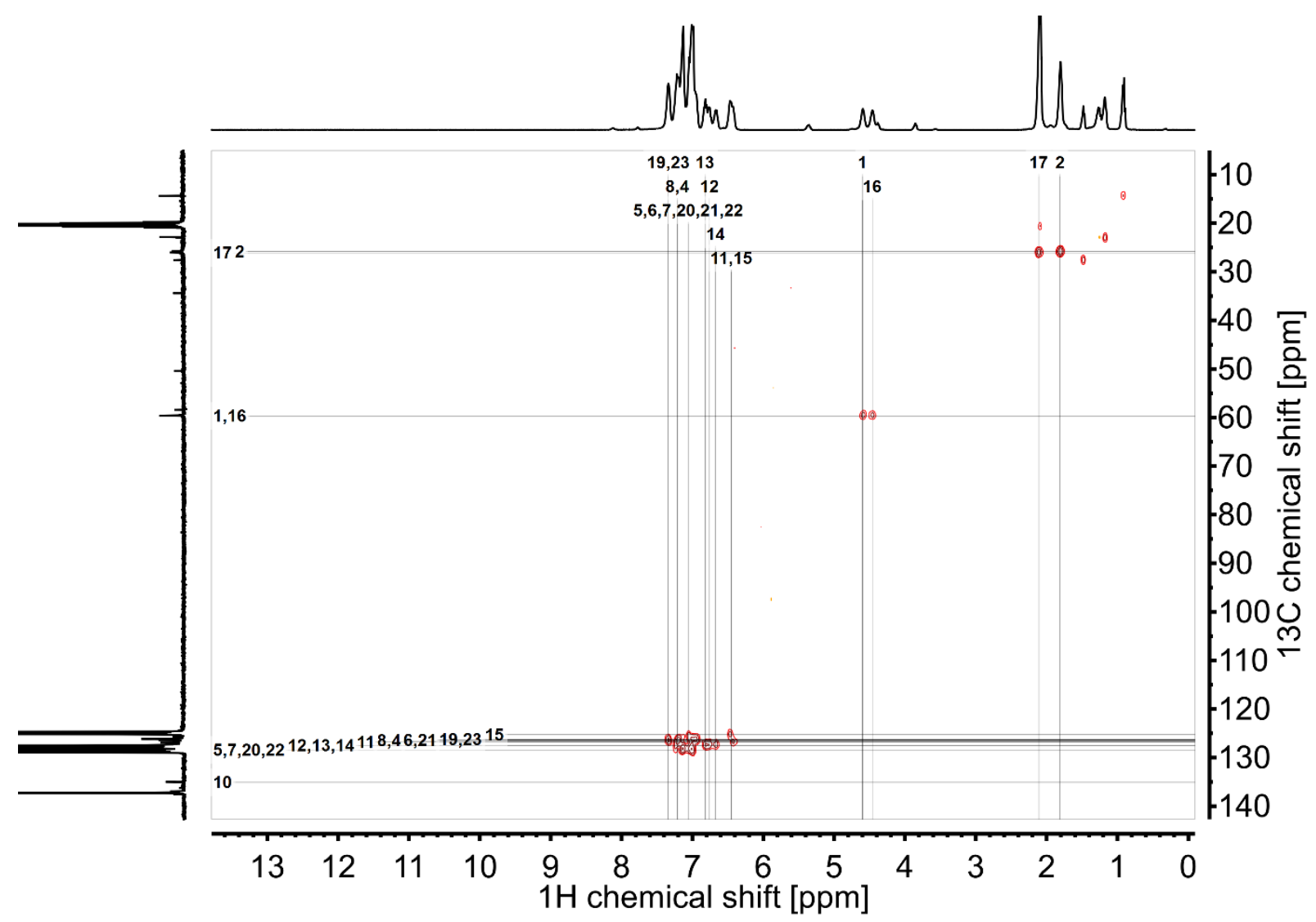

Figure S36. ${ }^{1} \mathrm{H}_{-}{ }^{13} \mathrm{C}-\mathrm{HSQC}$ spectrum of $\left[\mathrm{ThN}_{3}((S)-\mathrm{PEBA})_{3}\right](6)$ in toluene- $\mathrm{d}_{8}$ at $243 \mathrm{~K}$ with signal assignments. 


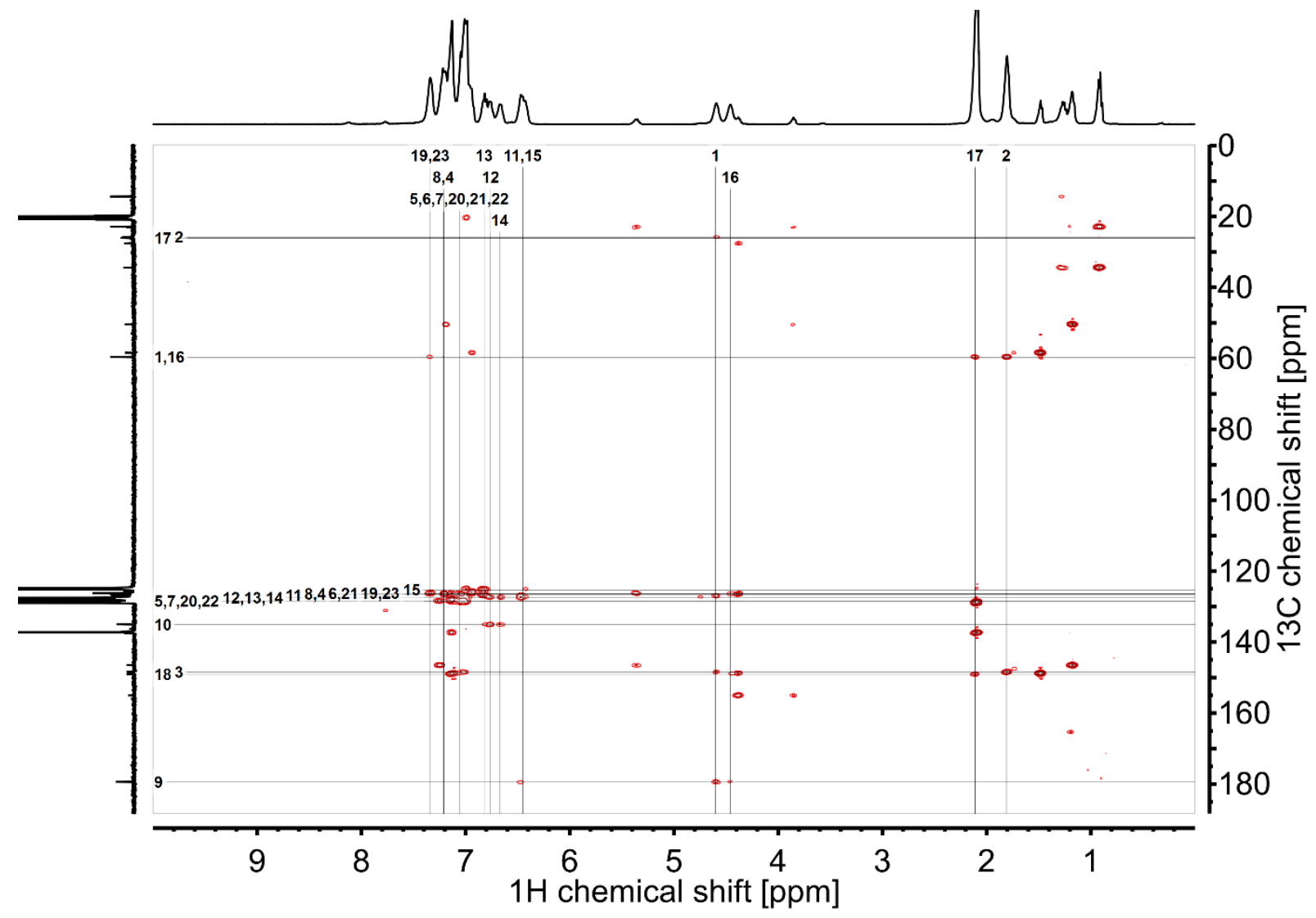

Figure S37. ${ }^{1} \mathrm{H}-{ }^{13} \mathrm{C}-\mathrm{HMBC}$ spectrum of $\left[\mathrm{ThN}_{3}((S)-\mathrm{PEBA})_{3}\right](6)$ in toluene- $\mathrm{d}_{8}$ at $243 \mathrm{~K}$ with signal assignments. Impurity of $(S)$-HPEBA also show intense signals. 


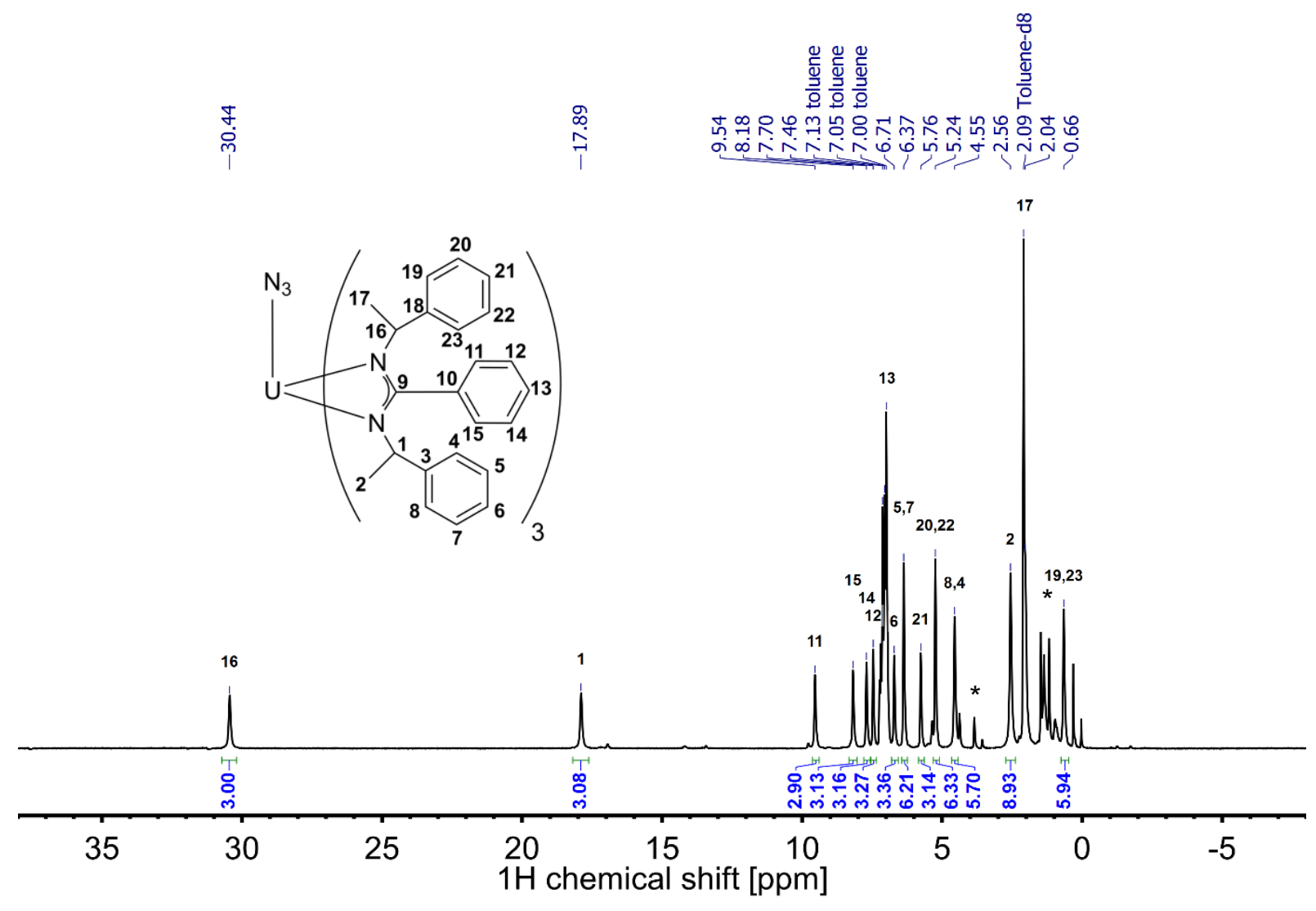

Figure S38. ${ }^{1} \mathrm{H}-\mathrm{NMR}$ spectrum of $\left[\mathrm{UN}_{3}((S)-\mathrm{PEBA})_{3}\right](7)$ in toluene- $\mathrm{d}_{8}$ at $243 \mathrm{~K}$ with signal assignments. Asterisk $(*)$ indicates small impurity of $(S)$-HPEBA.

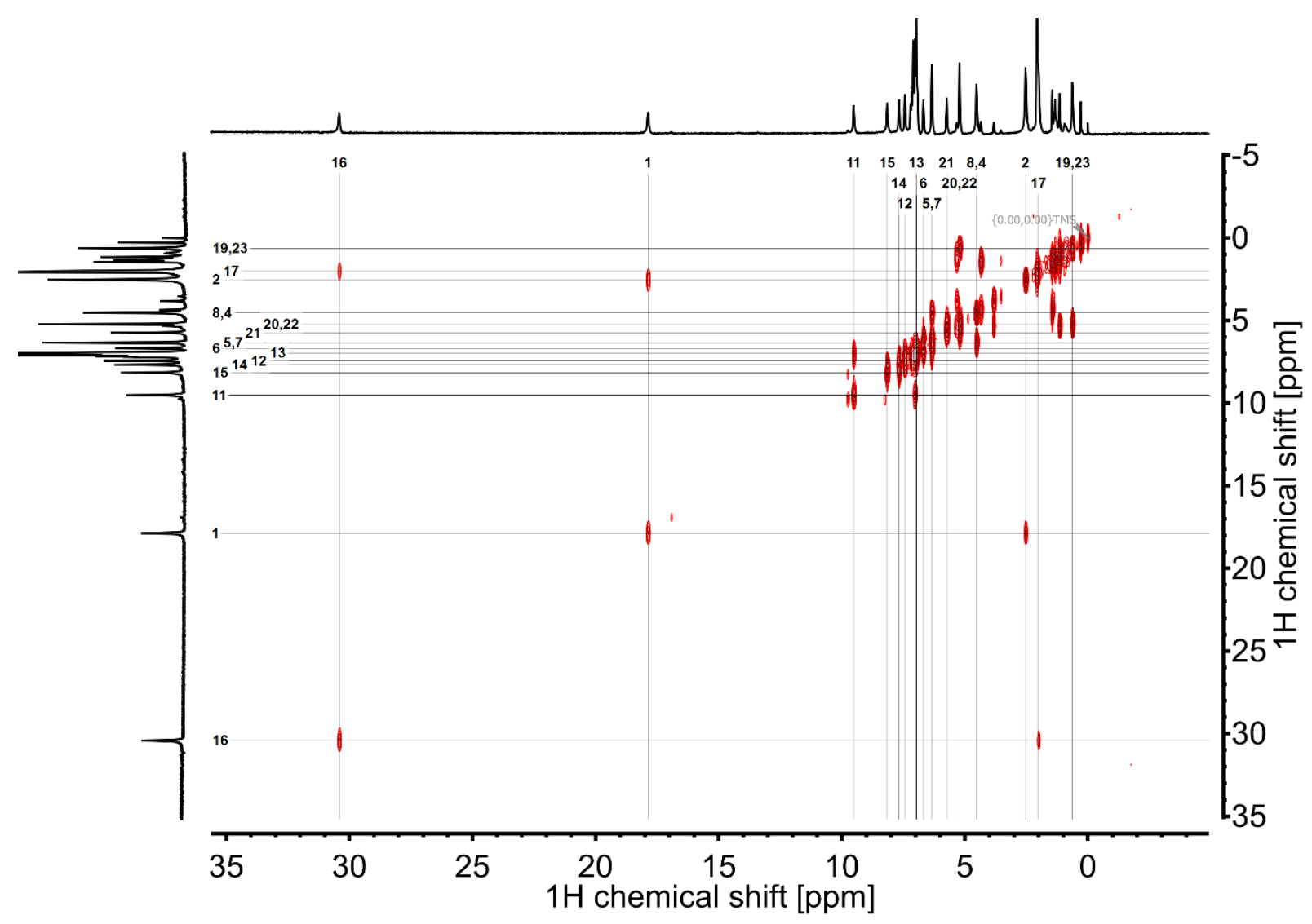

Figure S39. ${ }^{1} \mathrm{H}-{ }^{1} \mathrm{H}-\mathrm{COSY}$ spectrum of $\left[\mathrm{UN}_{3}((S)-\mathrm{PEBA})_{3}\right](7)$ in toluene-d $\mathrm{d}_{8}$ at $243 \mathrm{~K}$ with signal assignments. 


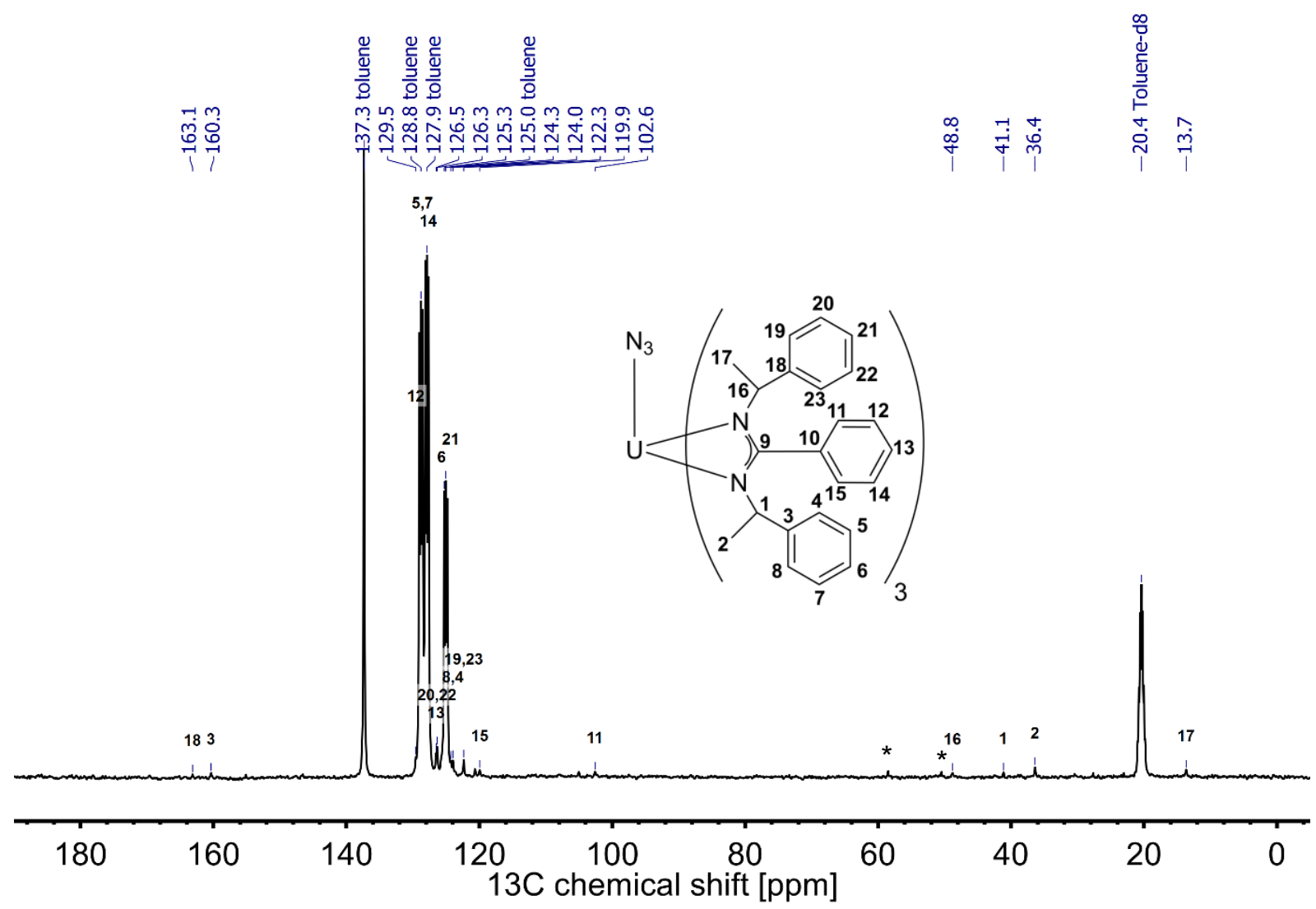

Figure S40. ${ }^{13} \mathrm{C}-\mathrm{NMR}$ spectrum of $\left[\mathrm{UN}_{3}((S)-\mathrm{PEBA})_{3}\right](7)$ in toluene-d $\mathrm{d}_{8}$ at $243 \mathrm{~K}$ with signal assignments. Asterisk $(*)$ indicates small impurity of $(S)$-HPEBA.

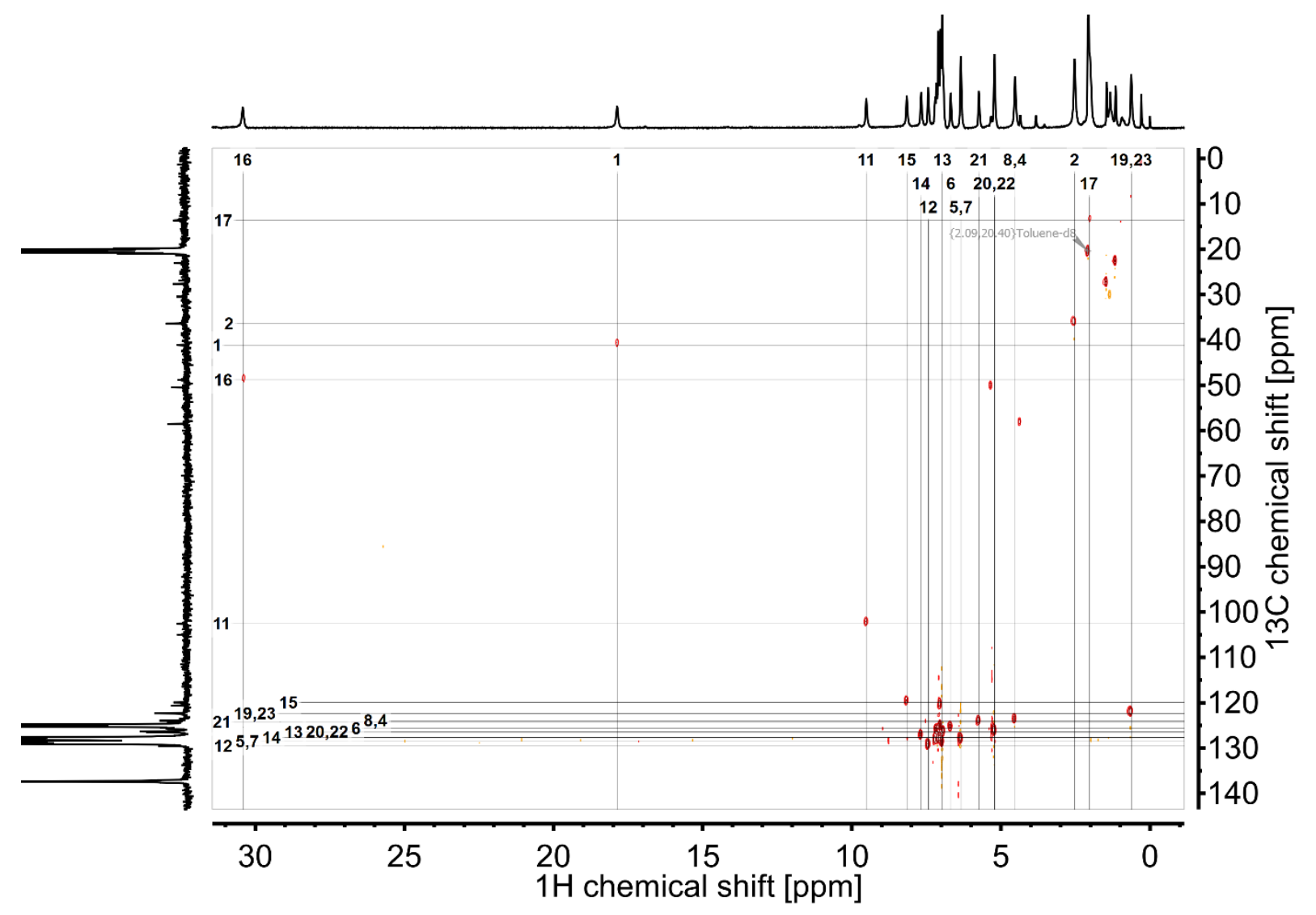

Figure S41. ${ }^{1} \mathrm{H}_{-}{ }^{13} \mathrm{C}-\mathrm{HSQC}$ spectrum of $\left[\mathrm{UN}_{3}((S)-\mathrm{PEBA})_{3}\right](\mathbf{7})$ in toluene-d $\mathrm{d}_{8}$ at $243 \mathrm{~K}$ with signal assignments. 


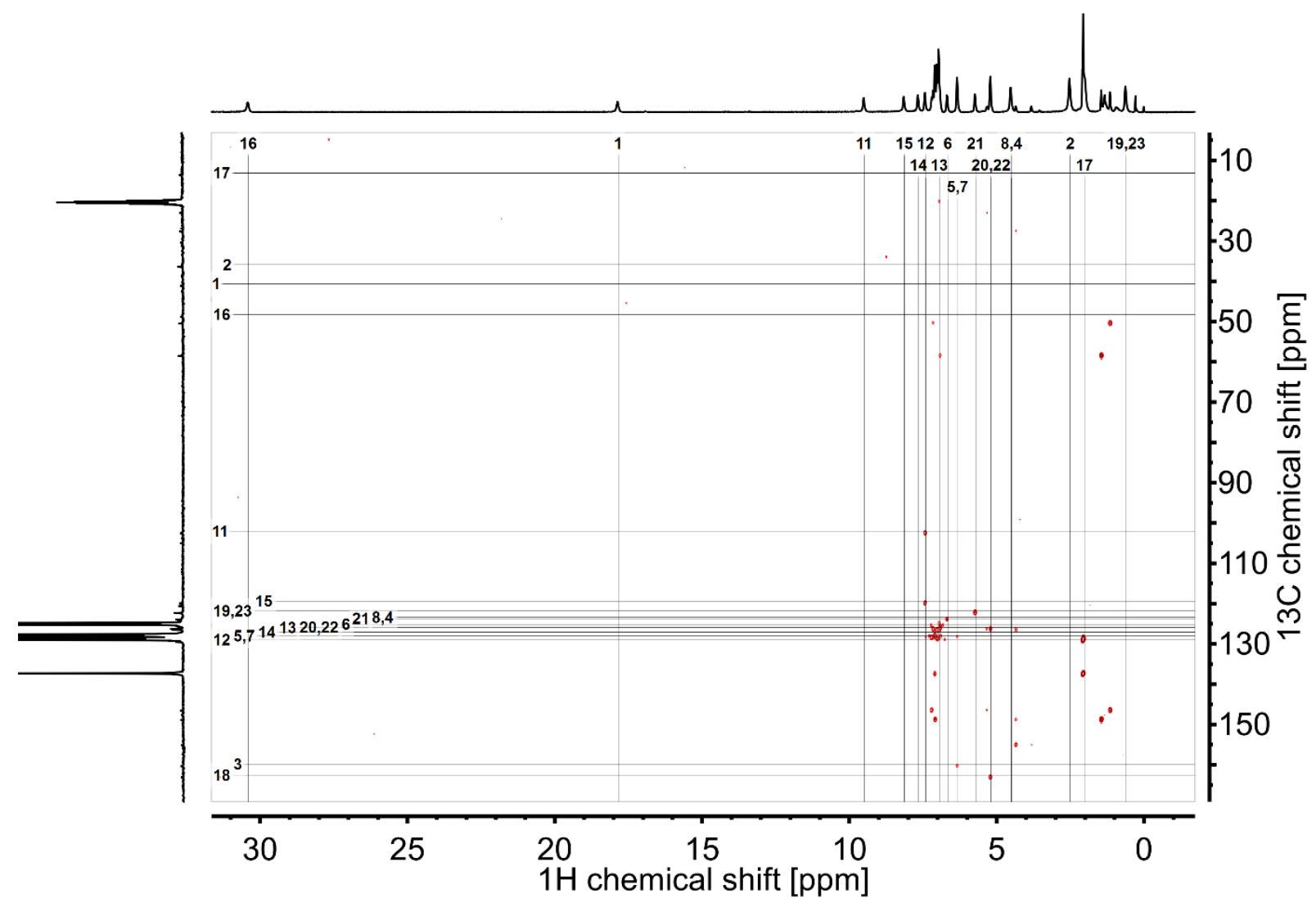

Figure S42. ${ }^{1} \mathrm{H}^{-13} \mathrm{C}-\mathrm{HMBC}$ spectrum of $\left[\mathrm{UN}_{3}((S)-\mathrm{PEBA})_{3}\right](7)$ in toluene-d $\mathrm{d}_{8}$ at $243 \mathrm{~K}$ with signal assignments.

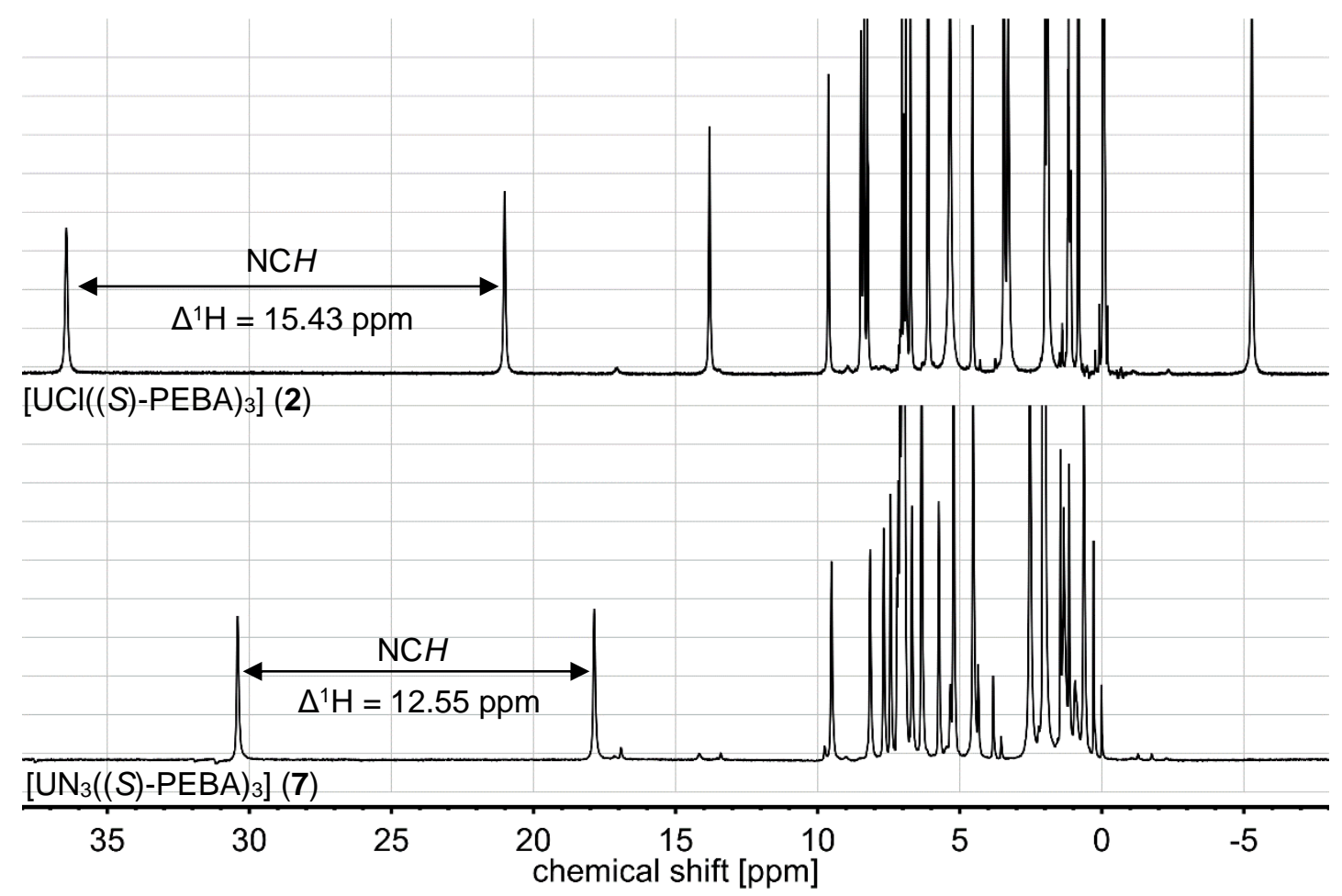

Figure S43. Overlay of ${ }^{1} \mathrm{H}-\mathrm{NMR}$ spectrum of $\left[\mathrm{UCl}((S)-\mathrm{PEBA})_{3}\right](\mathbf{2}$, top $)$ and $\left[\mathrm{UN}_{3}((S)-\mathrm{PEBA})_{3}\right](\mathbf{7}$, bottom $)$ in toluene- $\mathrm{d}_{8}$ at $243 \mathrm{~K}$. Signals of $\mathrm{NCH}$ are highlighted. Other signals are not indicated for clarity. 


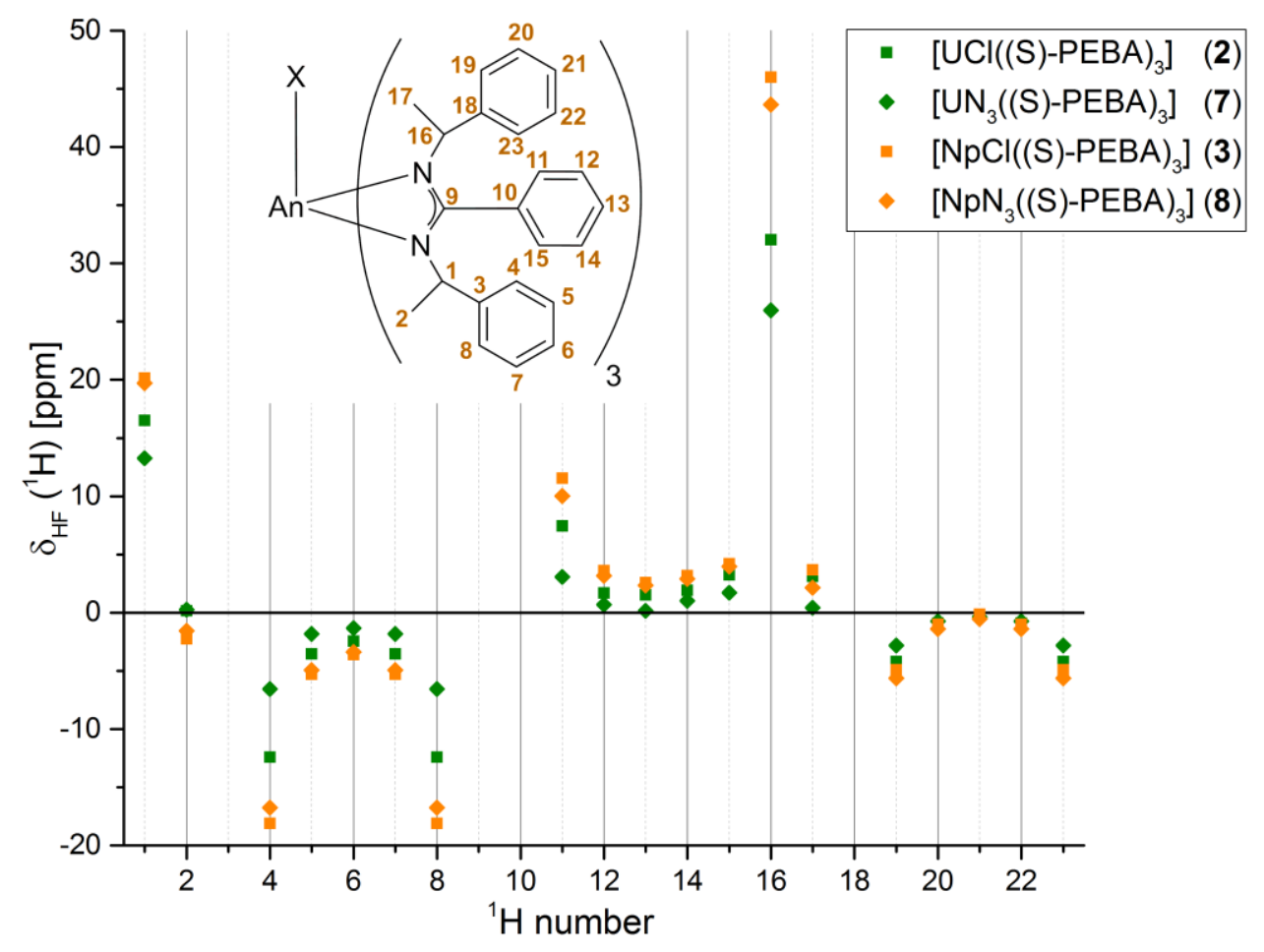

Figure S44. Plot of the ${ }^{1} \mathrm{H}$ hyperfine shift for compounds $2,3,7$, and 8 . The hyperfine shift has been calculated by subtracting the diamagnetic ${ }^{1} \mathrm{H}$ NMR shift of the corresponding thorium complex $\mathbf{1}(\mathrm{Cl})$ or $\mathbf{6}\left(\mathrm{N}_{3}\right)$ for a certain signal from the respective paramagnetically shifted signal in $\mathbf{2 , 3}, \mathbf{7}$, and $\mathbf{8}$. 


\section{UV/vis/NIR spectra}

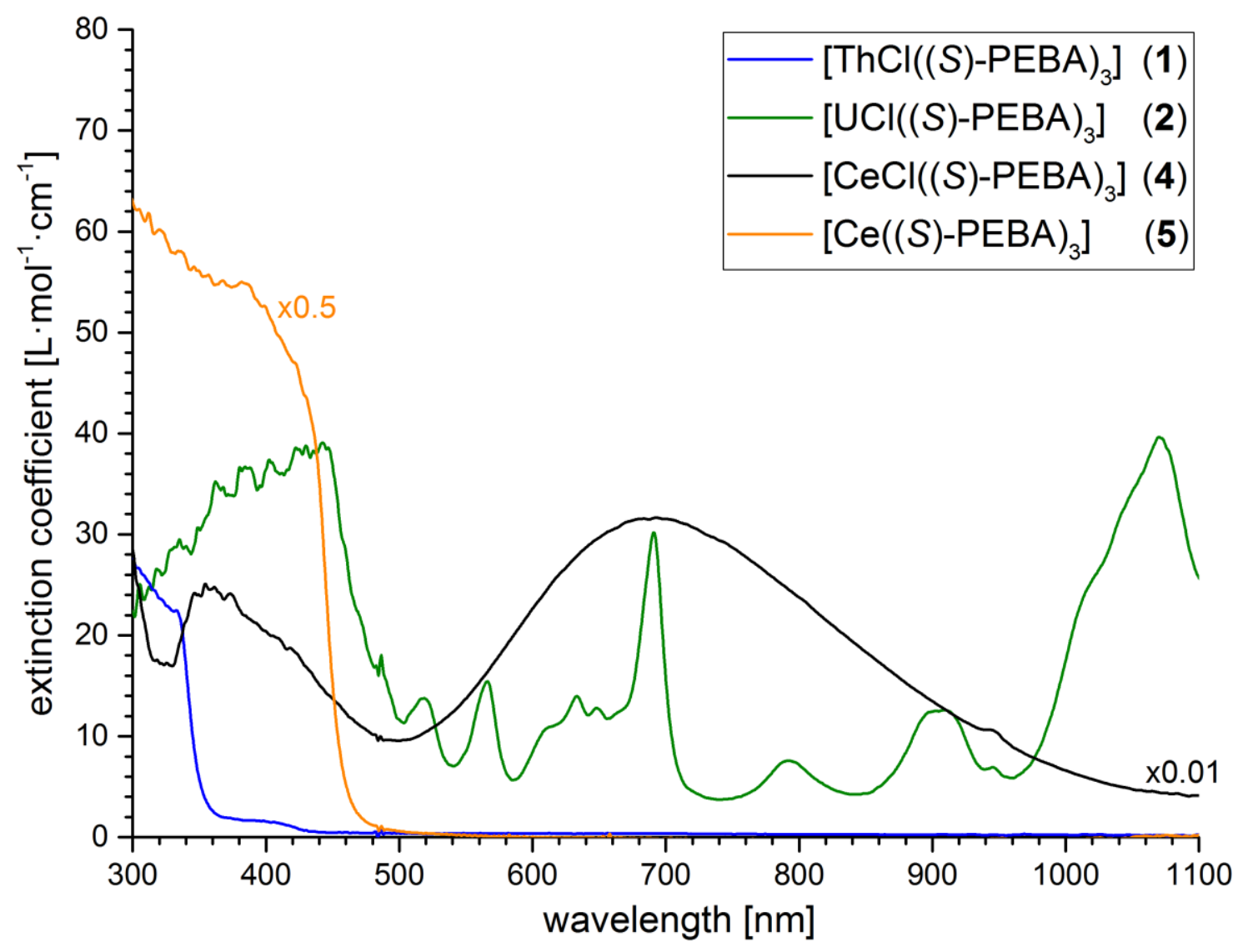

Figure S45. UV/vis/NIR spectra of $f$ element complexes 1, 2, 3, and 4. The spectrum of the cerium complex 4 has been scaled by a factor of 0.01 and the spectrum of 5 by 0.5 .

The UV/vis/NIR spectrum of the uranium complex 2 shows narrow signals in the range between 500 and $1100 \mathrm{~nm}$. These bands can be attributed to $f-f$ transitions and are similar to reported spectra of tetravalent uranium in aqueous solution ${ }^{19}$ and organic solvents. ${ }^{20,21}$ Especially the band at $1060 \mathrm{~nm}$ which can be attributed to a ${ }^{3} \mathrm{H}_{4} \rightarrow{ }^{3} \mathrm{~F}_{3}$ resp. ${ }^{3} \mathrm{H}_{4} \rightarrow{ }^{3} \mathrm{~F}_{4}$ transition and the band at $690 \mathrm{~nm}\left({ }^{3} \mathrm{H}_{4} \rightarrow{ }^{1} \mathrm{G}_{4} ;{ }^{3} \mathrm{H}_{4} \rightarrow{ }^{3} \mathrm{P}_{0}\right)$ are characteristic for the tetravalent oxidation state of uranium. Due to the absence of $5 f$ electrons in the tetravalent thorium complex 1 no transitions are visible between 500 and $1100 \mathrm{~nm}$. The signals between 300 and $500 \mathrm{~nm}$ are due to $\pi-\pi^{*}$ transitions of the amidinate ligands. In contrast, the tetravalent cerium complex 4 shows a broad and intense absorption with a maximum at $690 \mathrm{~nm}$ which is manifested by the deep blue color of 4 . This band can be assigned to a ligand-to-metal charge transfer (LMCT) band which is characteristic for molecular Ce(IV) compounds. ${ }^{22-24}$ 


\section{IR-spectra}

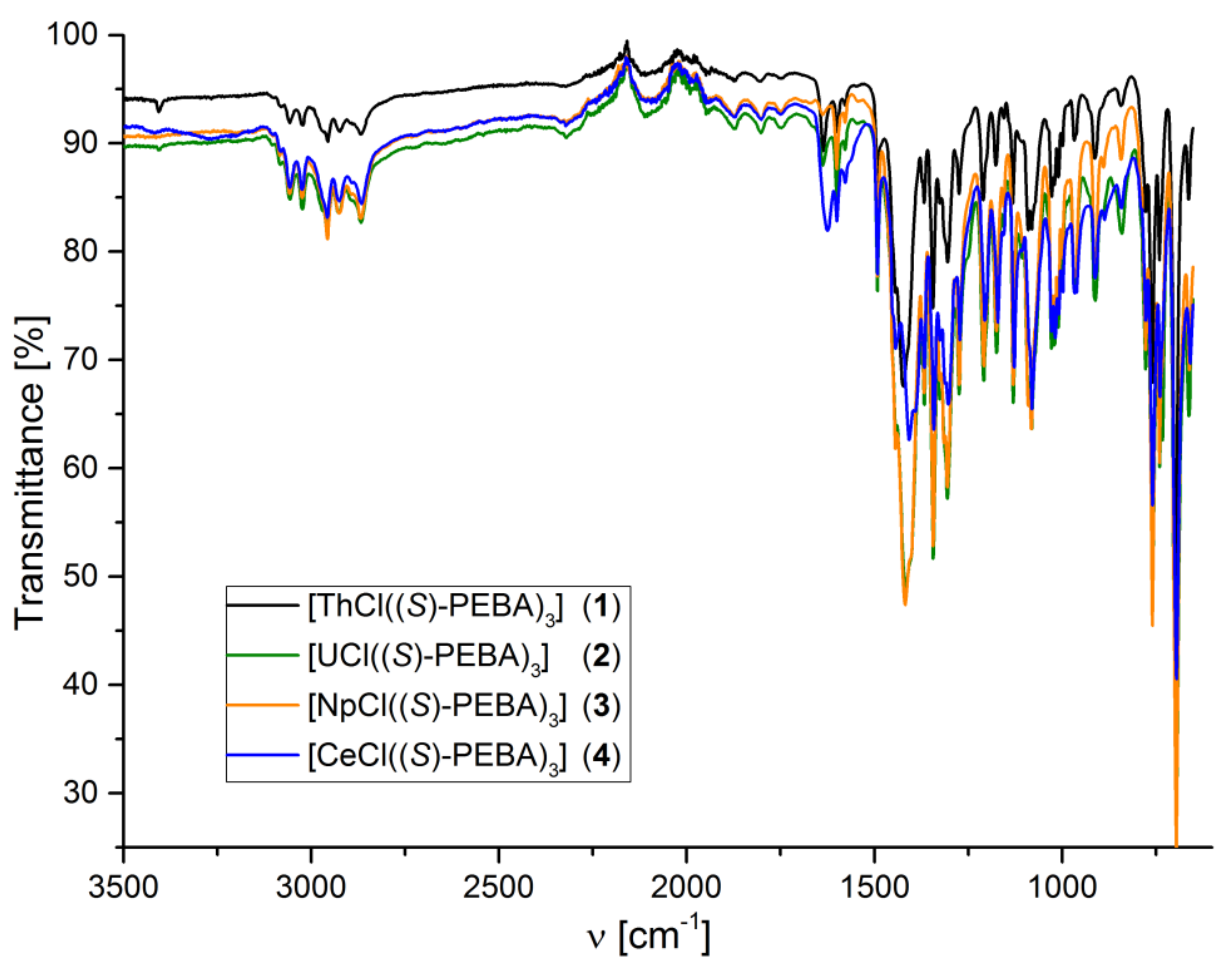

Figure S46. Infrared spectra of isostructural $\left[\mathrm{AnCl}((S)-\mathrm{PEBA})_{3}\right](\mathbf{1 - 4})$ compounds. 


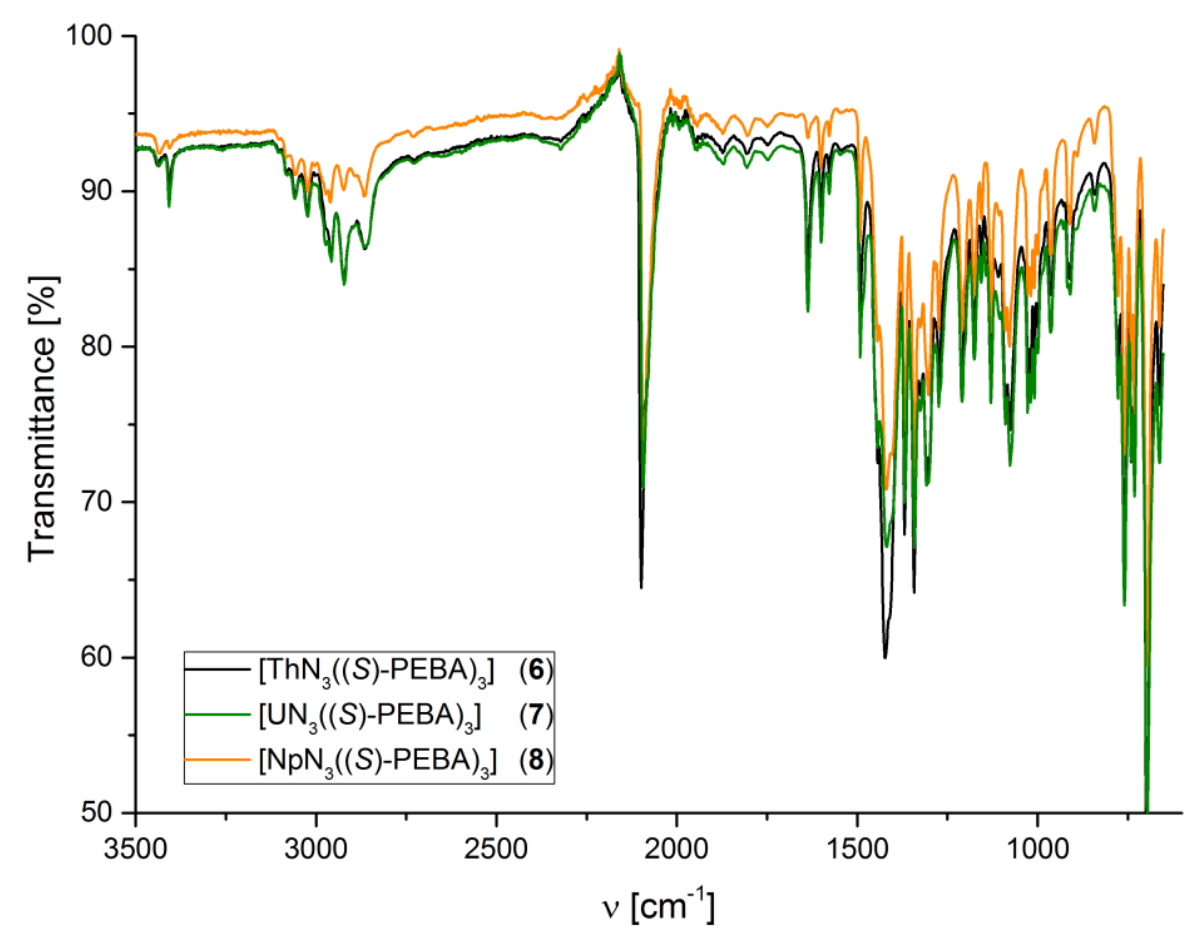

Figure S47. Infrared spectra of isostructural $\left[\mathrm{AnN}_{3}((S)-\mathrm{PEBA})_{3}\right](\mathbf{6 - 8})$ compounds.

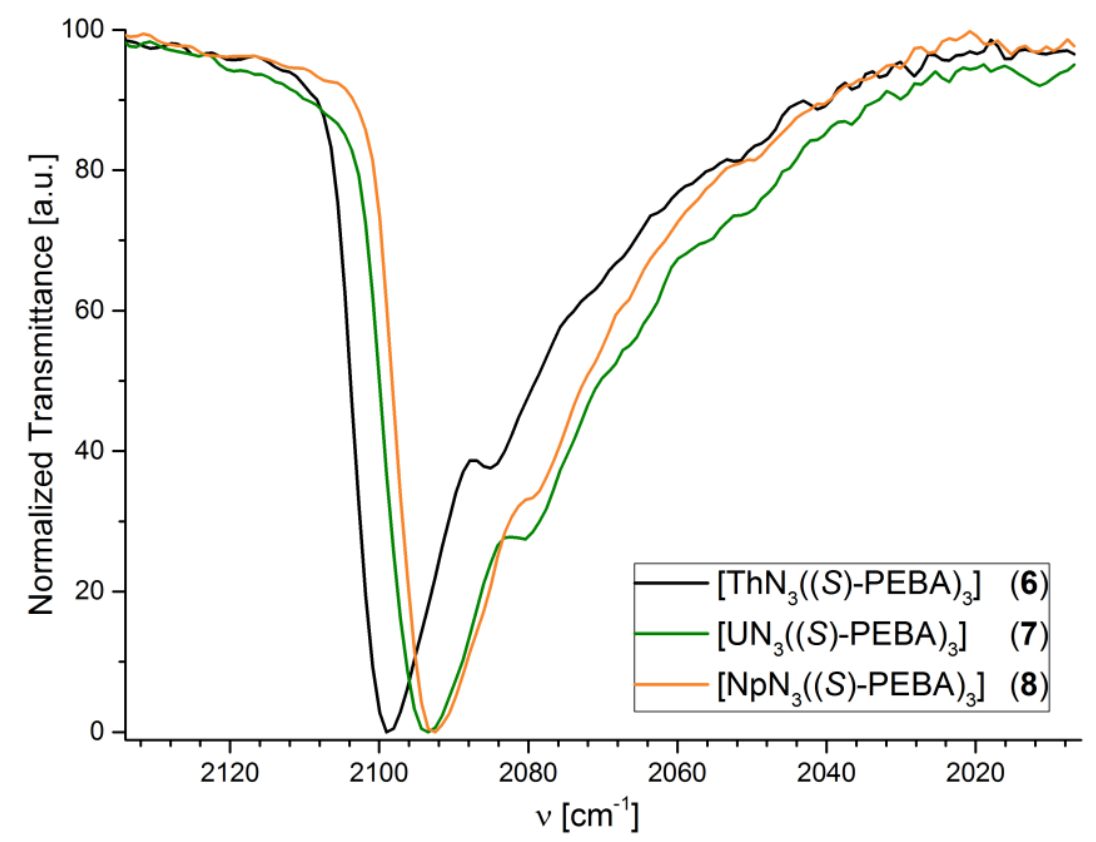

Figure S48. Part of infrared spectra of $\left[\mathrm{AnN}_{3}((S)-\mathrm{PEBA})_{3}\right]$ compounds 6-8 showing the asymmetric stretching mode of the azide group. Spectra were normalized for easier comparison. 


\section{Quantum chemical calculations}

The imaginary wavenumbers produced by frequency analyses within the harmonic approximation are summarized in Table S12. Even after several attempts the authors were not able to reach a minimum on the potential hyper-surface by shifting the structure along the imaginary mode.

Table S12. Imaginary wavenumbers by frequency analysis of optimized complexes.

\begin{tabular}{ll}
\hline complex & Imaginary wavenumbers $\left[\mathrm{cm}^{-1}\right]$ \\
\hline$\left[\mathrm{CeCl}((S)-\mathrm{PEBA})_{3}\right]$ & 0.00 \\
{$\left[\mathrm{ThCl}((S)-\mathrm{PEBA})_{3}\right]$} & 5.13 \\
{$\left[\mathrm{PaCl}((S)-\mathrm{PEBA})_{3}\right]$} & 0.00 \\
{$\left[\mathrm{UCl}((S)-\mathrm{PEBA})_{3}\right]$} & 20.11 \\
{$\left[\mathrm{NpCl}((S)-\mathrm{PEBA})_{3}\right]$} & 17.44 \\
{$\left[\mathrm{PuCl}((S)-\mathrm{PEBA})_{3}\right]$} & 0.00 \\
\hline$\left[\mathrm{CeN}_{3}((S)-\mathrm{PEBA})_{3}\right]$ & 4.75 \\
{$\left[\mathrm{ThN}_{3}((S)-\mathrm{PEBA})_{3}\right]$} & 16.91 \\
{$\left[\mathrm{PaN}_{3}((S)-\mathrm{PEBA})_{3}\right]$} & 0.00 \\
{$\left[\mathrm{UN}_{3}((S)-\mathrm{PEBA})_{3}\right]$} & 32.52 \\
{$\left[\mathrm{NpN}_{3}((S)-\mathrm{PEBA})_{3}\right]$} & 36.97 \\
{$\left[\mathrm{PuN}_{3}((S)-\mathrm{PEBA})_{3}\right]$} & 0.00 \\
\hline
\end{tabular}

The modes possessing imaginary wavenumbers can be assigned to bending motions of the phenyl rings of the ligands. Thus, the obtained structures can be used for further analysis since the wavenumbers are small. Consequently, the ligands are reasonably arranged around the metal centres and the metal-ligand bond-lengths are suitable for comparison. Nevertheless, the authors refrain to compare the calculated IR spectra with the experimental values.

Table S13. Summary of values obtained from QTAIM analysis. Charge $q$ and delocalization index $\delta$ for chloro substituted compounds $\left[\mathrm{MCl}((S)-\mathrm{PEBA})_{3}\right]$.

\begin{tabular}{llllllll}
\hline & $q(\mathrm{M})$ & $q\left(\mathrm{~N}_{\mathrm{a}}\right)$ & $q\left(\mathrm{~N}_{\mathrm{b}}\right)$ & $q(\mathrm{Cl})$ & $\delta\left(\mathrm{M}-\mathrm{N}_{\mathrm{a}}\right)$ & $\delta\left(\mathrm{M}-\mathrm{N}_{\mathrm{b}}\right)$ & $\delta(\mathrm{M}-\mathrm{Cl})$ \\
\hline$\left[\mathrm{CeClL}_{3}\right]$ & +2.44 & -1.21 & -1.21 & -0.69 & 0.40 & 0.48 & 0.54 \\
{$\left[\mathrm{ThClL}_{3}\right]$} & +2.76 & -1.23 & -1.24 & -0.73 & 0.36 & 0.43 & 0.52 \\
{$\left[\mathrm{PaClL}_{3}\right]$} & +2.67 & -1.23 & -1.23 & -0.71 & 0.40 & 0.46 & 0.55 \\
{$\left[\mathrm{UClL}_{3}\right]$} & +2.59 & -1.21 & -1.22 & -0.70 & 0.41 & 0.49 & 0.57 \\
{$\left[\mathrm{NpClL}_{3}\right]$} & +2.53 & -1.22 & -1.22 & -0.70 & 0.41 & 0.49 & 0.58 \\
{$\left[\mathrm{PuClL}_{3}\right]$} & +2.48 & -1.21 & -1.22 & -0.70 & 0.46 & 0.46 & 0.52 \\
\hline
\end{tabular}


Table S14. Summary of values obtained from QTAIM analysis. Charge $q$ and delocalization index $\delta$ for azido substituted compounds $\left[\mathrm{MN}_{3}((S)-\mathrm{PEBA})_{3}\right]$.

\begin{tabular}{llllllll}
\hline & $q(\mathrm{M})$ & $q\left(\mathrm{~N}_{\mathrm{a}}\right)$ & $q\left(\mathrm{~N}_{\mathrm{b}}\right)$ & $q(\mathrm{~N})$ & $\delta\left(\mathrm{M}-\mathrm{N}_{\mathrm{a}}\right)$ & $\delta\left(\mathrm{M}-\mathrm{N}_{\mathrm{b}}\right)$ & $\delta\left(\mathrm{M}-\mathrm{N}_{\mathrm{az}}\right)$ \\
\hline$\left[\mathrm{CeN}_{3} \mathrm{~L}_{3}\right]$ & +2.46 & -1.21 & -1.21 & -0.49 & 0.39 & 0.48 & 0.48 \\
{$\left[\mathrm{ThN}_{3} \mathrm{~L}_{3}\right]$} & +2.79 & -1.23 & -1.24 & -0.55 & 0.36 & 0.42 & 0.46 \\
{$\left[\mathrm{PaN}_{3} \mathrm{~L}_{3}\right]$} & +2.70 & -1.23 & -1.23 & -0.53 & 0.39 & 0.46 & 0.49 \\
{$\left[\mathrm{UN}_{3} \mathrm{~L}_{3}\right]$} & +2.61 & -1.21 & -1.22 & -0.52 & 0.41 & 0.48 & 0.51 \\
{$\left[\mathrm{NpN}_{3} \mathrm{~L}_{3}\right]$} & +2.55 & -1.21 & -1.21 & -0.51 & 0.40 & 0.49 & 0.52 \\
{$\left[\mathrm{PuN}_{3} \mathrm{~L}_{3}\right]$} & +2.49 & -1.21 & -1.22 & -0.48 & 0.45 & 0.46 & 0.49 \\
\hline
\end{tabular}

Table S15. Charges $(q)$ and population analysis based on NBO analysis of tris(amidinate) complexes MX $\left.(\mathrm{L})_{3}\right]$ $\left(\mathrm{M}=\mathrm{Ce}, \mathrm{Th}, \mathrm{Pa}, \mathrm{U}, \mathrm{Np}, \mathrm{Pu} ; \mathrm{X}=\mathrm{Cl}, \mathrm{N}_{3} ; \mathrm{L}=(S)\right.$-PEBA $)$.

\begin{tabular}{lccccc}
\hline & $q(\mathrm{M})$ & $q\left(\mathrm{~N}_{\mathrm{a}}\right)$ & $q\left(\mathrm{~N}_{\mathrm{b}}\right)$ & $q(\mathrm{X})$ & population \\
\hline$\left[\mathrm{CeCl}(\mathrm{L})_{3}\right](\mathbf{4})$ & +1.80 & -0.61 & -0.61 & -0.56 & $6 s^{0.18} 5 d^{1.20} 6 d^{0.04} 4 f^{0.81} 5 f^{0.02}$ \\
\hline$\left[\mathrm{ThCl}(\mathrm{L})_{3}\right](\mathbf{1})$ & +1.80 & -0.65 & -0.68 & -0.44 & $7 s^{0.19} 6 d^{1.09} 7 d^{0.05} 5 f^{0.81} 6 f^{0.10}$ \\
{$\left[\mathrm{PaCl}(\mathrm{L})_{3}\right]$} & +1.65 & -0.61 & -0.63 & -0.44 & $7 s^{0.22} 6 d^{1.11} 7 d^{0.05} 5 f^{1.73} 6 f^{0.28}$ \\
{$\left[\mathrm{UCl}(\mathrm{L})_{3}\right](\mathbf{2})$} & +1.58 & -0.60 & -0.61 & -0.46 & $7 s^{0.22} 6 d^{1.16} 7 d^{0.05} 5 f^{2.81} 6 f^{0.22}$ \\
{$\left[\mathrm{NpCl}(\mathrm{L})_{3}\right](\mathbf{3})$} & +1.61 & -0.60 & -0.61 & -0.49 & $7 s^{0.22} 6 d^{1.13} 7 d^{0.06} 5 f^{3.87} 6 f^{0.15}$ \\
{$\left[\mathrm{PuCl}(\mathrm{L})_{3}\right]$} & +1.72 & -0.59 & -0.64 & -0.56 & $7 s^{0.22} 6 d^{1.05} 7 d^{0.06} 5 f^{4.92} 6 f^{0.06}$ \\
\hline$\left[\mathrm{CeN}_{3}(\mathrm{~L})_{3}\right]$ & +1.86 & -0.61 & -0.61 & -0.53 & $6 s^{0.17} 5 d^{1.16} 6 d^{0.03} 4 f^{0.82} 5 f^{0.02}$ \\
\hdashline$\left[\mathrm{ThN}_{3}(\mathrm{~L})_{3}\right](\mathbf{6})$ & +2.00 & -0.65 & -0.68 & -0.59 & $7 s^{0.18} 6 d^{1.05} 7 d^{0.01} 5 f^{0.71} 6 f^{0.09}$ \\
{$\left[\mathrm{PaN}_{3}(\mathrm{~L})_{3}\right]$} & +1.80 & -0.62 & -0.64 & -0.54 & $7 s^{0.21} 6 d^{1.07} 7 d^{0.01} 5 f^{1.71} 6 f^{0.23}$ \\
{$\left[\mathrm{UN}_{3}(\mathrm{~L})_{3}\right](7)$} & +1.68 & -0.60 & -0.61 & -0.52 & $7 s^{0.20} 6 d^{1.12} 7 d^{0.03} 5 f^{2.82} 6 f^{0.19}$ \\
{$\left[\mathrm{NpN}_{3}(\mathrm{~L})_{3}\right](\mathbf{8})$} & +1.69 & -0.60 & -0.61 & -0.53 & $7 s^{0.21} 6 d^{1.10} 7 d^{0.04} 5 f^{3.89} 6 f^{0.13}$ \\
{$\left[\mathrm{PuN}_{3}(\mathrm{~L})_{3}\right]$} & +1.78 & -0.59 & -0.63 & -0.51 & $7 s^{0.21} 6 d^{1.02} 7 d^{0.04} 5 f^{4.95} 6 f^{0.05}$ \\
\hline
\end{tabular}




\section{Literature}

1. Settineri, N. S.; Garner, M. E.; Arnold, J. A Thorium Chalcogenolate Series Generated by Atom Insertion into Thorium-Carbon Bonds. J. Am. Chem. Soc. 2017, 139 (17), 6261-6269.

2. Rabinovich, E.; Aharonovich, S.; Botoshansky, M.; Eisen, M. S. Thorium 2pyridylamidinates: Synthesis, Structure and Catalytic Activity towards the Cyclooligomerization of $\varepsilon$-caprolactone. Dalton Trans. 2010, 39 (29), 6667-6676.

3. Villiers, C.; Thuéry, P.; Ephritikhine, M. A Comparison of Analogous 4f- and 5fElement Compounds: Syntheses, X-ray Crystal Structures and Catalytic Activity of the Homoleptic Amidinate Complexes $\left[\mathrm{M}\left(\mathrm{MeC}(\mathrm{NCy})_{2}\right)_{3}\right](\mathrm{M}=\mathrm{La}, \mathrm{Nd}$ or U). Eur. J. Inorg. Chem. 2004, 2004 (23), 4624-4632.

4. Settineri, N. S.; Shiau, A. A.; Arnold, J. Two-electron oxidation of a homoleptic U(III) guanidinate complex by diphenyldiazomethane. Chem. Commun. 2018, 54 (77), 1091310916.

5. Wedler, M.; Knösel, F.; Noltemeyer, M.; Edelmann, F. T.; Behrens, U. OrganoactinoidKomplexe: Substituierte Benzamidinat-Anionen als sterische Äquivalente $\mathrm{zu} \eta^{5}-\mathrm{C}_{5} \mathrm{H}_{5}$ und $\eta^{5}-\mathrm{C}_{5} \mathrm{Me}_{5} J$. Organomet. Chem. 1990, 388 (1), 21-45.

6. Dröse, P.; Crozier, A. R.; Lashkari, S.; Gottfriedsen, J.; Blaurock, S.; Hrib, C. G.; Maichle-Mössmer, C.; Schädle, C.; Anwander, R.; Edelmann, F. T. Facile Access to Tetravalent Cerium Compounds: One-Electron Oxidation Using Iodine(III) Reagents. J. Am. Chem. Soc. 2010, 132 (40), 14046-14047.

7. Yin, H.; Carroll, P. J.; Manor, B. C.; Anna, J. M.; Schelter, E. J. Cerium Photosensitizers: Structure-Function Relationships and Applications in Photocatalytic Aryl Coupling Reactions. J. Am. Chem. Soc. 2016, 138 (18), 5984-5993.

8. Yin, H.; Carroll, P. J.; Anna, J. M.; Schelter, E. J. Luminescent Ce(III) Complexes as Stoichiometric and Catalytic Photoreductants for Halogen Atom Abstraction Reactions. J. Am. Chem. Soc. 2015, 137 (29), 9234-9237.

9. Werner, D.; Deacon, G. B.; Junk, P. C.; Anwander, R. Cerium(III/IV) Formamidinate Chemistry, and a Stable Cerium(IV) Diolate. Chem. Eur. J. 2014, 20 (15), 4426-4438.

10. Yang, P.; Zhou, E.; Fang, B.; Hou, G.; Zi, G.; Walter, M. D. Preparation of $\left(\eta^{5}-\mathrm{C}_{5} \mathrm{Me}_{5}\right.$ )$_{2}$ Th(bipy) and Its Reactivity toward Small Molecules. Organometallics 2016, 35 (12), 2129-2139.

11. Zhang, C.; Hou, G.; Zi, G.; Ding, W.; Walter, M. D. A Base-Free Terminal Actinide Phosphinidene Metallocene: Synthesis, Structure, Reactivity, and Computational Studies. J. Am. Chem. Soc. 2018, 140 (43), 14511-14525.

12. Castro-Rodriguez, I.; Nakai, H.; Zakharov, L. N.; Rheingold, A. L.; Meyer, K. A Linear, O-Coordinated $\eta^{1}-\mathrm{CO}_{2}$ Bound to Uranium. Science 2004, 305 (5691), 1757-1759.

13. Lam, O. P.; Franke, S. M.; Nakai, H.; Heinemann, F. W.; Hieringer, W.; Meyer, K. Observation of the Inverse Trans Influence (ITI) in a Uranium(V) Imide Coordination Complex: An Experimental Study and Theoretical Evaluation. Inorg. Chem. 2012, 51 (11), 6190-6199. 
14. Wildman, E. P.; Ostrowski, J. P. A.; King, D. M.; Lewis, W.; Liddle, S. T. Uraniumhalide and -azide derivatives of the sterically demanding triamidoamine ligand TrenTPS [TrenTPS $\left.=\left\{\mathrm{N}\left(\mathrm{CH}_{2} \mathrm{CH}_{2} \mathrm{NSiPh}_{3}\right)_{3}\right\}_{3}{ }^{-}\right]$. Polyhedron 2017, 125, 2-8.

15. King, D. M.; Tuna, F.; McInnes, E. J. L.; McMaster, J.; Lewis, W.; Blake, A. J.; Liddle, $\mathrm{S}$. T. Isolation and characterization of a uranium(VI)-nitride triple bond. Nature Chem. 2013, 5, 482-488.

16. Thomson, R. K.; Cantat, T.; Scott, B. L.; Morris, D. E.; Batista, E. R.; Kiplinger, J. L. Uranium azide photolysis results in $\mathrm{C}-\mathrm{H}$ bond activation and provides evidence for a terminal uranium nitride. Nature Chem. 2010, 2 (9), 723-729.

17. Evans, W. J.; Montalvo, E.; Ziller, J. W.; DiPasquale, A. G.; Rheingold, A. L. Uranium Metallocene Complexes of the 1,3,4,6,7,8-Hexahydro- $2 \mathrm{H}$-pyrimido[1,2-a]pyrimidinato Ligand, (hpp) ${ }^{-}$. Inorg. Chem. 2010, 49 (1), 222-228.

18. Fichter, S.; Kaufmann, S.; Kaden, P.; Brunner, T. S.; Stumpf, T.; Roesky, P. W.; März, J. Enantiomerically pure Tetravalent Neptunium Amidinates: Synthesis and Characterization. Chem. Eur. J. 2020, 26 (41), 8867-8870.

19. Kirishima, A.; Kimura, T.; Nagaishi, R.; Tochiyama, O. Luminescence properties of tetravalent uranium in aqueous solution. Radiochim. Acta 2004, 92 (9-11), 705-710.

20. Komyak, A. I.; Zazhogin, A. P.; Umreiko, D. S.; Lugovsky, A. A. Electronic spectra of uranium tetrachloride clusters with electron-donor ligands. J. Appl. Spectrosc. 2009, 76 (2), 167-173.

21. Aoyagi, N.; Watanabe, M.; Kirishima, A.; Sato, N.; Kimura, T. Optical Properties of Tetravalent Uranium Complexes in Non-Aqueous Media. J. Radioanal. Nucl. Chem. 2015, 303 (2), 1095-1098.

22. Schneider, D.; Spallek, T.; Maichle-Mössmer, C.; Törnroos, K. W.; Anwander, R. Cerium tetrakis(diisopropylamide) - a useful precursor for cerium(iv) chemistry. Chem. Commun. 2014, 50 (94), 14763-14766.

23. Williams, U. J.; Carroll, P. J.; Schelter, E. J. Synthesis and Analysis of a Family of Cerium(IV) Halide and Pseudohalide Compounds. Inorg. Chem. 2014, 53 (12), 63386345 .

24. Rice, N. T.; Su, J.; Gompa, T. P.; Russo, D. R.; Telser, J.; Palatinus, L.; Bacsa, J.; Yang, P.; Batista, E. R.; La Pierre, H. S. Homoleptic Imidophosphorane Stabilization of Tetravalent Cerium. Inorg. Chem. 2019, 58 (8), 5289-5304. 